\title{
WestVirginiaUniversity
}

THE RESEARCH REPOSITORY @ WVU

Graduate Theses, Dissertations, and Problem Reports

2006

\section{Measuring chemotaxis in Borrelia burgdorferi the Lyme disease spirochete}

Richard Gerrit Bakker

West Virginia University

Follow this and additional works at: https://researchrepository.wvu.edu/etd

\section{Recommended Citation}

Bakker, Richard Gerrit, "Measuring chemotaxis in Borrelia burgdorferi the Lyme disease spirochete" (2006). Graduate Theses, Dissertations, and Problem Reports. 2423.

https://researchrepository.wvu.edu/etd/2423

This Dissertation is protected by copyright and/or related rights. It has been brought to you by the The Research Repository @ WVU with permission from the rights-holder(s). You are free to use this Dissertation in any way that is permitted by the copyright and related rights legislation that applies to your use. For other uses you must obtain permission from the rights-holder(s) directly, unless additional rights are indicated by a Creative Commons license in the record and/ or on the work itself. This Dissertation has been accepted for inclusion in WVU Graduate Theses, Dissertations, and Problem Reports collection by an authorized administrator of The Research Repository @ WVU.

For more information, please contact researchrepository@mail.wvu.edu. 
Measuring Chemotaxis in Borrelia burgdorferi the Lyme Disease Spirochete

\author{
Richard Gerrit Bakker
}

Dissertation submitted to the School of Medicine

at West Virginia University

in partial fulfillment of the requirements for the degree of

Doctor of Philosophy

In

Microbiology and Immunology

\author{
Nyles W. Charon Ph.D. (Chair) \\ Melanie Fisher MD \\ Daniel Flynn Ph.D. \\ Michael Miller Ph.D. \\ Rosana Schafer Ph.D. \\ David Yelton Ph.D.
}

Department of Microbiology Immunology and Cell Biology

\author{
Morgantown, West Virginia \\ 2004
}

Keywords: Borrelia burgdorferi, chemotaxis, flow cytometry, Lyme disease, CheA complementation

Copyright 2004 Richard G. Bakker 


\begin{abstract}
Measuring Chemotaxis in Borrelia burgdorferi the Lyme Disease Spriochete Richard Gerrit Bakker
\end{abstract}

Borrelia burgdorferi is the spirochete causative agent of Lyme disease. The chemotaxis and motility systems of these bacteria are far less well described than that of Escherichia coli or Salmonella enterica. This dissertation explores the role of the CheA proteins in the chemotactic response and describes the first defined attractants for $B$. burgdorferi.

In order to test hypotheses, we developed or optimized three protocols. To characterize the motion of cells, two motion tracking systems were optimized. The Hobson BacTracker allowed for tracking cell motions in real time. This hardware/software chimera, while powerful for the specific application, utilizes a cumbersome interface. Therefore, the software package Volocity was adopted. While the tracking itself is somewhat slower, the interface greatly facilitates data collection, organization, and presentation, making it much faster. To assay chemotaxis with the capillary tube assay, one must enumerate cells. This was previously difficult because cell enumeration was slow, laborious, and ineffectual at low concentrations. We overcame these limitations by initially developing a protocol for enumerating cells by flow cytometry. Once this enumeration method was validated with direct comparisons to Petroff-Hausser counting chamber data, we were able to screen for attractants using a modified capillary tube assay.

We found that $B$. burgdorferi mutants in che $A 2$ were non-chemotactic to defined attractants. Complementation of cheA2 restored the wild-type phenotype. Mutants in cheA1 failed to show any discernable phenotype. The modified capillary tube chemotaxis assay was used to screen for chemoattractants. To date serine, glycine, $\mathrm{N}$-n-diacetyl-chitobiose, glucose, glutamate, putricine, spermidine, rabbit serum, and glucosamine, have been tested, the latter five had 
at least some chemoattractant ability. Finally, this is the first work to correlate the ability of the cells to reverse with chemotaxis.

In conclusion, this work developed techniques to track the motion of $B$. burgdorferi and measure the chemotactic response with a high throughput assay. These tools are being used in a screen of compounds which has already found 5 specific compounds that act as chemoattractants. The techniques developed will be useful not only for $B$. burgdorferi, but will facilitate measuring the chemotactic response in other slow growing prokaryotic species. 
I wish to dedicate this work to:

\author{
My Parents: \\ Leslie and Geert Bakker
}

and

My Grandmother:

Ellen Smith

Thank you for all your support, without you none of this would have been possible. 
I wish to acknowledge and thank a number of people for their time, help, and support over the course of my research.

At West Virginia University:

The Charon laboratory - for all the help and patience

Dr. Nyles Charon

Melanie Sal

Dr. Chunhao Li

Dr. Md. Motaleb

Pang Jia

My fellow graduate students - for technical help and harassment

Lisa Rucks

Matt Hirsh

Jeff Miller

Alicia Mathers

Sarah Dodson

The Flow Cytometric Core Facility

Dr. Cynthia Cunningham - for help, technical and otherwise, as well as chocolate provisions

At the University of California Los Angles:

Dr. Renata Lux - for teaching me tissue penetration assays

At the University of Minnesota:

Dr. Stuart G. Goldstein - for making mathematics work for me

My Committee:

Melanie Fisher MD

Daniel Flynn Ph.D.

Michael Miller Ph.D.

Rosana Schafer Ph.D.

David Yelton Ph.D.

At home:

Mark A. Blake - for being you, and putting up with me 
Table of Contents

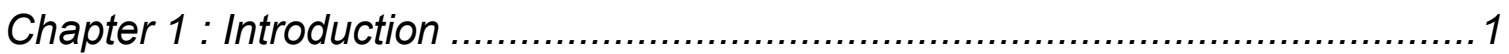

A. History of Lyme Disease and Classification of Borrelia species ..................... 2

B. Structure and Motility of B. burgdorferi and Escherichia coli. ......................... 4

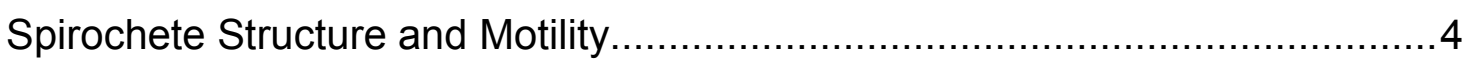

Escherichia coli Motility and Chemotaxis .......................................................

Similarities and Differences between E. coli and B. burgdorferi ..................... 9

C. Clinical manifestations of Lyme Borreliosis ............................................ 10

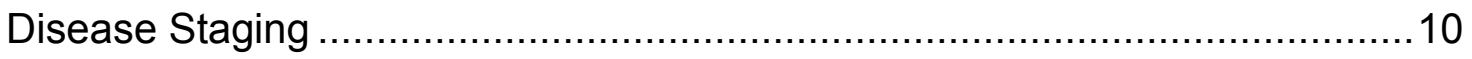

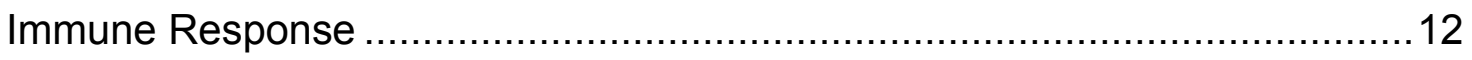

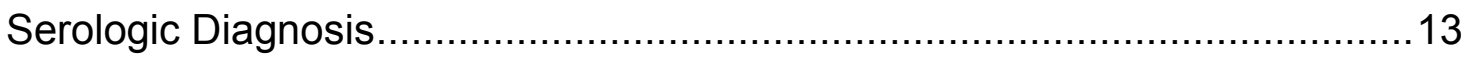

European and Asian Manifestations of Disease ......................................... 14

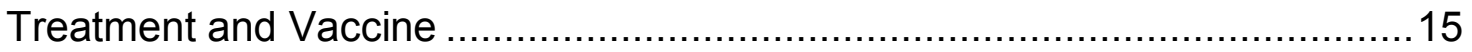

D. Chemotaxis and Motility as Virulence Factors........................................ 16

Chemotaxis and Motility as Virulence Factors in B. burgdorferi ..................... 17

Chemotaxis and Motility as Virulence Factors in Other Species ...................17

Chemotaxis and Motility as Virulence Factors in other Spirochete Species ....19

E. Borrelia burgdorferi Life Cycle and Gene Expression................................20

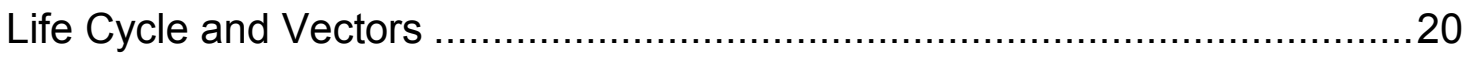

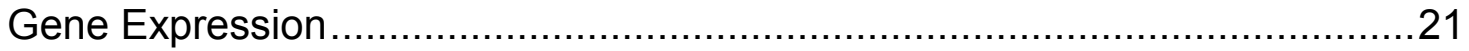

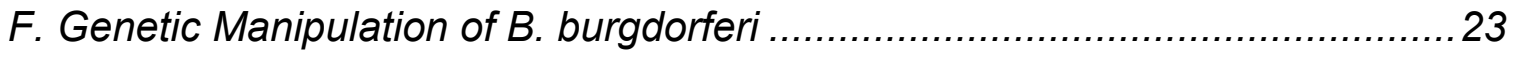

Chromosome Structure and Replication .................................................24

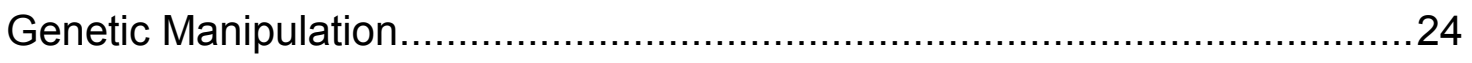

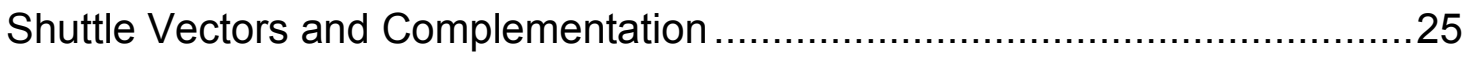

Lateral Gene Transfer: Transposons and Phages....................................25

G. The Structure and Function of Methyl Accepting Chemotaxis Proteins.........26

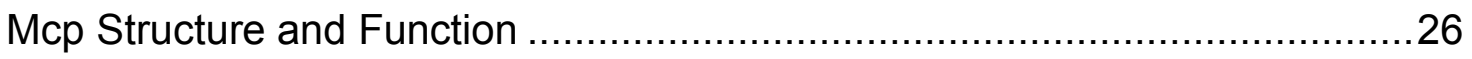

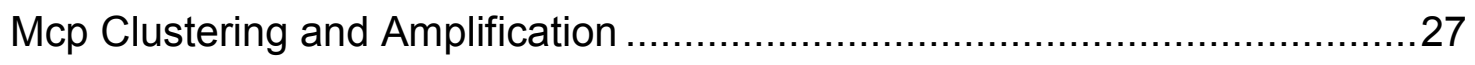

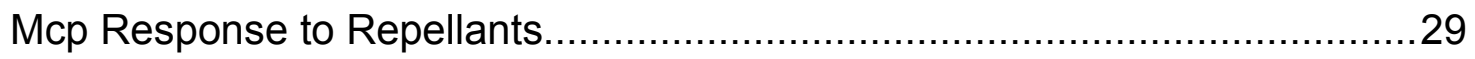

$H$. The Structure and Function of Chemotaxis Protein A (CheA) ....................... 30

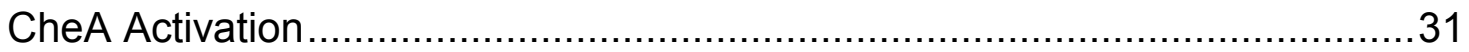

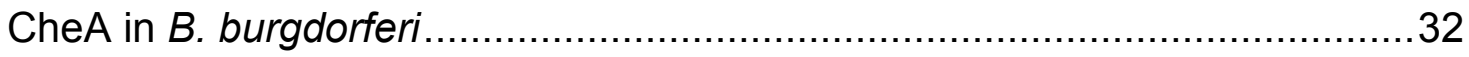


I. Capillary Tube Assay and Flow Cytometric Enumeration of B. burgdorferi......33

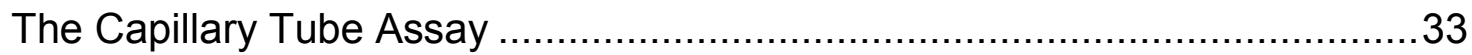

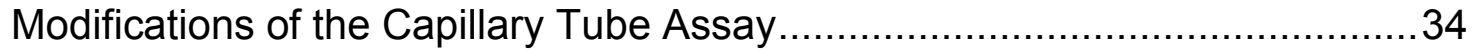

Enumeration of $B$. burgdorferi using Flow cytometry................................. 35

Flow Cytometric Enumeration of $B$. burgdorferi............................................ 36

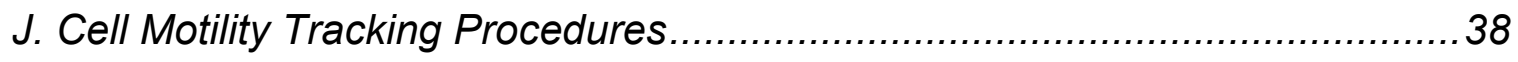

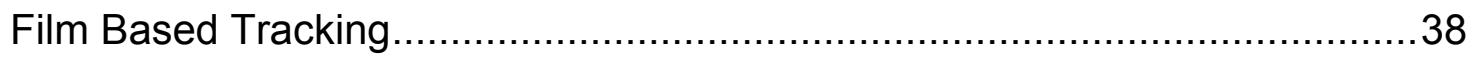

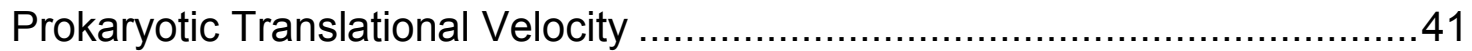

Chapter 2: Asymmetrical flagellar rotation in Borrelia burgdorferi cheA mutants 43

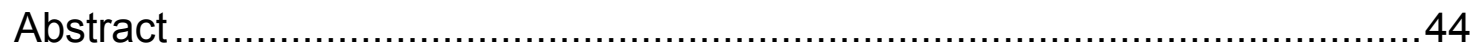

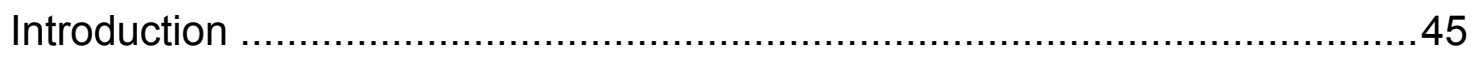

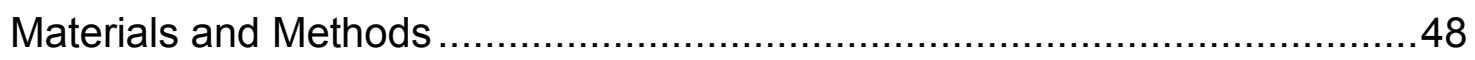

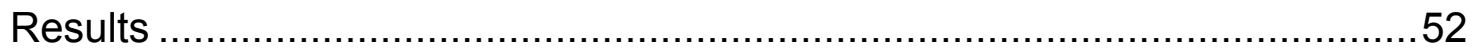

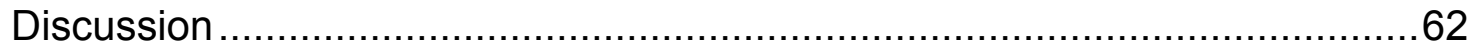

Chapter 3: Identification of specific chemoattractants for Borrelia burgdorferi: A

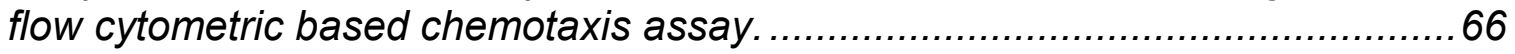

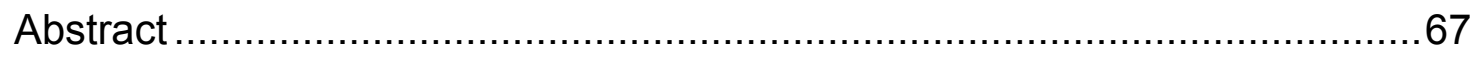

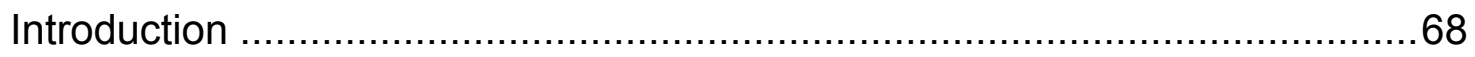

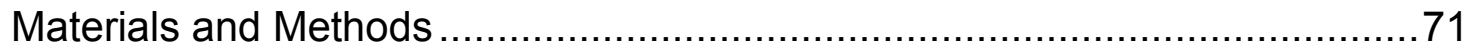

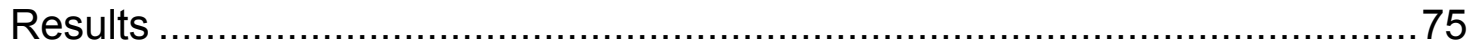

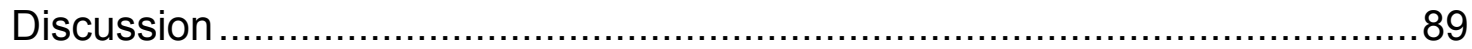

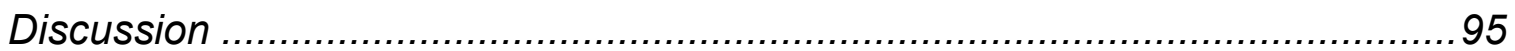

Identification of specific chemoattractants for Borrelia burgdorferi: A flow

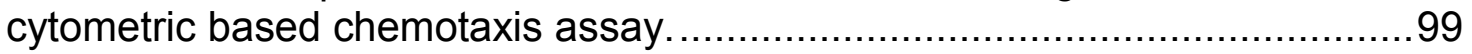

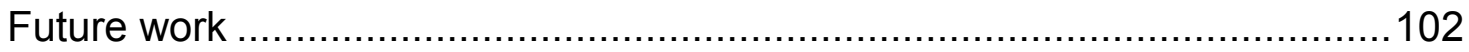

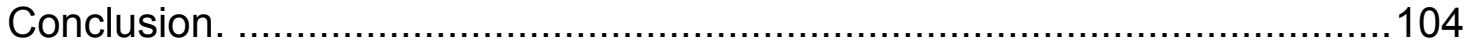

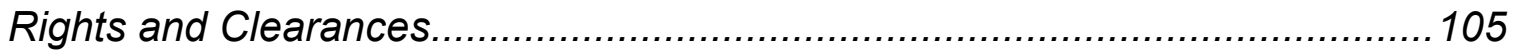

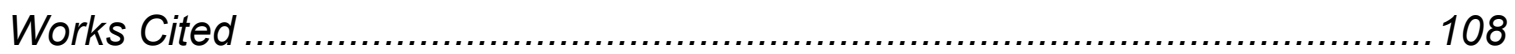

Curriculum Vitae: Richard Gerrit Bakker ................................................ 137 


\section{Table of Figures}

Figure 1.1: $B$. burgdorferi longitudinally and in cross section......................... 4

Figure 1.2: Possible models of swimming $B$. burgdorferi ..................................

Figure 1.3: The signal cascade controlling direction of rotation in E. coli.............7

Figure 1.4: Biased Random Walk. Two idealized E. coli translational trajectories

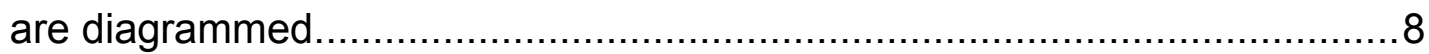

Figure 1.5: A swimming track of $B$. burgdorferi generated with the tracking

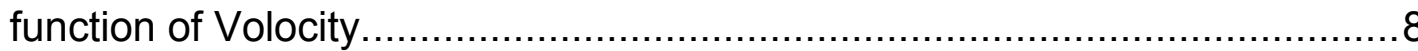

Figure 2.1: Swimming cells of $B$. burgdorferi as a function of direction of rotation of the periplasmic flagella. 46

Figure 2.2: Genetic map of the flaA and (b) cheW2 clusters. Wide arrows indicate direction of transcription

Figure 2.3 (a) Primer extension results of cheW2 cluster show a $\sigma^{70}$-like recognition sequence.

Figure 2.4 Western blot analysis using different antisera reacted against whole cell lysates of Wild Type and cheA mutants.

Figure 2.5 RT-PCR results indicating that transcription initiating within kan allows for downstream transcription of truncated cheA2 and cheW3. 56

Figure 2.6: (a) Capillary tube (b), and swarm plate assays for chemotaxis of wildtype and mutants. 58

Figure 2.7: Sequential video frames of wild-type and LC-A1A2 mutant taken every 0.33 seconds 61

Figure 3.1 Four representative dot plots with identical gating. 76

Figure 3.2: Flow cytometry (FACS) vs. Petroff Hausser Counting. 77

Figure 3.3 The chemotactic response as a function of the number of cells in the cell pool. 79

Figure 3.4: Chemotactic response per assay time. 80

Figure 3.5: $U$ Tube vs. $2 \mathrm{ml}$ microfuge tube assay for measuring chemotaxis....81

Figure 3.6: Effect of increasing assay viscosity. .82

Figure 3.7: The chemotactic response of cells in preparations of motility buffer made with different BSA formulations. 
Figure 3.8: The chemotactic response as a function of culture age.

Figure 3.9: The chemotactic response of cells starved in motility buffer before beginning the assay.

Figure 3. 10: The Response Profile of Wild-type (B31), LC-A2 and cheA2 complemented

Figure 3.11: Estimation of the Attractant Gradient.

\section{Table of Tables}

Table 1.1: A summary of published translational velocities for selected prokaryotes.

Table 2.1 Plasmids and sequences of primers. 49

Table 2.2 Reversal Frequency and Translational Velocity.

Table 3.1 Representative FACScalibur Machine Settings.

Table 3.2: Average response of $B$. burgdorferi strain B31A to different chemoattractants 86

Table 3.3: Relative Response to screened chemoattractants in the capillary tube assay. 87

Table 3.4: Translational velocities one standard deviation .89

\section{Abbreviations / Acronyms}

ACA Acrodermatitis chromium atrophicans

CCW Counter clockwise

CFU Colony forming unit

CheB Chitosan Dimers

CheA Chemotaxis protein $A$

CheB Chemotaxis protein B

CheW Chemotaxis protein $W$

CheY Chemotaxis protein $\mathrm{Y}$

CW Clockwise 


$\begin{array}{ll}\text { EDTA } & \text { Ethylenediamine-tetraacetic acid } \\ \text { EM } & \text { Erythemia migrans } \\ \text { FACS } & \text { Fluorescent activated cell sorting } \\ \text { GFP } & \text { Green fluorescent protein } \\ \text { Glc } & \text { Glucosamine } \\ \text { Inf- } \gamma & \text { Interferon Gamma } \\ \text { Mcp } & \text { Methyl accepting chemotaxis protein } \\ \text { NaG } & \text { N-acetyl-glucosamine } \\ \text { OspA } & \text { Outer surface protein A } \\ \text { OspB } & \text { Outer surface protein B } \\ \text { OspD } & \text { Outer surface protein D } \\ \text { OspE } & \text { Outer surface protein E } \\ \text { PCR } & \text { Polymerase chain reaction } \\ \text { PF's } & \text { Periplasmic flagella } \\ \text { PMN } & \text { Polymorphic neutrophil } \\ \text { TLR } & \text { Toll like receptor }\end{array}$




\section{Chapter 1: Introduction}

Motility was the genesis of microbiology. Antonie van Leeuwenhoek first observed swimming bacteria in water samples in the late 1600's. It is doubtful Leeuwenhoek would have realized that they were alive had they not swam in a purposeful fashion (10). Borrelia burgdorferi is the spirochete that causes Lyme disease, the most prevalent vector borne disease in the United States (249). The long-term aim of our work is to relate chemotaxis and motility to the pathogenesis of $B$. burgdorferi. This dissertation focuses on measuring and understanding the chemotactic response of $B$. burgdorferi.

As a primer to presentation of data in Chapters 2 and 3, the Introduction Section will briefly review the literature in relation to:

a. The history of Lyme disease and classification of Borrelia species.

b. The structure and motility of B. burgdorferi and Escherichia coli.

c. The clinical manifestations of Lyme disease.

d. Chemotaxis and motility as bacterial virulence factors.

e. The $B$. burgdorferi life cycle and gene expression profiles.

f. Genetic manipulation of $B$. burgdorferi.

g. The structure and function of methyl accepting chemotaxis proteins (Mcps).

h. The structure and function of chemotaxis protein $A(C h e A)$.

i. The capillary tube assay and flow cytometric enumeration of $B$. burgdorferi.

j. Cell motility tracking procedures.

Section headings are bold face and underlined while subsection headings are found in bold. Following the Introduction, two manuscripts containing published or in preparation work are included, concluding with a discussion. 


\section{A. History of Lyme Disease and Classification of Borrelia species}

Borrelia burgdorferi is the causative agent of Lyme disease. The disease is named for Old Lyme Connecticut where it caused a cluster of apparent juvenile rheumatoid arthritis beginning in 1972. The arthritis was geographically and temporally clustered in residents of Old Lyme and surrounding communities in the summer season. Dr. Alan Steere, M.D., of Yale University, first studied the outbreak of unusual symptoms $(253,255,256)$. A migratory rash that sometimes exhibited central clearing often preceded symptoms. Concomitant with this peculiar rash, headache, stiff neck, fever, myalgias, arthralgias, malaise, fatigue, and / or lymphadenopathy were often observed. Later, meningoencephalitis, cranial or peripheral neuropathies, myocarditis, cranial or peripheral neuropathies, or atrioventricular-node block could occur. After experiencing these symptoms, patients could relapse and/or develop arthritis. It was noted that penicillin or tetracycline shortened the duration of the skin lesions and often prevented the subsequent symptomatology $(253,256)$.

The spirochetal etiology of these symptoms, as well as the Ixodes dammini arthropod transmission vector, was postulated early in the outbreak. Patients developed a delayed immune response to the organism and cells with typical spirochetal morphology were recovered from skin biopsies (253). In 1981 while surveying for rickettsia in ticks captured on Shelter Island, New York, Dr. Willy Burgdorfer, of the Rocky Mountain Laboratories section of the National Institutes of Health, identified and described a species of spirochete found in tick guts (Ixodes dammini, now I. scapularis). The localization of spirochetes in an arthropod vector from an endemic area for Lyme disease raised a high index of suspicion $(22,38,253,255,256)$. Subsequently, the spirochete was classified as a Borrelia species and named for Dr. Burgdorfer (117). 
In 1883, Alfred Buchwald in Germany first published a description of skin lesions similar to those described by Steere. Arvid Afzelius reported similar lesions at a 1909 meeting in Stockholm and his suspicion the lesions were caused by a tick bite (190). While these lesions were described, no anti-microbial therapy was available. These lesions were likely the classic erythema migrans lesions or acrodermatitis chronica atrophicans lesions which are discussed in the section on medical implications of Lyme borreliosis.

\section{Classification of Borrelia burgdorferi.}

Spirochetes are one of 40 major bacterial phyla and the only one that can be determined by microscopy alone. They are separated into 9 genera: Borrelia, Brevinema, Brachyspira, Cristispira, Leptospira, Leptomena, Spirocheata, Spironema and Treponema (197). All are helical or wave shaped organisms with periplasmic flagella used for locomotion. These flagella are sandwiched between an outer membrane sheath and the cell cylinder. They extend down the cylinder, and depending on the species may overlap in the center. Almost all known Borrelia are thought to be pathogens or parasites, host associated, and microaerophilic $(89,91,197)$. However, all of the above characteristics are not documented for every Borrelia.

A number of pathogenic Borrelia family members cause Lyme borreliosis symptoms. This family is known as Borrelia burgdorferi sensu lato. The members of this family to date are Borrelia burgdorferi sensu stricto, $B$. garinii, $B$. afzelii, B. japonica, B. valaisiana, B. lusitaniae, B. bissetti, $B$, andersonii, $B$. tanukii, B. turdi, and B. sincia (41, 165, 284, 285 and references within). While all are thought to be parasitic to mammals, only B. burgdorferi s.s., B. garinii, $B$. afzelii $(285)$, B. Iusitaniae $(55,138)$, and B. bissettii $(185,202,208)$ have been shown to be pathogens in humans. B. valaisiana is likely pathogenic as DNA from this species has been PCR amplified from patients $(41,165)$. The symptomatology caused by this group is not uniform. This diversity leads to difficulty in diagnosis and proper treatment. 


\section{B. Structure and Motility of B. burgdorferi and Escherichia coli.}

\section{Spirochete Structure and Motility}

Spirochetes are Gram-negative bacteria with a peptidoglycan layer on the surface of a protoplasmic cell cylinder. This cylinder lies within an outer membrane sheath. Sandwiched between the cell cylinder and the sheath are the flagella, earning them the name periplasmic flagella (PFs) (Figure 1.1) $(91,107)$. While the structures of spirochetes are similar, they translate either in a helical manner or a flat wave depending on species $(49,50,89,125,229,281)$. This method of locomotion is ideal for translation in viscous gel-like media $(25,131$, $201,218)$. Spirochete motility is postulated to be an important virulence factor (118). Because spirochetes are generally fastidious, slow growing organisms, with many genetic tools still new or in development, the description of their motility and chemotaxis apparatuses has lagged behind other species (118 and references within).

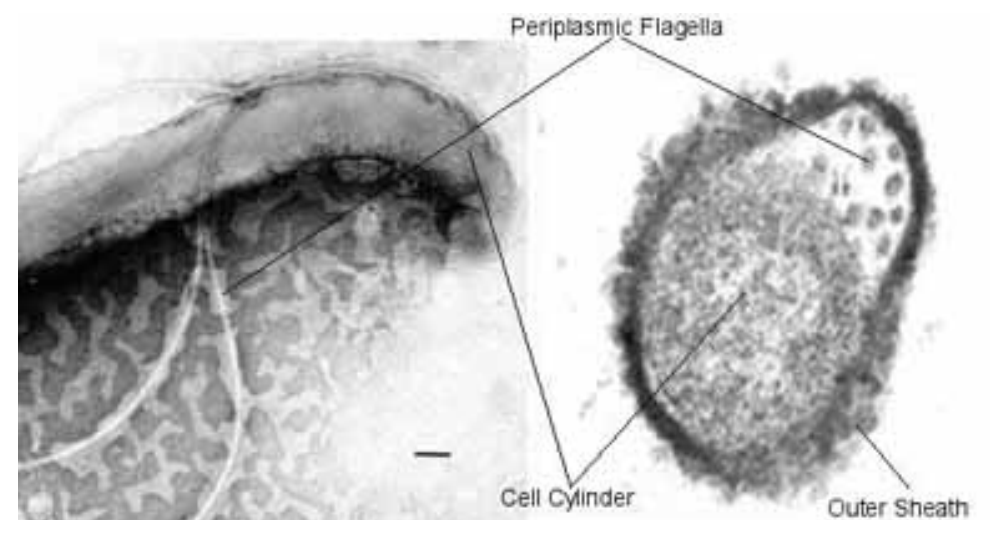

Figure 1.1: $B$. burgdorferi longitudinally and in cross section. The bar in the lower right hand corner of the micrograph on the left is $0.1 \mu \mathrm{m}$ long, and is valid only for that micrograph.

B. burgdorferi translates (swims) as a flat wave (sine wave on the $X Y$ axis) using bundles of 7 to 11 PFs inserted subterminally at each cell pole (91). Aflagellar mutants are rod-shaped and non-motile indicating that flagella play a structural as well as functional role $(176,219)$. The following model seeks to explain the generation of the backward propagating flat wave that leads to cell translation. The model is based on three observations and two definitions: 1) mutants without PFs are rod shaped, 2) isolated PFs are left-handed, and 3) the helix pitch of the 
PFs is equivalent to the wavelength of the cell. A left-handed helix is defined as a helix that moves in a counter-clockwise (CCW) direction going away from the observer, whereas a right-handed helix moves in a clockwise (CW) direction. Direction of motor rotation is defined by looking along the PF toward the flagellar motor. Goldstein et. al. (1994) proposed a flexible rod (cell cylinder) wrapped in a right-handed fashion, around a left-handed helix (flagella), would result in a flat wave. The model states that rotations of the PFs must be coordinated for translation to occur. When PFs rotate in the same direction, the waves generated at the poles are out of phase. When out of phase waves collide, the cell cylinder often bends. Bending actions, which are non-translational, are known as flexes $(89,91,144)$. In order for translation to take place, the motors at each cell pole must rotate in opposite directions as is demonstrated in Figure 1.2. A discussion of the possible roles for the chemotaxis system in this asymmetry is contained in the discussion of CheA.

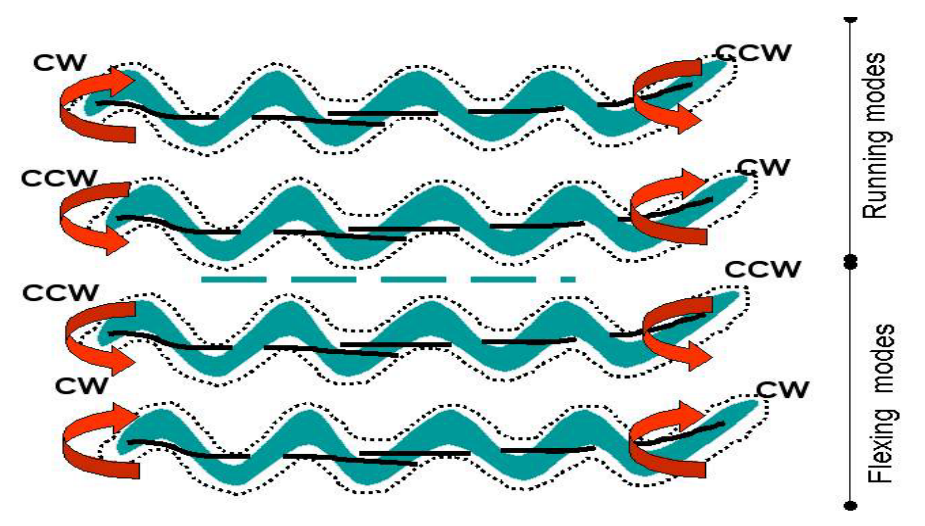

Figure 1.2: Possible models of swimming $B$. burgdorferi.

The arrows and the abbreviations at the end of the cell indicate the directions the motors are running. The top two models would result in translation and are labeled "Running modes". The two models on the bottom would result in the cell bending and are labeled "Flexing modes". This diagram assumes that all motors run at the same speed.

\section{Escherichia coli Motility and Chemotaxis}

Escherichia coli running is correlated with CCW rotation of the flagella, and tumbling is associated with CW rotation. E. coli chemotaxis and motility are discussed as a classic model and illustrated in Figure 1.3. In the molecular description of chemotaxis, a "memory" characteristic was noted. It appeared that if an E. coli cell was stimulated by an attractant, it eventually stopped responding 
to this attractant and could not be re-stimulated for several seconds $(35,159)$. Additionally this chemotactic response was found to be dependant upon methionine (134). It was then noted that proteins in the membrane fraction underwent methylation following cell exposure to attractants $(59,122,134)$. These methylated proteins became known as methyl accepting chemotaxis proteins (Mcp) and in large part control the chemotactic abilities of the cell. The Mcps are integral membrane proteins, which exist in a complex with the internal chemotaxis proteins CheA and CheW. If an attractant is bound to the extracytoplasmic domain of the Mcp, there is a conformational change that increases the probability the cytoplasmic domain will be methylated. S-adenosylL-methionine donates the methyl group through the enzymatic action of CheR. There are eight conserved glutamate residues on the cytoplasmic domain of the Mcp where methylation takes place (30). As these eight sites are filled, tumbling is suppressed by reduced auto-phosphorylation of CheA. Once the cytoplasmic tails of the Mcp are fully methylated, CheA auto-phosphorylation is no longer suppressed and the probability of tumbling is increased. The process of suppressing tumbling activity by squelching CheA autophosphorylation is known as excitation $(7,247)$. The process of turning off suppression of CheA autophosphorylation is known as adaptation (11). CheB is a methylesterase that demethylates the cytoplasmic tails of the Mcp, to return them to a ground state. CheB activity is increased approximately 10 times by phosphorylation via CheA in a reaction that occurs more slowly than CheA phosphorylating CheY $(32,195)$. CheA autophosphorylation provides the energy to signal a motor direction switch. Phosphorylated CheA passes the phosphoryl group onto the response regulator $\mathrm{CheY}$. Phosphorylated $\mathrm{CheY}$ interacts with the motor switch protein, FliM, to increase the probably of $\mathrm{CW}$ rotation which will lead to tumbling in E. coli. CheZ de-phosphorylates CheY decreasing the affinity of CheY for FliM $(8,10,24,31,134,137,152,244)$. Detailed descriptions of CheA and the Mcps are found later in the Introduction. 


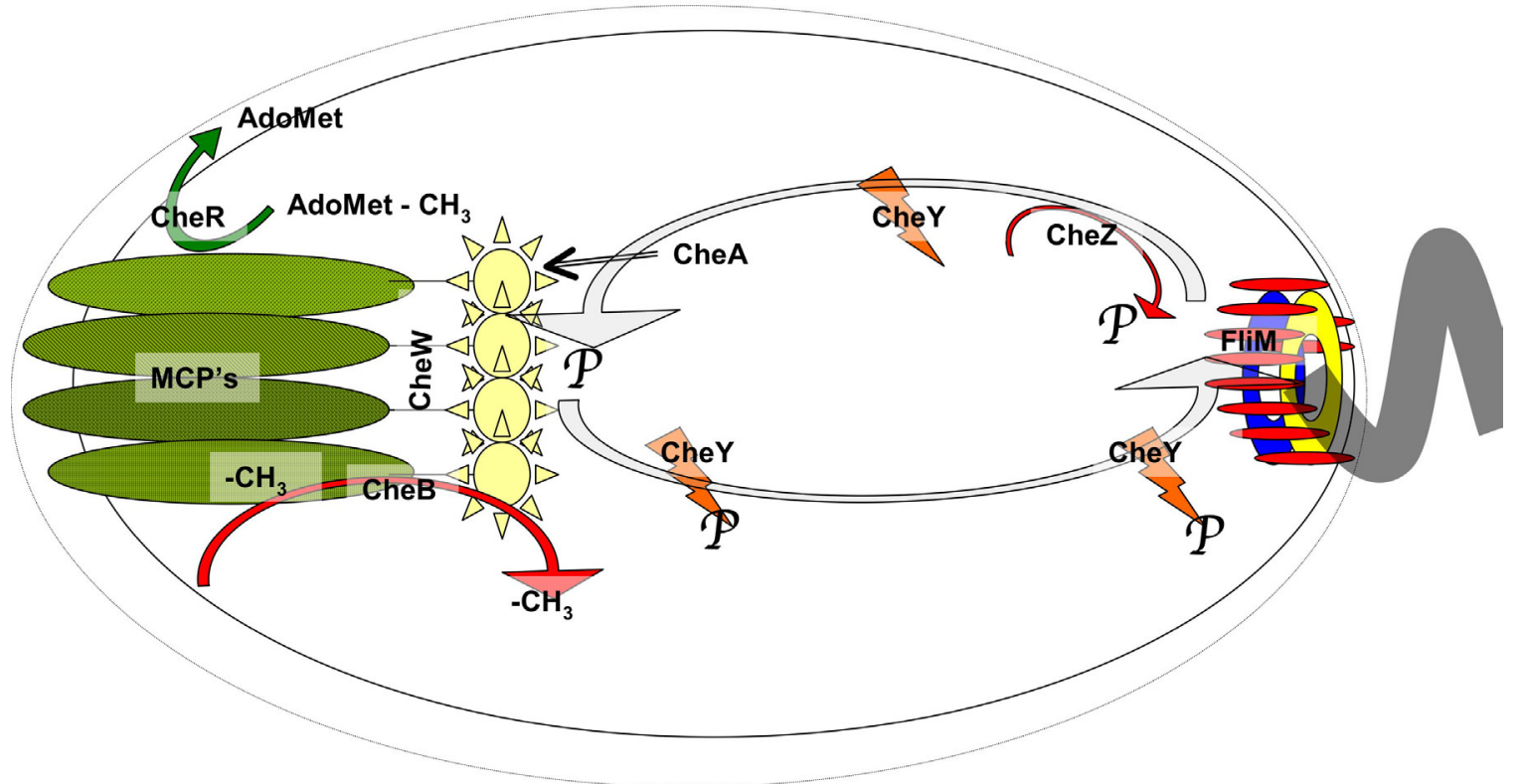

Figure 1.3: The signal cascade controlling direction of rotation in E. coli. Che is the designation for chemotaxis protein and MCP is the designation for Methyl Accepting Chemotaxis Protein. The CheA (starburst) autophosphorylation activity allows it to phosphorylate $\mathrm{CheY}$ (lighting bolt). CheY phosphate then interacts with the flagellar motor protein FliM protein to destabilize counter-clockwise rotation, leading to clockwise rotation. Binding of chemoattractants to the Mcps in the periplasmic space leads to methylation of the cytoplasmic portion of the molecule by the action of CheR. Demethylation occurs much more slowly and is catalyzed by CheB. Binding of attractants to Mcps occurs concomitantly with a reduction in CheA autophosphorylation activity. Adapted from (32).

This molecular switch mechanism leads to a translational function up a gradient known as a biased random walk (23). Because $E$. coli cannot turn toward an attractant it must re-orient itself in a tumble. While this reorientation is nearly random, cells with fewer than four flagella are more likely to make smaller directional changes between runs, and buffeting by Brownian motion can effect the direction of swimming (280). Upon reorientation the cell swims in a vector pattern (Figure 1.4) and temporally samples attractant concentration in the new location. Thus, $E$. coli uses a temporal gradient when undergoing chemotaxis. By increasing run length, cells translate farther from origin in a given time even if the directional changes are the same. This is illustrated in Figure 1.4 (adapted 
from $(10,23))$. This mode of translation contrasts to $B$. burgdorferi that is unable to tumble due to cell length. B. burgdorferi can flex and reverse, sometimes reorienting itself, but it is also able to translate in a curved pattern (Figure 1.5). B. burgdorferi modes of translation are demonstrated in Figure 1.2.

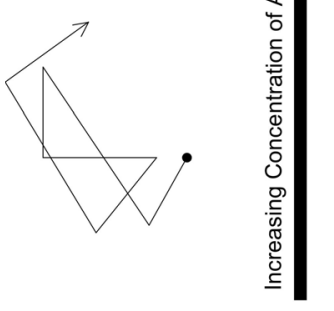

A

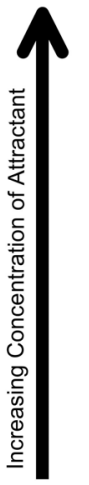

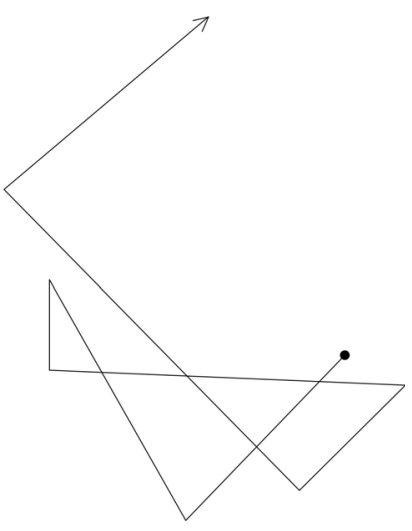

B
Figure 1.4: Biased Random Walk. Two idealized E. coli translational trajectories are diagrammed.

(A) characterizes movements in the absence of an attractant. (B) characterizes movements in the presence of an attractant. If runs become longer due to Mcp mediated attractant suppression of CheA activity, the direction of cell reorientation does not affect how

far the cell will move from the origin. Adapted from $(10,23)$.

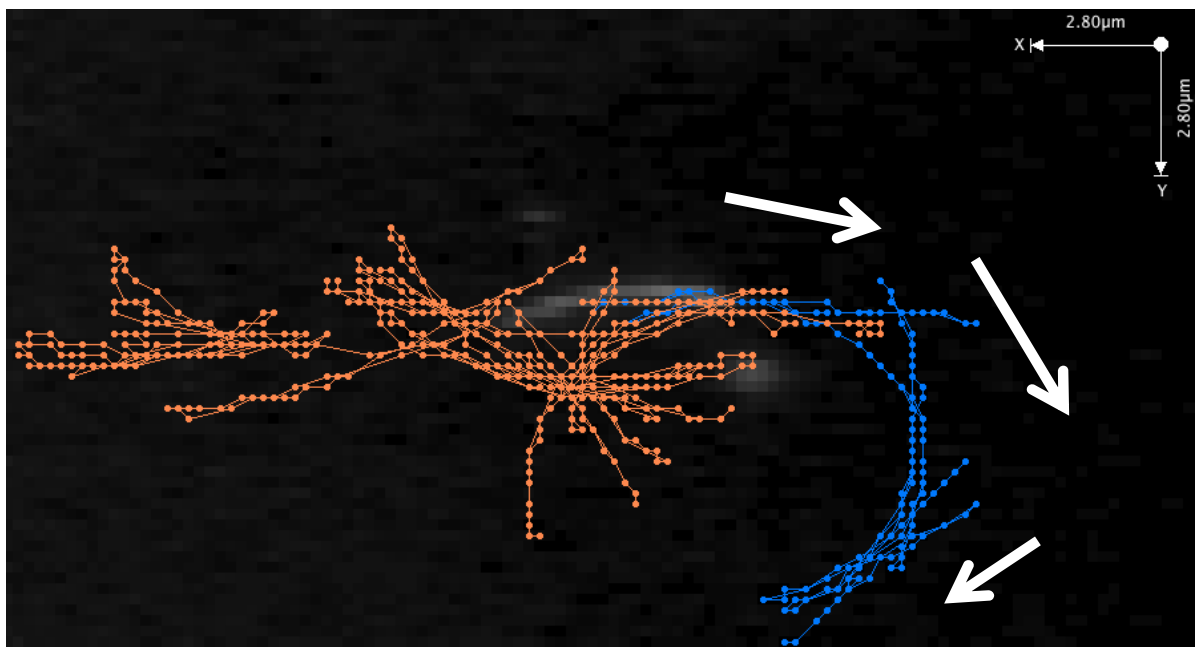

Figure 1.5: A swimming track of $B$. burgdorferi generated with the tracking function of Volocity. A swimming track of $B$. burgdorferi generated

with the tracking function of Volocity. Each dot represents the location of the center of the cell in each frame of the video. The tracks demonstrate B. burgdorferi is able to translate in curves (arrows). E. coli predominately translates in straight lines (figure 1.9). The tracked cell swam for 147.5 seconds and reversed 25 times. Two tracks were generated when the cell went out of focus and then returned to the same focal plain. Returning to the focal plane caused the cell to be read as a new object. See Introduction section $\mathrm{J}$ for an explanation of how this figure was generated. 


\section{Similarities and Differences between E. coli and B. burgdorferi}

There are contrasts between the motility and chemotaxis apparatuses in $E$. coli and $B$. burgdorferi. B. burgdorferi has multiple copies of several chemotaxis and motility genes that are constitutively expressed under the control of $\sigma^{70}$ like promoters $(79,103,146)$. In contrast, $E$. coli have most motility genes under cascade control of $\sigma^{28}$-like promoters (120). The exceptions are the early motility genes, flhC and $f l h D$ that are expressed under the control of several regulatory signals including catabolite repression (6 and references within). Expression of these genes then allows for expression of downstream elements (52). Given these differences, it is significant that the phenotypes of mutants in genes having high identity are often the same between the species, suggesting an overall conservation of function $(40,47)$.

How $B$. burgdorferi changes direction is not entirely understood. After flexing, the cells are sometimes reoriented in space so it seems that this translational mode takes the place of the E. coli tumble. However, tracking data shows that a long straight swimming track is the exception rather than the rule for $B$. burgdorferi in the absence of an attractant (Figure 1.5). Mathematical modeling and swimming patterns of specific species have suggested that spatial sensing of chemoattractants is possible $(65,269)$. Spatial sensing would require two sensor patches, at opposite cell-poles. Theoretically, cells as short as $1 \mu \mathrm{m}$ could use this mechanism, but it seems unlikely that any do (65). Longer cells are better suited to utilize spatial sensing in a gradient, because the concentration differences between cell poles are greater. A description of a $6 \mu \mathrm{m}$ long marine Vibrio that likely utilizes this mechanism was recently published (269). Here it is hypothesized that two polar sensor patches located adjacent to flagellar motors control the rotational speed of the adjacent polar flagellum. By slowing one pole, the other pole travels farther per unit time resulting in reorientation of the cell in space. This mode of taxis is known as tropotaxis and leads to arching translational tracks (269). A similar mode of taxis in theory is possible in $B$. 
burgdorferi. There are polar flagellar motors (47), the cells swim in curves as well as straight lines (Figure 1.5), and there are likely receptor patches at each pole (87). While a molecular mechanism for such a system is not described, analysis of bacterial swimming behavior suggests it is possible

\section{Clinical manifestations of Lyme Borreliosis}

Lyme borreliosis is the most common vector borne disease in North America and Europe. The proven causative agents are 3 of the 11 species in the Borrelia burgdorferi sensu lato complex: Borrelia burgdorferi sensu stricto (s.s.), B. garinii, and $B$. afzelii. All are responsible for symptomatology in Europe and Asia while in North America Borrelia burgdorferi s.s., is the species responsible for Lyme disease (249). While human infections with $B$. lusitaniae $(55,138)$ and $B$. bissetti $(185,202,208)$ are documented they are rare and cause symptoms similar to those of $B$. burgdorferi s.I. All species are transmitted by the bite of haematophagous arthropods of the Ixodes ricinus complex. Clinical manifestations are varied and perhaps dependent on the infecting species (249).

\section{Disease Staging}

B. burgdorferi infection leads to a three-stage constellation of symptomatology. The first stage is demonstrated by 60 to $80 \%$ of patients and is characterized by erythema migrans (EM) lesions at the site of the tick bite. These are flat red lesions that begin as small macula or papule. These lesions, which often show central clearing, tend to migrate, thus the name erythema migrans. B. burgdorferi spreads laterally in the skin from the site of the tick bite, as evidenced by lesion spread and culture (140). Spirochete culture from these lesions is the gold standard for diagnosis and is approximately $90 \%$ sensitive $(249,251)$. Inflammatory mediators of the innate immune response commonly cause fever, chills, and malaise. Without treatment, lesions often regress but can return. If the infection goes untreated, manifestations of the second and third stages are seen $(249,251,252)$. 
The second stage is characterized by dissemination of the organisms. $B$. burgdorferi have been cultured from blood, myocardium, retina, muscle, bone, synovium, spleen, liver, meninges and brain. Although these sites are documented, skin is the predominate site of sequestration. Symptoms include migratory musculoskeletal pain, stiff neck, headaches, debilitating malaise, fatigue, and some degree of vascular damage are common. The most serious complication at this stage is hypercellular occlusion of vasculature (251 and references within). The knee is the most commonly affected musculoskeletal component followed by the elbow, ankle, shoulder, hip and temporomandibular joint. Up to $50 \%$ of untreated patients develop arthritis within two years of the EM lesion. Synovial fluid can show B. burgdorferi, B. burgdorferi DNA by polymerase chain reaction (PCR), elevated protein, and/or the presence of lymphocytes $(179,209,249,251)$. Of untreated patients, 15 to $20 \%$ develop neurologic symptoms and 4 to $8 \%$ cardiac symptoms. Early neurologic symptoms include aseptic meningitis, and palsies of the cranial and peripheral nerves. Cranial nerve VII is the most commonly effected, leading to a Bell's palsy. Any peripheral neuritis is asymmetrical and can be motor and/or sensory related. Electrophysiologic studies suggest a demyelinating process. Histologically, axonal injury is due to vascular injury of the nerve blood supply. Encephalitic symptoms, usually occurring later in infection, are often somnolence, poor memory, mood change and/or hallucinations. Musical hallucinations with patriotic or operatic overtones are reported (262). Neurologic symptoms can present at any time during the infection and will tend to have a relapsing and remitting course. Cardiac involvement in the form of atrioventricular heart block occurs in 1 to $4 \%$ of patients in North America, suggesting it is a $B$. burgdorferi s.s. disease manifestation. Acute myopericarditis, left ventricular dysfunction, or cardiomegaly can also result. Cardiac symptoms are usually transient and have a 3:1 female predominance $(179,251)$. 


\section{The third stage of the disease is likely due to an autoimmune response} triggered by $\boldsymbol{B}$. burgdorferi (173). This phase is characterized by chronic joint inflammation, often the knee, which does not respond to antibiotic treatment. Synovial exploration reveals villous hypertrophy with fibrin deposition and PMN infiltration. In severe cases, the inflammatory response will lead to bone and joint erosion. Several inflammatory mediators are released, including Ifn- $\gamma$, IL-13, IL-1 and $\mathrm{PGE}_{2}$. These inflammatory mediators activate collagenase and lead to proliferation of polymorphic neutrophils (PMNs), which can react to host antigens. Neurologic Lyme disease in the third stage is difficult to diagnose, as symptoms are subtle, appear after the more classic symptoms have disappeared, and antibody production in the CSF has ceased. Late neurologic symptoms are often paresthesias, spastic parapareses, bladder dysfunction, ataxia, and less commonly, radicular pain and/ or cognitive impairment $(173,179,191,210,239$, 251). The best described mechanism for autoreactivity comes from $T$ cell hybrids derived from individuals homozygous at HLA DRB1*0401. Using MHC class II tetramers, $10 \%$ of the population secreted inflammatory cytokines INF- $\gamma$ and IL-13 in response not only to $B$. burgdorferi outer surface protein A (OspA) but to leukocyte focal adhesion molecule $1 \alpha(\operatorname{LFA}-1 \alpha)(173,254,276)$. If the in vivo situation mirrors in vitro T-cell hybrid experiments, it would provide a mechanism for immune mediated Lyme arthritis. The current working hypothesis suggests that this is likely one of a number of autoreactive loci that plays a role. Knowledge of these loci are very important in vaccine development, as vaccination with an antigen that facilitates auto-reactivity is problematic.

\section{Immune Response}

The immune response to $B$. burgdorferi is humoral and cellular in nature. Early in the description of Lyme disease, a humoral response was noted and an "anti Ixodes spirochete" sera used as an investigational tool. It was noted that in the first 2 to 3 weeks post infection the humoral response produced IgM. After this point, an IgG response began to develop $(253,256)$. This early humoral response was to outer surface protein (Osp) C. OspC is one of a number of 
surface exposed proteins and is discussed in the section on gene expression. During Stage I, 20 to $50 \%$ of patients will mount a humoral response to $B$. burgdorferi antigens. During Stages II and III, this number is 70 to $90 \%$ or 90 to $100 \%$ depending on the patient population, respectively. As expected, OspC elicited not only a humoral response, but a cell mediated response (175 and references within, 205). Further investigation found the cell-mediated response to be $C D 4+T$ helper 1 , with little response from $C D 8+T$ cells, the $C D 4+T$ helper 2 response, or macrophages (63).

In addition to the adaptive immune response, the innate immune response is now recognized as important to host control of the infection as well as initial symptomatology. Toll-Like Receptor (TLR) 2 appears to be instrumental in the initial innate response to bacterial lipoproteins. TLR2 $2^{-1-}$ mice have normal humoral and cellular immune responses, yet have spirochete burdens 100 times that of $\mathrm{TLR}^{+/+}$littermates. Lipoprotein free fractions of sonicated spirochetes fail to cause signaling through TLR2, suggesting that a different surface associated molecule has some interaction with the receptor (174). Why TLR2 $2^{--}$mice have a deficient innate immune response is not well described, but failure to secrete matrix metalloproteinases in the absence of TLR2, or the general failure of polymorphonuclear leukocytes to recognize unopsoniated spirochetes has been suggested $(84,291)$. The abilities of the immune response are blunted by $B$. burgdorferi immune evasion mechanisms. Immune evasion ranges from antigenic variation to inducing host antigen presenting cells to stop expressing major histocompatabilty proteins $(15,149,150,241)$. Antigenic variation is discussed in the section on gene expression. The ability of $B$. burgdorferi to persist in tissues for long periods suggests that while the immune response keeps the infection controlled, it is not cleared (12).

\section{Serologic Diagnosis}

Reliable serologic diagnosis of Lyme disease was sought for more than a decade. As the body of data increased, the serologic response to B. burgdorferi 
became well characterized. Retrospectively, it was found that requiring 2 of the 8 most frequent IgM bands visualized on Western blot in early disease $(18,21,28$, $37,41,45,58$, and $93 \mathrm{kDa})$ and 5 of the 10 most frequent IgG bands $(18,21,28$, $30,39,41,45,58,66$, and $93 \mathrm{kDa}$ ) later in infection, enabled reliable serologic diagnosis of Lyme borreliosis. The IgM blot was $32 \%$ sensitive and $100 \%$ specific and the lgG blot was $83 \%$ sensitive and $95 \%$ specific (64). These results lead to a two-stage serologic diagnosis protocol. Any positive or equivocal primary ELISA (115) to a surface protein is sent for Western blotting where a positive reaction to 5 of 8 bands qualified as a diagnosis of Lyme borreliosis. Circulating immune complexes, rheumatoid factor, and anticardiolipin antibodies are also described $(179,249,251)$. Recently, recombinant variable major protein-like sequence expressed (VIsE) loci were described for diagnostic use. The VIsE proteins are usually described in relation to antigenic variation. However, a conserved portion of this molecule has proven as competent as the traditional two-step test for diagnostic purposes in an ELISA reaction. The epitope known as the $\mathrm{C} 6$ peptide is derived from the sequence of $B$. garinii. However this sequence is conserved across all borrelial species $(15,149)$. Conservation across species allows for diagnosis irrespective of where the infection was acquired, but does makes diagnosis less clear in patients with a history of infection with other Borrelia species.

\section{European and Asian Manifestations of Disease}

Borrelia garinii and B. afzelii are European and Asian isolates, the dermal manifestations of which are most commonly lymphocytoma cutis benigna or acrodermatitis chronica atrophicans (ACA), respectively. Interestingly, neither chronic articular manifestations nor the $\mathrm{AV}$ node cardiac conduction block are as common outside of the North America as within. This suggests differences in the disease caused by B. burgdorferi s.s. and those species found outside North America. The lymphocytoma is a soft nodule consisting of a dense polyclonal $B$ lymphocyte population of a few centimeters with diffuse borders generally in the pre-auricular, nipple or scrotum area. The lymphocytoma contrasts with ACA in 
that it spontaneously resolves. Acrodermatitis chronica atrophicans (ACA) is a bluish-red discoloration of the skin on the extensor surfaces of the hands and feet. Patient history is remarkable for the absence of prior history of symptoms. ACA has a characteristic slow progressive course with discoloration leading to atrophy of the skin. Organisms can be cultured from these lesions up to a decade after the onset of symptoms $(12,55)$. Radicular pain is more common in European and Asian patients, often in the abdomen or thoracic regions, as well as a disseminated encephalomyelitis that can be mistaken for multiple sclerosis (249 and references within). In sum, while the clinical pictures are similar in some ways between patients in North America and elsewhere, symptomology likely depends on infecting species. In the absence of an accessible animal model, it is difficult to show that different $B$. burgdorferi species cause unique symptoms unequivocally.

\section{Treatment and Vaccine}

Protective vaccines were developed that generated a humoral response to OspA, which is expressed by the majority of $B$. burgdorferi while in the tick gut. Blood containing an anti-OspA antibody can then opsonize $B$. burgdorferi in the tick gut preventing transmission. After three booster vaccinations of recombinant OspA, vaccinated adults were $92 \%$ less likely to become infected than the placebo group (240). The vaccine proved cost effective in populations where the incidence of Lyme disease was $1 \%$ a year or greater (206). However, when case reports of vaccinated individuals developing chronic arthritis began to appear, the vaccine was removed from the market for this and other reasons. A molecular mechanism to explain this vaccine side effect was described in the molecular mimicry of LFA-1 $\alpha$ and OspA $(173,254,276)$.

Standard treatment for Lyme borreliosis is doxycycline or amoxicillin for a period of 10 to 21 days. Lyme carditis and meningitis are often treated with ceftriaxone at $2 \mathrm{~g}$ per day for 10 to 21 days. Prophylaxis with $200 \mathrm{mg}$ of doxycycline within 72 hours of Ixodes tick removal is $87 \%$ effective at preventing Lyme disease 
(178). There has been a question about treating the chronic arthritis with continued antibiotic therapy. Randomized control trials have shown this not to be effective (133), further suggesting later stages of disease are immune mediated. It is important that treatment address not only $B$. burgdorferi, but also other organisms that are often tick-borne. Doxycycline is the preferred method of treatment for non-pregnant women because it also treats Ehrlichia chaffeensis or Anaplasma phagocytophila, the causative agents of ehrlichiosis, Coxiella burnetii the causative agent of $Q$ fever, and Rickettsia rickettsii the causative agent of Rocky Mountain Spotted Fever. These are important because they can have grave clinical consequences and have some symptoms in common with Lyme disease. In the case of Ehrlichia chaffeensis, these organisms are known to coinfect ticks with $B$. burgdorferi $(62,257)$. Babesia microti also co-infects Ixodes ticks, but usually this red blood cell parasite does not cause as severe a disease. If treatment is necessary, atovaquone-azithromycin combination therapy is effective (257). In sum, while early Lyme disease is easily treatable, diagnosis can be difficult and the sequelae of a missed diagnosis can be serious.

\section{Chemotaxis and Motility as Virulence Factors}

Chemotaxis and motility are hypothesized to be involved in the pathogenesis of a number of prokaryotes. Their exact roles in pathogenesis are becoming more rigorously described with new data. Flagellar structures, used for motility, often bind host tissues, and are major immunogens. Chemotaxis structures are largely intercellular or periplasmic, making their study less straightforward.

Pathogenicity studies utilize animal models, tissue explants, microscopy, recombination based in vivo expression technology (RIVET), in vivo-induced antigen technology (IVIAT), and DNA microarrary to profile protein expression during different stages of infection in a wide variety of prokaryotes $(100,118$, 141) 


\section{Chemotaxis and Motility as Virulence Factors in B. burgdorferi}

$B$. burgdorferi protein expression analysis and ecology suggest motility and chemotaxis are vital to the life cycle of $B$. burgdorferi. This pathogen must move from an array of tissues to the skin in order to infect a feeding tick and then back into a mammal upon the second feeding almost a year later $(19,38,116,143)$. It is doubtful these transitions that are required for pathogenesis could be made without a well functioning chemotaxis and motility system. B. burgdorferi motility and chemotaxis differ from the E. coli model genetically and structurally as described in a previous section. For an organism with a relatively low genetic capacity to maintain duplicates of chemotaxis and motility genes that are then constitutively expressed indicates the importance of motility and chemotaxis to the $B$. burgdorferi life cycle.

Motility almost certainly plays a role in the pathogenesis of this organism. While confirmatory experiments are not documented, non-motile mutants are likely nonpathogenic (219). Assays measuring the invasiveness of the organism demonstrate that $B$. burgdorferi binds to the extracellular matrix and can penetrate epithelial cell layers $(54,83,238,265)$. Similar assays using chemotaxis mutants await. The structure of a spirochete, as discussed in the structure and motility section, is well suited to swimming in viscous gel like media such as connective tissue. This observation correlates clinically to the ability of B. burgdorferi to spread through the dermis and to internal organs $(250,265)$. As discussed later in the introduction, genetic evidence, as well as data collected in vivo and in vitro, indicates that motility and chemotaxis are vital to the life cycle and pathogenesis of this organism.

\section{Chemotaxis and Motility as Virulence Factors in Other Species}

Vibrio cholerae pathogenesis has perhaps the best-described link to chemotaxis and motility. Because motility structures are antigenic and expressed in vivo, they are of interest as possible vaccine candidates. Additionally, chemotaxis 
and motility genes of Vibrio cholerae are linked with co-expression to toxin genes. Genetic studies reveal associations between the expression of toxin genes and chemotaxis associated genes. In motility mutants, toxin gene expression is aberrant (141). Genomic studies using DNA microarray show that chemotaxis genes are down regulated in stool derived cultures, suggesting that dissemination from the host requires or is promoted when chemotaxis is repressed (170). Studies looking at stool cultures are important because humans are the only natural hosts for $V$. cholerae. IVIAT data demonstrates that the hosts generate antibodies to pili proteins, Mcps, quorum sensing genes, and chemotaxis proteins, suggesting these proteins are expressed at some point during infection (100). RIVET in an animal model demonstrated chemotaxis mutants were better able to attach to small intestinal cells than the wild type (2.2 $-5.3 \mathrm{x}$ ), while flagellar motor mutants were less than $1 / 10$ as infective as the wild type (141). These results suggest that chemosensory structures are likely used outside the mammalian host while flagella are used in vivo for attachment. Data suggest chemotaxis and motility are necessary for various phases of the $V$. cholerae life cycle, but tight regulation of component expression is necessary for a fully pathogenic phenotype.

Helicobacter pylori is the causative agent of the majority of peptic and duodenal ulcers and infects more than $50 \%$ of the worlds population. Non-motile $H$. pylori cells are less likely to colonize host gastric tissues and are unable to establish long-term infections. Mcp mutants or other signal transduction cascade components are variably pathogenic in a mouse model. Co-infection studies reveal that wild type cells out-compete chemotaxis mutants in establishing infections, demonstrating the importance of chemotaxis in $\mathrm{H}$. pylori infection (9 and references within). Pharmacologic evidence suggests that motility plays a role in $H$. pylori infections in humans. Gastric ulcers are often treated with proton pump inhibitors like lansoprazole or rabeprazole to decrease acid production in the stomach aiding in the healing of the ulcer. Additionally, they inhibit the motility of $H$. pylori at lower concentrations than is required to inhibit growth 
(279). By inhibiting motility, these drugs likely lower microbial burdens by allowing for washout of $H$. pylori. While understanding of this model is still developing, data suggests that chemotaxis and motility are vital to this gastric pathogen.

\section{Chemotaxis and Motility as Virulence Factors in other Spirochete Species}

The invasive tissue penetrating nature of spirochetes has lead to conjecture that chemotaxis and motility are virulence factors. However, due to difficulty manipulating these organisms they are less well described in comparison to other bacteria. Nearly half a century ago Leptospira icterohemorrhagiae motility was implicated in the spread of organisms from guinea pig dermal sites to internal organs (250). Later work found pathogenic Leptospires chemotactic toward hemoglobin while an avirulent line of the same strain was nonchemotactic toward hemoglobin (295). The invasive oral spirochete Treponema denticola is implicated as the causative agent of gingivitis. Mcp and CheA mutants of $T$. denticola show markedly reduced tissue layer penetration in vitro. Aflagellate mutants demonstrate no tissue invasion (153) (156). While data from similar tissue penetration experiments with $B$. burgdorferi are not available, preliminary data suggested chemotaxis attenuated mutants would be less able to penetrate monolayers.

Brachyspira (Serpulina) hyodysenteriae is the spirochete species where the relationship between chemotaxis and pathogenesis is best described. It is of veterinary significance as the causative agent of swine dysentery. Predictably, it resides in the gastrointestinal tract and leads to bloody diarrhea. While it does colonize humans, infection is often sub-clinical and non-invasive $(114,127)$. Cells are specifically chemotactic toward hog gastric mucin, while gastric mucin of other species does not act as a chemoattractant. Single flagellar protein mutants, those in flaA and flaB1, while motile, only transiently colonize the mucosal surface. This transient colonization was likely due to flow through the porcine Gl tract and not actual binding. A double mutant in flaA and flaB1 
showed limited motility and very limited colonization. These data support the hypothesis that motility-attenuated strains are less virulent than non-chemotactic strains $(125,216)$. In sum, data generated with spirochete species support the notion that motility and chemotaxis are vital to completing a cycle of infection by allowing for transmission from vector to host, entry and establishment of a niche, and spread to new tissues and hosts.

\section{E. Borrelia burgdorferi Life Cycle and Gene Expression}

\section{Life Cycle and Vectors}

As already stated, the ticks that transmit the $B$. burgdorferi sensu lato family are all part of the same complex. The deer tick of the lxodes ricinus complex transmits $B$. burgdorferi s.I. In North America Ixodes scapularis (previously Ixodes dammini) is the predominate vector in the Midwest and the Northeast. Ixodes pacificus is the major vector in the West. In Europe, B. burgdorferi s.I. are usually transmitted by Ixodes ricinus, and in Asia Ixodes persulcatus is the major vector (249 and references within). These ticks provide the basis for transmission to humans.

The life cycle of $I$. scapularis is divided into the larvae, nymph, and adult stages. The small ground dwelling larvae rarely feed on larger mammals, restricting themselves to the accessible white-footed mouse (Peromyscus leucopus). The nymph stage is largely responsible for transmission to humans. The nymph's small size allows it often to go unnoticed, while feeding for the required 48 to 72 hours. The long feeding time is required for transmission from infected mouse to the tick to take place. Infected nymphs that fed on infected mice as larvae transmit the infection to larger mammals during feeding $(167,290)$. Adults feed on deer (Odocoileus virginianus) that are incompetent $B$. burgdorferi hosts. However, deer are the main food source for adult Ixodes ricinus ticks and necessary for the maintenance of the tick population (267 and sources within). 
In western North America, I. pacificus feeds on lizards that are incompetent hosts for $B$. burgdorferi. Infection is maintained by horizontal transmission of the infection through the dusky-footed woodrat (Neotoma fuscipess) and Ixodes neotomae, which does not feed on humans. It is when an errant I. pacificus feed on a woodrat infected by a I.neotomae bite does the possibility of human transmission occur. This horizontal route of infection keeps infection rates in western North America to $3 \%$ of what they are in the Northeast $(36,162,167$, 290). It is the best defined, but not unique, horizontal transmission route that maintains the infection in nature.

Maintenance of $B$. burgdorferi in nature requires a number of infection paths. Horizontal infection of small mammals by tick species is necessary to maintain $B$. burgdorferi in many environments. These horizontal transmission paths are important not only to the maintenance of $B$. burgdorferi in the environment but for the dissemination of $B$. burgdorferi sensu lato to new environments. $B$. burgdorferi s.l. have managed to spread over the Northern hemisphere and to select locations in the southern hemisphere, likely while residing in the digestive tracts of Ixodes uriae, an ectoparasite of seabirds. Once on board a seabird, $B$. burgdorferi can be disseminated transcontinentally. Very few Ixodes species engorge on mammalian hosts for the required 48 to 72 hours for human transmission (203). However, many are competent B. burgdorferi hosts. Therefore, it is the spread of tick species that are not competent for human transmission that often introduce $B$. burgdorferi into new areas. Once native ticks feed off small mammals infected by traveling tick species, transmission to humans is possible. This enzootic infection cycle of sea birds is recognized as an important route of $B$. burgdorferi spread. Enzootic cycles are now recognized as relevant to human infection in many environments $(86,166,185-188)$.

\section{Gene Expression}

B. burgdorferi demonstrates differential gene expression. Expression of surface exposed proteins are well-understood and intensely studied due to their potential 
as vaccine candidates. These proteins also play a role in transmission of the spirochetes. The outer surface lipoproteins (Osp) A through $E$ are exposed on the surface of $B$. burgdorferi and have a number of postulated uses. Osp $A$ and $\mathrm{D}$ are postulated to be used in host cell binding, OspB for phenotypic variation for host immune evasion, OspC for expression for host immune evasion, and OspE binds complement regulator factor $\mathrm{H}$ for prevention of killing $(66,101,102,192$, 238,288 ). Other common surface expressed proteins include VIsE (variable major protein like sequence expression site), decorin binding proteins (DbpA), and $p 83$. These latter proteins are antigenically variable for immune evasion, used to bind host tissues, or of unknown function but stably expressed so they are useful in diagnosis, respectively $(101,108,111,169,196,217,283,296$, 297). During tick feeding, a majority of $B$. burgdorferi in the tick gut stop expressing OspA and begin expressing OspC. Hypothetically, this transition in protein expression allows the $B$. burgdorferi to detach from the tick gut, move to the salivary glands, and into the site of the tick bite. Expression of DbpA then likely allows binding of the spirochetes to decorin proteins on host collagen. Antigenic variation of the $v s / E$ loci then aid in evading the host immune response as the spirochetes spread. Protein expression changes are hypothesized to be triggered by contact with host cell proteins, temperature, $\mathrm{pH}$, and the presence of fatty acids $(34,58,88,109,183,184,213,214,227)$. Several mechanisms of gene expression control are utilized by $B$. burgdorferi including LuxS-mediated quorum sensing, ggGpp / RpoS-RpoN stringent responses, and Rrp2 response regulator which governs gene expression. Sigma factor control is limited due to the presence of apparently only two major sigma factors, $\sigma^{54}$ and $\sigma^{70}(37,58,79$, $108,258,259,294)$. By controlling gene expression, B. burgdorferi is not only able to adapt to host environments, it is able to facilitate transmission. However, tight control over surface expressed proteins is unlikely to be sufficient for transmission. We hypothesize transmission requires competent chemotaxis and motility systems. 
To identify $B$. burgdorferi virulence factors, DNA microarrays were performed to broadly profile gene expression in the tick and host adapted states. The variables that change in this transition are temperature, $\mathrm{pH}$, and contact with host-adapted proteins. The host-adapted state is modeled by placing cells in a dialysis membrane chamber (DMC) implanted in abdominal cavity of a rat. Unfed and fed tick states are modeled by culturing cells at $23^{\circ} \mathrm{C}, \mathrm{pH} 7.5$, or $37^{\circ} \mathrm{C}$ $\mathrm{pH}$ 6.8, while groups looking at a temperature shift alone will change the culture temperature from $23^{\circ} \mathrm{C}$ to $35^{\circ} \mathrm{C}$. Data pertinent to chemotaxis and motility genes show that cells from DMCs or a fed tick state show increased expression of certain chemotaxis and flagellar genes. Temperature increases alone do not lead to a similar increase $(34,184,213)$. Genomic analysis reveals that many of these genes share homology in an up-stream promoter region, suggesting that there is a concerted host adaptation response. Induction for chemotaxis genes is a modest 1.5 to 3.5 fold depending on the gene and the study. This is expected, given that many of these genes are expressed under the control of a constitutively active $\sigma^{70}$-like promoter $(76,79,82)$. In sum, the environmental signals play a pivotal role in gene expression however, the constitutive expression of many chemotaxis and motility genes suggests that these functions are so vital to the $B$. burgdorferi life cycle that these structures are maintained irrespective of the environment.

\section{F. Genetic Manipulation of B. burgdorferi}

Knowledge of $B$. burgdorferi genetics is advancing rapidly. The development of genetic tools was hindered in large part to $B$. burgdorferi's slow growth in culture. Advances in this area have occurred on five principle fronts: understanding the sequence and chromosome make-up, defining the mechanism of chromosomal replication, manipulation using insertional mutagenesis, construction of shuttle vectors for complementation, and lateral gene transfer through transposon and phage activity. 


\section{Chromosome Structure and Replication}

The $B$. burgdorferi complete genomic sequence became partially available in 1997 and was completed in 2000. It is one of three known bacterial species that have a linear chromosome and/or linear plasmids. The chromosome itself is 910,725 bases in length with a significant plasmid content (12 linear and 9 circular) of 611,000 bases and is diagramed in Figure $1.6(45,76)$.

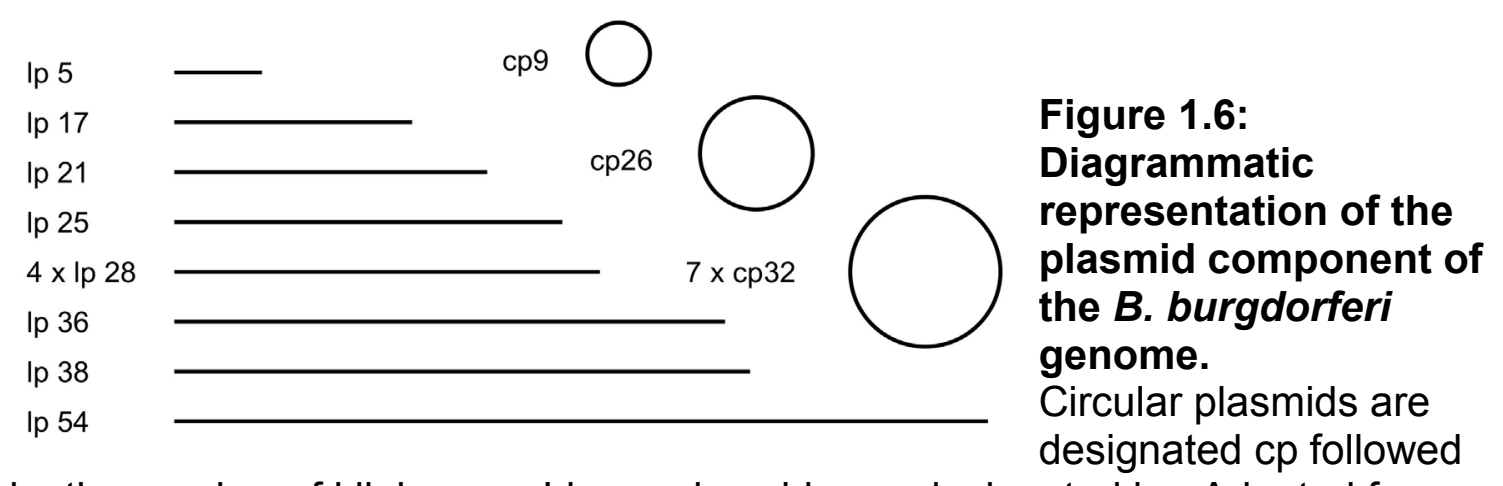

by the number of kilobases. Linear plasmids are designated Ip. Adapted from (45).

The $E$. coli genome is about 4 times larger than $B$. burgdorferi's suggesting greater significance of multiple copies of chemotaxis and motility genes ( $2 \mathrm{cheA}$, 3 cheW, 3 cheY, 2 cheB, and 2 cheR) (47). Replication of these linear structures was resolved when it was shown that a 140 bp region contained a site-specific DNA breakage locus that would convert a circular replicating form into a linear structure with closed hairpin ends (275).

\section{Genetic Manipulation}

Manipulating this genome proved difficult. The first selectable marker was gyrB ${ }^{r}$ that encodes a mutated form of the B subunit of the DNA gyrase gene that conferred resistance to coumermycin $A_{1}$. $(215,220)$. High frequency recombination with the endogenous gyrB gene limited the utility of this marker. More recently, kanamycin, erythromycin, streptomycin, and gentamycin resistance cassettes have become available, providing selectivity to high concentrations of antibiotics (27, 75 and references within, 223, 268). The 
development of resistance markers is limited to compounds that are not used clinically to treat Lyme borreliosis. With the recent development of these tools, our laboratory has inactivated most $B$. burgdorferi chemotaxis and motility genes (47).

\section{Shuttle Vectors and Complementation}

Recently, several B. burgdorferi shuttle vectors have been developed. Sartakova et. al. (2000) found that pGK12, derived from Staphylococcus aureus plasmids pE194 and pC194, containing erythromycin markers, with the replication functions from the Lactococcus lactis pWV01 plasmid, could propagate extrachromosomally in $B$. burgdorferi and support expression of green fluorescent protein (GFP) (221). Moreover, the pGK12 derivative successfully

complemented a B. burgdorferi motility mutant in cis (222). This $k a n^{R}$-containing shuttle vector is consistently propagated in $B$. burgdorferi. Because many mutants are constructed with the $\mathrm{kan}^{R}$ cassette, complementation requires a second reliable resistance marker. Samuels et. al. constructed the pFKSS1 shuttle vector that contains a hybrid spectinomycin / streptomycin resistance cassette in a pBSV2 backbone $(75,260)$. This hybrid resistance cassette allows for efficient selection in both $E$. coli and B. burgdorferi. Data in Chapter 3 demonstrate that pFKSS1 complements $B$. burgdorferi mutants constructed with the $\mathrm{kan}^{R}$ selection marker in trans.

\section{Lateral Gene Transfer: Transposons and Phages}

Lateral gene transfer is hypothesized to take place in $B$. burgdorferi. Hayes et. al. (1986) described particles found on electron microscopy consistent in size and shape to that of a phage (20). More recent work demonstrated a cell-free chloroform-resistant fraction was able to package and transduce DNA. Further study demonstrated that this was a temperate phage likely derived from one of the cp32s and was visualized microscopically (69). More controlled experiments demonstrated that $\phi \mathrm{BB}-1$, a bacteriophage of $B$. burgdorferi, was able to package cp32 and transmit kan ${ }^{R}$ to newly infected cells. This observation is likely of 
evolutionary importance because cp32 infects many different Borrelial species $(39,68)$. While there is little to no evidence of whole plasmid exchange, there is evidence of small fragment, less than $1 \mathrm{~kb}$, horizontal genetic exchange (66). These studies indicated that lateral gene transfer in $B$. burgdorferi is possible and likely a significant source of new genetic material in nature. Recently, Rosa et. al. generated a library of mutations through use of a transposon derived from a eukaryotic cell (unpublished data).

\section{G. The Structure and Function of Methyl Accepting Chemotaxis Proteins}

Methyl accepting chemotaxis proteins (Mcps) are the sensor molecules that control flagellar rotation and therefore chemotaxis (Figure 1.3). These proteins form membrane bound clusters, or are free in the cytoplasm, and are associated with the chemotaxis proteins $\mathrm{CheW}$ and CheA. Mcps function by sensing soluble molecules or light and facilitating taxis toward favorable environments (10). The binding of attractants to Mcps results in a conformational change of the molecule as well as leading to a subsequent decrease in autophosphorylation activity of the CheA.

\section{Mcp Structure and Function}

Mcps vary in number, cellular location, and ligand sensitivity depending on species. B. burgdorferi has five classical Mcps and one cytoplasmic Mcp (45, 76). E. coli has 4 classic membrane bound Mcps whereas Rhodobacter spheroides has $13(157,282)$. Cytoplasmic Mcps lack transmembane domains and are hypothesized to sense cytoplasmic chemistry or light, while membrane bound Mcps sample attractants in the external environment (10).

The ability of Mcps to sense chemoattractants astounded early observers. A 2 $\mu \mathrm{M}$ long $E$. coli would find a difference of 1 part in 10,000 between its nose and tail in an exponential gradient with a decay distance of $20 \mathrm{~mm}$. Furthermore, it can act on this difference in less than $2 / 10^{\text {ths }}$ of a second. This calculation was 
the basis for experiments that showed E. coli chemotaxis is dependant upon temporal and not spatial gradient sensing (159). The previously mentioned exception is a $6 \mu \mathrm{m}$ long marine Vibrio with bipolar flagella that appears to sense oxygen gradients spatially (269).

The structure of the Mcps directly relate to their function. The function of the Mcps is discussed in section B of this Introduction. Mcps are stable homodimeric helical structures that are inserted perpendicular to the membrane. Two sets of symmetric four helix domains sit outside the membrane, while two of the four domains span the membrane. On the cytoplasmic side, one helix from each member of the homodimer forms a hairpin. Ligands bind on the periplasmic side of the membrane in one of two sites formed by the interface of the homodimers. Currently, it is theorized that upon ligand binding there is a $1.6 \AA 5^{\circ}$ piston-like shift of the Mcp in the membrane that then interacts with the bound histidine kinase, CheA, to inhibit autophosphorylation activity $(7,72)$. This interaction stabilizes CCW flagellar rotation as outlined in the chemotaxis and motility section.

\section{Mcp Clustering and Amplification}

The location and spatial distribution of Mcps play a key role in their function. Membrane bound Mcps are located at the poles in most studied species of bacteria including Spirochaeta aurantia (87). Stoichiometrically abundant receptors (major Mcps) cluster with less abundant (minor Mcps) receptors at the poles. While less abundant receptors are polarly located they do not cluster in the absence of the abundant receptor species. These receptor clusters form patches in the range of 0.2 to $0.6 \mu \mathrm{M}$ in diameter; quite significant in a $2 \mu \mathrm{M}$ long E. coli $(72,142,157,158,199,237)$. This clustering occurs independently of CheW or CheA. In the absence of the Mcps, CheA and CheW proteins are found free in the cytoplasm $(157,164)$. The stoichiometry of Mcps, CheWs, and CheAs in these clusters is not well established. Workers have arrived at ratios from 1:1:1 to $7: 2: 1$. A widely accepted model uses the ratio of 2:1:1. A 2:1:1 ratio 
mathematically allows for tight packing of receptor clusters (28 and references within, 237). In mutants of minor Mcps, the cell is still chemotactic toward compounds detected by the major Mcps. If a gene encoding a major Mcp is mutated, then no signaling occurs from the minor Mcps or the major mutated Mcp (8). This suggests a model where an attractant binds to a few Mcps that leads to signaling through many Mcps. This amplification of attractant signals explains the high gain seen in chemotactic responses (245).

A second line of evidence for Mcp clustering and amplification comes from enzyme kinetics. The speed in which bacteria are able to sense and adapt to their environment is difficult to explain if only enzyme kinetics are examined. As polar clustering and the molecular structures of the proteins become better understood, a model is emerging to unify the empiric results. As receptors cluster in the membrane, their cytoplasmic tails act as inverted molecular tree trunks while CheW's and the bound CheA's form the canopy. In the space between the membrane and the canopy, the "adaptation compartment", CheR and $\mathrm{CheB}$ can function in close proximity to their substrates making them more effective than they would be free in the cytoplasm (Figure 1.7) (237).

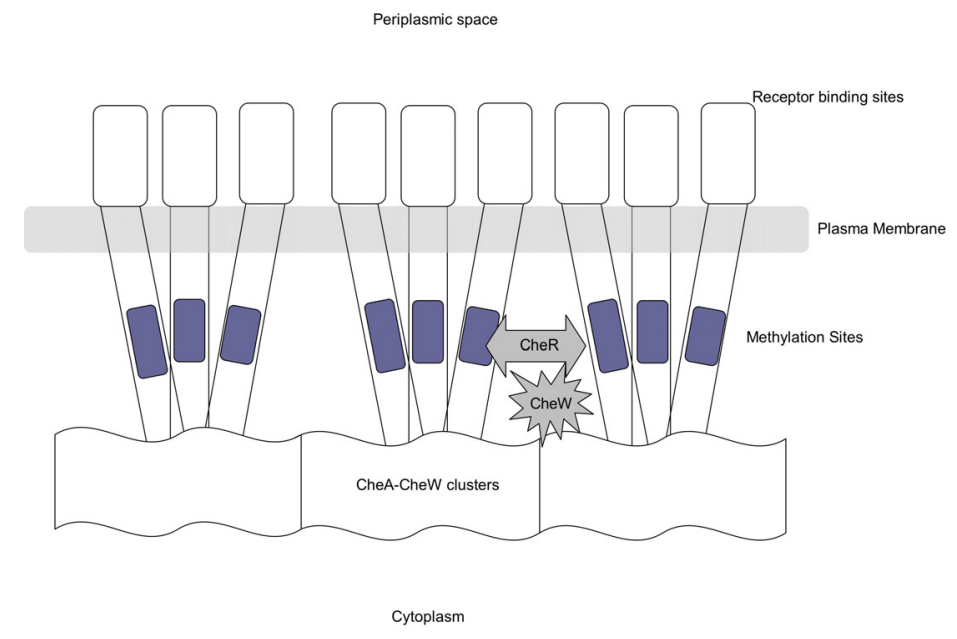

Figure 1.7: The Adaptation Compartment. CheR and CheW are hypotheized to exist in the space created by the plasma membrane and the bound CheA-CheW clusters. The close proximity of CheR and CheW to the Mcp methylation sites would speed methylation and demethylation reactions.

CheR is likely a brachiating molecule (142), traveling from one Mcp to another, amplifying the chemotaxis signal. Adapted from (237). 
Supporting evidence for this model was initially obtained from mutation studies. Mutants in CheR or CheB are not able undergo signal amplification. Furthermore, the approximately 850 copies of CheR per cell would have to methylate several clusters given there are approximately 4000 CheA and CheW molecules in the same cell (72 and references within). Structural analysis of CheR lead to the hypothesis that molecular brachiation allows CheR to move rapidly through a cluster, methylating the tails of the Mcps irrespective of their ligand specificity. The model incorporates signal amplification, the paradoxical data seen with mutants of major and minor Mcps, and the stoichiometry of signaling components. In silico testing of this model generated activation and adaptation data very similar to that published using cells $(72,130,142,237)$. Data generated using fluorescence resonance energy transfer (FRET) demonstrates the change in kinase activity (CheA) is approximately 35 times larger than the change in the number of attractant bound Mcps. Because FRET is able to measure protein-protein interaction in real time in vivo, it provides the most conclusive evidence to date that signal amplification at the level of the Mcps provides the gain observed in the chemotactic response (245).

\section{Mcp Response to Repellants}

Mcp activity toward repellants is not well understood but long observed. Pfeffer's original chemotaxis work in the 1860's included a description of positive and negative taxis (277). Negative chemotaxis, which occurs with sensitivities of 100 to 10,000 fold less than positive taxis, appears mediated by Mcps (277). Mcp null mutants are non-chemotactic both positively and negatively to specific stimuli. Mutants in the Mcp tsr (taxis toward serine) gene show impaired positive taxis to serine, yet also show impaired negative taxis to acetate, indole and Lleucine. Salmonella typhi and E. coli are very similar organisms, however, phenol is an attractant to the former and a repellant to the latter, yet toxic to both. This observation suggests repellant activity is not necessarily related to cell damage. Chemically, repellants cause the inverse of the attractant response. Repellants lead to a demethylation of the Mcps and possible increased 
autophosphorylation activity of $\mathrm{CheA}(94,242)$. Because $\mathrm{Ca}^{++}$concentrations play a key role in many prokaryotic and eukaryotic signaling systems, free- $\mathrm{Ca}^{++}$ concentrations were studied in relation to chemotaxis. The free $\mathrm{Ca}^{++}$ concentration was found to be inversely proportional to Mcp methylation. Attractants cause a drop in free- $\mathrm{Ca}^{++}$concentrations, while repellants cause an increase (274). How or if cytoplasmic free- $\mathrm{Ca}^{++}$concentrations effects rotational states the flagellar motors is unknown. Additionally, the change in configuration of the Mcps while a repellant is bound is undescribed. Receptors that sense solely repellants are not genome encoded. A chimera of a nitrate/nitrite receptor attached to the signaling domains of Tar (the aspartate sensing Mcp), generated a repellant signal demonstrating that a membrane bound receptor is able to transmit such a signal (286). While negative taxis occurs, likely through mechanisms shared or similar to that of positive taxis, the response is not well described.

\section{H. The Structure and Function of Chemotaxis Protein A (CheA)}

CheA is a homodimeric autophosphorylating histidine kinase. CheA is responsible for phosphorylating CheY leading to its interaction with flagellar motors to determine rotational direction (Figure 1.3 - starbursts). CheA exists in a long $\left(C_{h e} A_{L}\right)$ and short $\left(C_{h e} A_{S}\right)$ form as a consequence of an alternate internal initiation sequence. Usually these forms are found in a 1:1 ratio in the cell and null mutants in $\mathrm{CheA}_{\mathrm{s}}$ are phenotypically wild type. However, Che $\mathrm{A}_{\mathrm{s}}$ is required for the normal subpolar localization of CheZ and therefore possibly other proteins as well. $C h e A_{L}$ is not able to substitute for $C h e A_{S}$ in respect to this function (32, 43 and references within). CheA is well described, because genetic and functional evidence indicated it is a regulator of chemotaxis. Further description found its role fairly circumspect with phosphorylation of $\mathrm{CheY}$ and $\mathrm{CheB}$ as the major functions. 


\section{CheA Activation}

While activation of CheA awaits a detailed biochemical mechanism, much is known about its function. Activation occurs through a slight conformational change in the ternary complex, not association as the homodimeric structure might suggest. Binding of an attractant to a Mcp leads to suppression of this conformational change and therefore a decrease in activation $(85,104)$. The signal that leads to suppressed CheA autophosphorylation is integrated from several Mcps and amplified before it reaches the CheA. This integration includes an averaging of positive and negative stimuli from several Mcps and amplification at the level of CheR/B methylation/demethylation of the Mcp cytoplasmic tails. (8, 123, 130 and references within, 142, 245).

CheA structure is well described and is separated into 5 functional groups P1 through P5 (Figure 1.8). These groups are responsible for phosphorylation, CheY / CheB binding, dimer formation, ATP binding / catalysis, and CheW binding, respectively. These divisions have been further subdivided to study effects of specific residues on function.

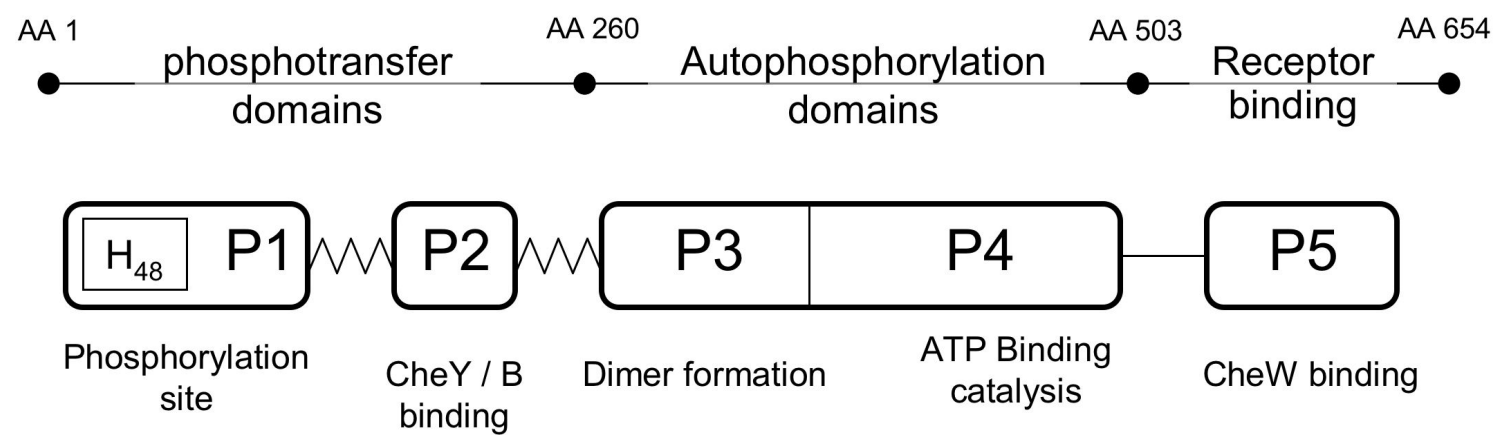

Figure 1.8: Diagrammatic representation of the functional sites in the CheA. adapted from Hirschman (104).

The $\mathrm{P} 1$ domain contains a histidine residue at position 48 that accepts the $\gamma$ phosphate from an ATP molecule held by the P4 subunit to become phosphorylated. This action is inhibited by the conformational change induced by attractant binding. P2 mediates the binding of $\mathrm{CheY}$ and CheB that are 
responsible for interacting with the flagellar motor to destabilize CCW default rotation, or returning CheA to the ground state respectively. P3 is responsible for holding the dimer of CheA together. The dimers likely cross phosphorylate making dimerization central to function. P5 binds CheW thus holding the CheA dimer in place. Without binding to $\mathrm{CheW}$, CheA floats free in the cytoplasm, binding CheY at will and thus competing with receptor bound CheAs. This competition is so successful that $\mathrm{CheW}$ mutants and strains that over-express CheA are non-chemotactic (85 and references within, 104, 181, 264).

\section{CheA in B. burgdorferi}

CheA species in $B$. burgdorferi are unique in that, there are two copies of the che $A$ gene widely spaced on the chromosome. These cheA genes likely evolved from different phylogenetic derivations. CheA1 and CheA2 show extensive homology to other CheA proteins with the $\mathrm{P} 2$ region (CheY / CheB binding) being less similar. Teleologically, this is expected because $B$. burgdorferi does not have a CheZ homolog but it has three CheY species. CheA2 shares $35 \%$ identity to CheA of Rhodobacter sphaeroides and is quite conserved among spirochetes. CheA1 shares $35 \%$ identity with the CheA of Vibrio cholerae suggesting it is a more recent acquisition. CheA1 mutants are able to reverse, in contrast to CheA2 mutants that constantly run and are non-chemotactic (144).

The presence of two cheA genes in $B$. burgdorferi is significant because of cell structure. As discussed in Introduction section B the sub-terminal polar motors of $B$. burgdorferi must rotate in opposite directions for translation to take place $(47,89,90)$. Therefore, it was possible that CheA1 and CheA2 localized to opposite poles playing a role in generating rotational asymmetry. However, $B$. burgdorferi cheA2::ermc and cheA2::ermc-cheA1::kan mutants constantly run, while cheA1::kan appears very similar to wild type (144). These results suggest an intrinsic polarity to the motors leads to different default rotational states of the bacterial motors and not the action of localized CheA species. 


\section{Capillary Tube Assay and Flow Cytometric Enumeration of B. burgdorferi}

The capillary tube chemotaxis assay was developed over a century ago $(2,4$, 172). It has been adapted to a wide range of prokaryotic species, and modified by different workers $(124,126,177,216,234,236)$. Our assay is based upon this prior work. This section will review the mechanics of the assay, basic chemotaxis principles, data reporting, and modifications made to measure the chemotaxis of $B$. burgdorferi.

\section{The Capillary Tube Assay}

The capillary tube assay measures how many bacteria swim into a capillary tube filled with a solution. This solution can be buffer alone or buffer plus a putative attractant or repellant. The number of cells that swim into attractant filled tubes is compared to the number of cells that swim into buffer filled control tubes at the termination of the assay. Data are reported as raw colony forming units (CFUs) per $\mathrm{ml}$ or as a factor of the number of cells entering buffer filled tubes.

Traditionally, cell pools were made by placing $U$ tubes on glass plates $(22 \times 28$ $\mathrm{cm}$ ) and the bottom of the U's covered by $22 \times 22 \mathrm{~mm}$ coverslips. U tubes were formed from capillary tubes $(1.10 \times 100 \mathrm{~mm})$ held with a hemostat over a Bunsen burner. Molten glass on either side of the hemostat fell, forming a $U .1 \mu \mathrm{l}$ microcapillary tubes fire polished at one end contained the solutions. The open end of the microcapillary tube was inserted into the cell pool and incubated for 30 minutes. The contents of these tubes were then added to molten LB agar and viable counts on pour plates performed $(1,171)$. This basic experimental design is adapted widely in the literature as noted above.

The capillary tube assay gave early insight into the nature of chemotaxis. Primarily, it demonstrated that chemotaxis is gradient dependent. The 
mechanism and rationale for this are outlined in the chemotaxis and motility section. Additionally, it provided an early measure of bacterial sensory capabilities. By testing the chemotactic response to increasing concentrations of attractant, it was shown that bacterial chemotaxis follows Weber's law. That is, that it takes a two-fold increase in concentration to elicit a response (171). This early observation was the basis for an important chemotaxis assay control. Although the cell senses an attractant, if there is no gradient of this attractant no chemotaxis will take place. Therefore, if a compound is truly a chemoattractant, the attractant response will be dose dependent. Assays with the same concentration of attractant in the cell pools as well as the tubes (the no gradient control), control not only for chemotaxis but also for a possible increase in swimming speed. If cells simply increased swimming speed in the presence of an attractant, more cells would be found in no gradient controls than in the buffer filled tubes. This scenario would lead to the recording of a false chemotactic response if data are reported as a factor over the buffer control (124).

\section{Modifications of the Capillary Tube Assay}

The capillary tube assay is widely adaptable. A number of modifications were made to the capillary tube assay for optimization. These included placing attractants in a viscous to semi-gel-like medium allows for attractants to diffuse out, and speeds or slows bacteria depending on the species. These assays can provide a qualitative measure of chemotaxis, or if the organisms are invasive, the organisms actually bore into a semi-solid substrate $(97,236)$. Other assay modifications have included chambers made of drilled Lucite blocks, $200 \mu \mathrm{l}$ pipette tips, 96 well plates, and in the case of $B$. burgdorferi, $2 \mathrm{ml}$ centrifuge tubes. Various sizes of capillary tubes and tuberculin needles have been used to contain buffer or attractant solutions $(59,95,124,168,177,281)$. Cells swimming into these tubes must be enumerated. For fast growing species, viable counts by plate count allow for quantification of live cells. A growth-based approach flushes cells into media filled 96 well plates and measures $\mathrm{OD}_{600}$ over 
a period of time. Comparison to known growth curves provides quantitative and easily automatable data collection (16).

Fastidious, slow growing organisms must be directly counted. The simplest method is Petroff-Hausser counting with a microscope counting chamber. However, this method is labor intensive, requires high cell densities for statistical reliability, does not easily differentiate live from dead cells, and suffers from inter observer bias. The approach used to enumerate B. burgdorferi in Chapter 3 is flow cytometry. Flow cytometry provides a high-throughput automated method for enumerating cells from capillary tube assays and provides data with a high degree of concordance to direct Petroff Hausser counts (42, 98, 139, 148, 243, 270).

\section{Enumeration of $\boldsymbol{B}$. burgdorferi using Flow cytometry.}

Fluorescence Activated Cell Sorting (FACS) and flow cytometry refer to similar technologies. An excellent explanation of the function and history of these technologies is given in Davey and Kell 1996 (61). FACS demonstrates the ability to physically separate cell populations, whereas flow cytometry demonstrates only the ability to count and visualize populations. Both technologies are able to resolve organisms based on light scattering and fluorescent characteristics. This technology was developed for clinical laboratories and proved quite capable of measuring cells in the 5 to $15 \mu \mathrm{m}$ diameter range. A few machines were developed for small particles, yet failed to find a market and are no longer produced (Bio-Rad Bryte HS, Hercules, CA; Skatron, Oslo, Norway). These machines were able to enumerate and even delineate different bacterial species based solely on light scattering characteristics (243). As sensitivities of detectors improved, measuring smaller particles, like bacteria, on clinically oriented commercially available flow cytometers became possible using selective dyes $(61,98,106,110,139,148$, $230,243,266,270$ ). Green fluorescent protein (GFP) expression in $B$. burgdorferi has been shown with flow cytometry but no direct enumeration or 
comparison to a known standard was performed (67). Chapter 3 remains the only report of flow cytometric enumeration of spirochetes.

To adapt ordinary flow cytometers to enumerate bacteria, procedural modifications are required. Scrupulous cleaning, calibration, and $0.2 \mu \mathrm{m}$ or smaller filtering of all solutions are necessary to minimize machine noise and background debris. For enumeration, most workers add nucleic acid dyes to delineate cells from debris. Some advocate the addition of polystyrene beads of a known size and concentration. This bead population will segregate distinctly from the cell population on flow cytometric analysis. This bead population can then be enumerated on a second gate acting as a control for flow rate (42). Our experience has suggested that while this might be a useful control, it is best not to gate absolute flow rate on bead parameters, due to bead clumping and other variables. Additionally, we found that $0.1 \mu \mathrm{m}$ filtering of all solutions and media was necessary to obtain the most reliable data.

\section{Flow Cytometric Enumeration of $B$. burgdorferi.}

Samples are prepared by diluting samples in $0.01 \mathrm{M} \mathrm{HEPES}, 0.15 \mathrm{M} \mathrm{NaCl}, \mathrm{pH}$ 7.4, containing $10 \eta \mathrm{M}$ fresh Syto-61 and $3-5 \times 10^{3} 6 \mu \mathrm{M}$-diameter quantitative polystyrene beads $/ \mathrm{ml}$. The addition of beads as an internal control was not always used. It gave very consistent results, but was plagued by quality control problems from suppliers. Because the bead control, when working, gave consistent results we believe the machine was consistently assaying the same volume of sample. Therefore, we chose to gate on flow rate. Syto61 is a membrane permeable nucleic acid dye giving a fluorescent signal readable on channel FL4 (139). Bead enumeration acted as an internal control to ensure that the volume enumerated was consistent for each sample. Samples were run at a rate of $12 \mu \mathrm{l} /$ minute for 60 seconds. A Becton-Dickinson FACScalibur with $15 \mathrm{~mW}$ air-cooled argon and red diode lasers operating at $488 \mathrm{\eta m}$ and $635 \mathrm{\eta m}$ respectively was used for all counting procedures. No compensation was necessary, as there was no spectral overlap between detectors (42). 
Representative machine settings can be found in Chapter 3. Using this technique, cell enumeration data statistically identical to that found with direct Petroff-Hausser counting were generated (Chapter 3 ).

Flow cytometry is rapid, allows for many parameters to be measured from a single sample, examines individual cells, and does not rely upon cells being culturable. Because of these capabilities, flow cytometry is widely applicable in microbiology. Species identification based on staining characteristics is the most published application. Specific stains can measure membrane integrity, membrane potential, enzymatic capabilities, or the presence of respiration. These parameters give information regarding cell viability, cell division state, effects of antibiotics, or the success of gene transfer if the protein of interest is fluorescently tagged (61). Specific species in mixed populations can be identified with a high degree of specificity with fluorescent in situ hybridization (FISH) probes $(61,148,230$ and references within, 243, 270). These capabilities and characteristics are leading flow cytometry to be applied more broadly in bacteriology.

Enumeration of bacteria using flow cytometry is a very recent development. Data showing concordance with traditional counting techniques like Petroff-Hausser counter or plating are beginning to be published. Whereas most studies rely upon plating for the comparison study, we utilized Petroff-Hausser counting due to the 20-day incubation time to get viable counts of $B$. burgdorferi. Viable counts with rapidly growing species show nearly identical results to flow cytometric counts demonstrating a high percent of chemotactic cells were alive $(61,98,106,139,148,230,270)$. Due to the high throughput of the flow cytometry protocol, and the agreement with direct Petroff-Hausser counting data, flow cytometry was used as our primary enumeration method. 


\section{J. Cell Motility Tracking Procedures}

The study of motility requires methods to track the movement of cells. Several tracking methods are documented, none ideal for all tracking of all microorganisms. Early methods used variations on multiple film exposures using stroboscopic lamps $(24,90,248)$. Once video equipment became available for microscope use, hand tracing of longer tracks became possible $(46,90)$. Most recently, the use of computerized tracking systems have allowed for higher throughput and decreased intraobserver bias $(119,144)$.

\section{Film Based Tracking}

The earliest systems were developed in the 1970's $(24,248)$. These systems measured speed, tumble length (twiddles) and direction. They were able to track E. coli for approximately 30 seconds. Based on photographic film, a long exposure time was used with a stroboscopic lamp illuminating the microscope field. As cells swam and were incrementally illuminated, they formed dashed lines on the film while swimming and spots during a tumble (248) (Figure 1.9). By using a stage micrometer, and knowing the flash frequency of the lamp, one was able to count the dashes and calculate the length of swims and tumbles on photographic prints.

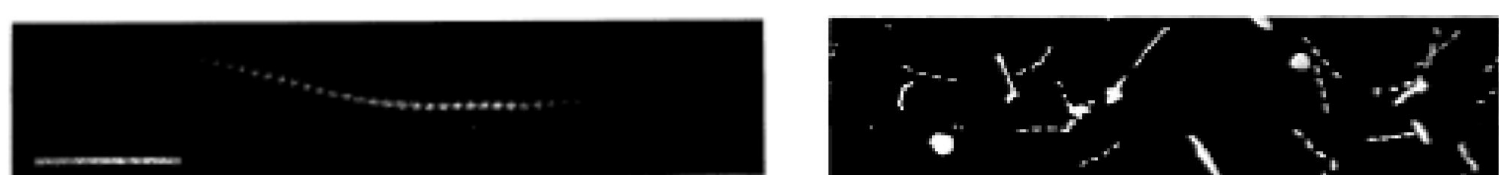

Figure 1.9: Examples of tracks of Leptospira illini (left) and E. coli (right). Both micrographs were taken using a long shutter time. L. Illini formed this pattern on film due to the screwing action of the spirochete swimming modality through methylcellulose under constant illumination. E. coli formed these tracks under stroboscopic illumination. Reproduced with permission from references. $(90,248)$.

The advantages to this system are that it requires little specialized equipment, allows tracking of many cells in a field, and is very sensitive for spotting changes in translational mode (i.e. runs to tumbles). It is problematic in that the length of tracks are limited by the number of exposures the photographic film will absorb, 
requires final measurements be done by hand, and works only for cells that move at least one cell length between exposures. The latter makes this approach difficult for use in spirochetes. Alternatively, using an automated camera to advance the film, a 4 second shutter time, and constant illumination this approach was used track Leptospira illini (Figure 1.9) (90). This micrograph shows that $L$. illini translated through a viscous gel-like media with little slippage, literally screwing through the methylcellulose. A similar method was adapted for use with video (235). These early methods allowed for the rigorous description of $E$. coli motility in the presence and absence of attractants.

Due to spirochetes elongated morphology photo based systems were less than ideal. The stroboscopic approach is not suited to a run-reverse-flex mode of translation. Because the cell moves over the same area many times, all tracks would form irregular spots. Additionally, data regarding reversal or stop frequency would be difficult or impossible to collect. These limitations lead to tracking spirochete motility by hand. With the marketing of video-microscopy equipment, cells could be taped. Video could then be analyzed frame-by-frame, tracing the cell path by marking on a piece of transparency film taped to the television monitor. While this approach was time consuming and labor intensive, it generated original data on the motility of spirochetes $(46,51,95,131,218)$.

\section{Computerized Tracking}

Due to the time consuming nature of hand tracking spirochetes, computerized methods were developed. These methods used commercial cell tracking systems in conjunction with software written especially to track video of swimming cells. This method allowed for greater throughput (100-200 tracks per experiment), was able to calculate reversal frequency, flexing frequency, and swimming velocity. Unfortunately, trackable film segments were limited to 15 seconds and were often as short 5 seconds, the software never became commercially available, and reversal frequency had to be verified by hand (74). This work demonstrated that automated tracking of spirochetes was possible and 
generated original data on the translational behavior of Spirochaeta aurantia (74).

Computerized tracking of cells underwent advances in the 1990's. Dr. Geoffery Hobson an engineering professor in Great Britain, developed a hardware/software package capable of tracking cars from video of busy intersections. This package quickly found an application with sperm motility researchers. From the sperm community, the prokaryotic motility community began to adopt this instrument. It is capable of tracking cells, stops, runs, stop time, stop frequency, curvilinear velocity, straight line velocity, track linearity, and curvature rate for hundreds of non-intersecting cells per field as running averages. More remarkable than the amount of data collected is its capability of making these measurements in real time. This instrument is utilized by a number of laboratories worldwide for prokaryotic cell tracking $(99,105,119,224,292$, 293). The Hobson system, with modification to data collection procedures, was able to track B. burgdorferi (Chapter 2) (144). The protocol is labor intensive, requires a spirochete to be alone in a field, translating quickly, and appear very bright on a very dark background. This system was only able to suggest where reversals took place, would only work with analogue images at 30 frames per second, and was less than user friendly. These limitations led to continued interest in new automated tracking instruments.

Recently, a software package made by Improvision Inc. has been able to track spirochetes with a much more user-friendly interface (Figure 1.5). Background movement is easily removed by cropping video images, and automated selection of moving objects. This makes almost any film suitable for tracking, and it decreases selection bias in the tracking of cells. Multiple spirochetes can be tracked in the same field, and data are reported on individual cells. The system is relatively inexpensive, and has an intuitive, easy to use, interface. However, this system is not able to track in real time, reversals must be verified by hand, the calculation period can last hours, requires a huge amount of RAM, and 
requires that files be in a proprietary .LIFF format. While no prokaryotic tracking is published using this software, our lab has found it easy to use, thus allowing several members of the lab to become proficient in tracking.

\section{Prokaryotic Translational Velocity}

A number of factors affect the speed of bacterial motility. Temperature, media viscosity, cell size, nutrient availability, and number of expressed flagella all correlate to changes in bacterial translational velocity. Table 1.1 gives an overview of the range of reported velocities of different bacterial species.

\begin{tabular}{|c|c|c|c|c|}
\hline Species & $\begin{array}{l}\text { Reported } \\
\text { low } \\
\text { velocity } \\
(\mu \mathrm{M} / \mathrm{sec})\end{array}$ & $\begin{array}{l}\text { Reported } \\
\text { high } \\
\text { velocity } \\
\text { ( } \mu \mathrm{M} / \mathrm{sec})\end{array}$ & $\begin{array}{l}\text { Reported } \\
\text { average } \\
\text { speed } \\
(\mu \mathrm{M} / \mathrm{sec})\end{array}$ & Reference(s) \\
\hline Azospirillum brasilense & 13 & 23 & & $(298)$ \\
\hline Bdellovibrio bacteriovorus & & & $\sim 200^{\nabla}$ & $\begin{array}{l}(212,261,271) \\
(91,131,144)\end{array}$ \\
\hline Borrelia burgdorferi * & 4.2 & 35 & & This work \\
\hline Campylobacter jejuni & 29.08 & 52.82 & 38.76 & $(119)$ \\
\hline Escherichia coli & 8.2 & 18.04 & 12.73 & $(24,119)$ \\
\hline Helicobacter pylori & 12.07 & 29.07 & 25.03 & $(119)$ \\
\hline Leptospira interrogans* & & & 30 & $(96)$ \\
\hline Proteus mirabilis & 10 & 45 & & $(226)$ \\
\hline Pseudomonas aeruginosa & & & $51.4+/-8.4$ & $(235)$ \\
\hline Pseudomonas fluorescens & 30 & 200 & & $(226)$ \\
\hline Rhizobium lupini & & & $37.9+/-5.4$ & $(224)$ \\
\hline Salmonella typhimurium & & & 55 & $(160)$ \\
\hline Spirillum serpens & 22 & 38.5 & & $(40)$ \\
\hline Spirochaeta aurantia * & 23 & 30 & 26 & (95) \\
\hline Treponema denticola * & 11.82 & 28.1 & 19.31 & $(218)$ \\
\hline Vibrio alginolyticus & 20 & 40 & & (13) \\
\hline Vibrio cholerae & & & $75.4+/-9.4$ & $(235)$ \\
\hline
\end{tabular}

Table 1.1: A summary of published translational velocities for selected prokaryotes.

* indicates spirochete species. ${ }^{\nabla}$ This velocity is based on a $2 \mu \mathrm{m}$ cell traveling at 100 cell lengths per second. 
While some spirochetes have slower translational velocities than many species, they are able to swim in environments that slow or stop most externally flagellated organisms. The spirochetes' helical or flat wave morphology generally increases translation speed in viscous media $(40,90,91,131,218)$. Therefore, the range of translational speeds is dependant upon media conditions. There are two types of viscosity, macro and microscopic. Gels are macroscopically viscous indicating that a molecular mesh forms allowing cells to push against this mesh as it moves. Methylcellulose or gelatins are the classic examples of media additives that increase the macroscopic viscosity. Increasing both the microscopic and macroscopic viscosity can be done with the addition of compounds like Ficoll. It is suggested that viscosity alone can lead to a positive chemotactic response, but this has not been definitively shown (201), and is required for the motility of some species (218). While bacterial translation speeds vary widely, spirochetes are especially suited for motility in environments that slow or stop most species. 
This work was published as:

Li, C., R. G. Bakker, M. A. Motaleb, M. L. Sartakova, F. C. Cabello, and N. W. Charon. 2002. Asymmetrical flagellar rotation in Borrelia burgdorferi nonchemotactic mutants. Proc Natl Acad Sci U S A 99:6169-74.

\section{Chapter 2: Asymmetrical flagellar rotation in Borrelia burgdorferi cheA} mutants

Chunhao Li, ${ }^{*}$, Richard G. Bakker*, Md. Abdul Motaleb*, Marina L. Sartakova , Felipe C. Cabello , and Nyles W. Charon* $\ddagger$

* Department of Microbiology, Immunology, and Cell Biology, Health Sciences Center, Box 9177,West Virginia University, Morgantown, WV 265069177

Department of Microbiology and Immunology, New York Medical College, Valhalla, New York 10595.

Running title: Spirochete Borrelia burgdorferi chemotaxis

${ }^{\ddagger}$ Corresponding author. Mailing address, Department of Microbiology, Immunology, and Cell Biology, Health Sciences Center, Box 9177, West Virginia University, Morgantown, West Virginia 26506-9177. Phone: (304) 293-4170. Fax (304) 293-7823. Electronic mail address: ncharon@wvu.edu

Key words (spirochete/periplasmic flagella/Borrelia, Lyme disease, chemotaxis, motility) 


\section{Abstract}

The histidine kinase CheA is an essential component in the signaling pathway for bacterial chemotaxis. Mutants of $c h e A$ in flagellated bacteria continually rotate their flagella in one direction. In the Lyme disease spirochete Borrelia burgdorferi, bundles of periplasmic flagella are subpolarly located at each cell end. In this paper, we present evidence that che $A$ mutants of $B$. burgdorferi asymmetrically rotate their bundles of periplasmic flagella. $B$. burgdorferi has two copies of cheA designated cheA1 and cheA2. We found that both genes were expressed, and part of operons initiated by $\sigma^{70}$ promoters. These results further support the conclusion that $B$. burgdorferi is unique as it lacks a specific sigma factor to control motility gene expression. To understand the function of the two cheA genes, each was targeted by mutagenesis. cheA1::kan had no obvious phenotypic differences compared to the wild type. However, cheA2::kan swam only in one direction and was deficient in chemotaxis. We completely blocked the signaling pathway by constructing a double mutant cheA1::kan cheA2::ermC. This double mutant had the identical phenotype as the cheA2::kan mutant, and video microscopy of tethered cells revealed that the periplasmic flagella rotated at each cell end. The results indicate that the double mutant resembles cheA mutants in other bacteria by its constantly running phenotype. However, it differs with respect to flagellar rotation, as its default mode is rotation of the periplasmic flagella bundles in opposite directions. The results indicate an asymmetry with respect to flagellar rotation in spirochetes. 


\section{Introduction}

Bacterial chemotaxis is a complex sensory transduction pathway that enables cells to respond to environmental stimuli. Cells swim toward a favorable medium, or away from one that is toxic. A two-component phosphorelay system plays an essential role for this response. The most well studied systems are Escherichia coli and Salmonella enterica serovar Typhimurium. A family of chemoreceptor proteins regulates the autophosphorylation of the sensor histidine kinase CheA. Activated CheA phosphorylates the response regulator $\mathrm{CheY}$, which then interacts with the switch complex at the flagellar motor to change direction of flagellar rotation from counterclockwise (CCW) to clockwise (CW). CCW rotation results in smooth swimming cells, and $\mathrm{CW}$ rotation results in tumbling. Null mutants in cheY or cheA continuously rotate their flagella CCW and consequently fail to tumble (194). Cells showing a positive chemotactic response have longer runs and suppress the time spent tumbling $(10,32,71,264)$.

Spirochetes are a structurally unique group of bacteria. The organelles for motility, the periplasmic flagella (PFs), reside between the outer membrane sheath and cell cylinder. These PFs are subterminally attached at each end of the cell cylinder and propel the spirochetes by rotation $(48,91,146)$. In the Lyme disease spirochete $B$. burgdorferi, 7-11 PFs are subterminally attached at the cell poles and form a bundle. These PFs overlap in the center of the cell and form a continuous ridge from one cell end to the other (89). During swimming, these organisms run, pause, and run again either in the same or opposite direction. They also reverse direction without any detectable pausing $(91,234)$. Pausing is accompanied with a major change in shape, often bending at the cell center, and is referred to as a flex (91). Recent models of $B$. burgdorferi motility indicate that 
in translating cells, the anterior PFs rotate $\mathrm{CCW}$, and the posterior PFs rotate CW as viewed from the end of the PF filaments along their shaft $(89,146)$ (Figure 1).

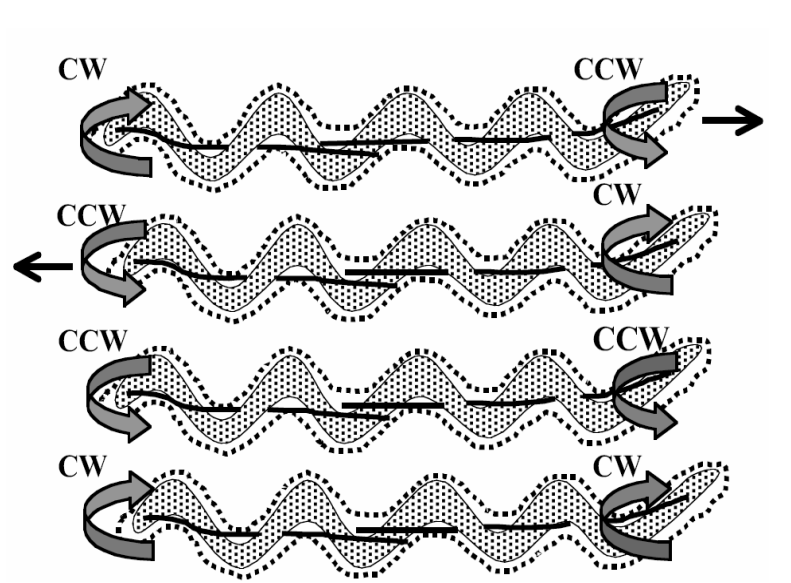

Figure 2.1: Swimming cells of $B$. burgdorferi as a function of direction of rotation of the periplasmic flagella. Black arrows indicate direction of swimming. Dotted lines represent the outer membrane sheath. Grey arrows indicate direction of rotation of the periplasmic flagella (thin lines). For simplification, only one periplasmic flagellum is shown attached at each end of the cell cylinder. In B. burgdorferi, there are between 7-11. The top two diagram translation forms, and the bottom two diagram non-translational forms leading to a flex.

Interaction of the rotating PFs with the cell cylinder cause backward propagating waves to be generated along the entire length of cell. Flexes result from the bundles of PFs rotating in the same direction $(89,91,146)$.

We know very little about the chemotaxis of spirochetes. In contrast to the chemotaxis system of other bacteria, a membrane potential mediates the chemotactic response in Spirochaeta aurantia (93). Several compounds and media components have been shown to serve as attractants and repellents for $S$. aurantia (95), and to a lesser degree for Treponema denticola $(121,147)$ and $B$. burgdorferi (234). Disruption of the cheA and chemoreceptor genes $d m c A$ and $d m c B$ in $T$. denticola results in cells that fail to penetrate monolayers of eukaryotic cells $(153,154)$. Genomic and transcriptional analysis of $B$. burgdorferi indicate that it has several clusters of motility and chemotaxis genes, 
some of which function as operons $(76,79,146)$. Multiple copies of chemotaxis genes are present including two copies of $c h e A, c h e B$, che $R$, and three copies of cheW and cheY. Most of the chemotaxis genes are located within two clusters that map far from one another. In addition, within these gene clusters there are several open reading frames that show no homology to motility and chemotaxis genes of other bacteria. One well-studied cluster is referred to as the fla $A$ operon, which consists of flaA, cheA2, cheW3, cheX, and cheY3 (Figure 2a) (78, $80,81)$. Primer extension and RT-PCR analysis indicates that this cluster comprises an operon and is initiated with a $\sigma^{70}$-like promoter sequence. These results are consistent with other transcriptional and genomic analyses that indicate that $B$. burgdorferi is unique in its control of motility and chemotaxis genes; the four motility and chemotaxis operons characterized to date are under $\sigma^{70}$ control $(79,80)$. The other less studied group of chemotaxis genes is the cheW2 cluster (Figure 2b). It consists of cheW2, orf566, cheA1, cheB2, orf569, and cheY2. In this communication, we carried out transcriptional start site and RT-PCR analyses of the cheW2 gene cluster. The results obtained indicate that this gene cluster also comprises a $\sigma^{70}$-like initiated operon.

Recent improvements in the genetic manipulation of spirochetes have allowed for targeted mutagenesis $(27,221,272)$. We have shown that targeting the major flagellar filament gene flaB results in cells that lack PFs, are non-motile, are no longer wave-like but rod-shaped (176). To further understand the function of individual chemotaxis and motility genes in this species, we isolated single deletion mutants in the cheA1 and cheA2 genes, and a double deletion cheA1cheA2 mutant. The results indicate the cheA2 mutant and the double mutant behave like cheA mutants of other bacteria in that they constantly run in one direction. For this to occur, we propose there is a departure from the 
established chemotaxis paradigm whereby the flagella rotate in the same CCW direction in the absence of CheA. In B. burgdorferi that lack CheA, the PF bundles at each end of the cell rotate in opposite directions relative to one another.

\section{Materials and Methods}

Bacterial strains, growth and chemotaxis assay conditions, and plasmids.

A single clone of avirulent B31, referred to as B31-A, was used for all gene transfer experiments and served as the reference wild-type strain (27). The nonmotile PF- mutant MC-1, liquid BSK-II medium, agarose plates, growth conditions, and swarm agar plates have been previously described (176). Capillary tube chemotaxis assays were carried out similar to the method described by Shi et al. for B. burgdorferi (234). Assays were done using $50 \mu \mathrm{l}$ capillary tubes incubated for $2 \mathrm{hr}$ at $34^{\circ} \mathrm{C}$ in $3 \% \mathrm{CO}_{2}$ atmosphere. E. coli strains were grown in Luria-Bertani broth. 


\section{Plasmids}

pGME-T: Ampr, PCR cloning vector

pGA1: pGME-T Easy with 1,690 bp cheAl gene amplified by PCR

pGA2: pGEM-T Easy with 2,521 bp cheA2 gene amplified by PCR

pGAl kan: pGAl with $1.3 \mathrm{~kb}$ flgB-kan replacing $393 \mathrm{bp} \mathrm{HindIII/HindIII} \mathrm{fragment}$

pGA2kan: pGA2 with $1.3 \mathrm{~kb}$ flgB-kan replacing $1,326 \mathrm{bp}$ HindIII/HindIII fragment

pGA2ermC: pGA2 with $1.1 \mathrm{~kb}$ ermC replacing 1,326 bp HindIII/HindIII fragment

pQE31:Expression vector

pQE-CheAl: cheAl cloned into pQE-31 BamHI/PstI sites

DNA Primers:

5'-ATATTTAATCTCAATAGC-3' (cheW2 reverse, primer extension)

5'-AAGTAATTTCTGAGATCG-3' (cheAl, 5 ' +, inactivation)

5'-AAAGTCTGATAACAGTTCGG -3' (cheAl, 5' +, inactivation )

5'-TAGTTATTGCATCTATGTC-3' (cheA 1, 3'-, inactivation)

5'-ATGGAAATATTAGATTTGG-3' (cheA2, 5' +, inactivation)

5'-ATTTGGAAAATGAAGAGC-3' (cheA2, 5' +, inactivation)

5'-TTACCATTGCCAAGCGTAG-3' (cheA2, 3' -,inactivation)

5'-AAGCTTTAAT ACCCGAGCTTCAAG-3' (flgB-kan, $5^{\prime}+$, kan cassette)

5'-AAGCTTTCAAGTCAGCGTAATGCT-3' (flgB-kan, 3' -, kan cassette)

5'-AAGCTTAACACACTAGACTTATTTAC-3' (ermC, 5' +, erm C cassette)

5'-A A GCTTAAAAAATAGGCACACGAAAA-3' ( $\mathrm{ermC}, 3$ '-, $\mathrm{ermC}$ cassette)

5'-ACGGATCCGGATAGTAGTGATGTTATTG-3' (cheAl, 5' +, expression)

5'-CTGCAGTATAAGTTTAGTTATTGCATC-3' (cheAl, 3' -, expression)

* Italic indicate engineered restriction cut sites.

Table 2.1 Plasmids and sequences of primers.

This table contains the sources of plasmids and the sequences of primers used in these studies. bold italics indicates sites of engineered restriction cut sites.

DNA manipulation, PCR conditions, and primers. Restriction mapping, enzyme modification, and transformation were carried by standard procedures (14). For amplification of target genes, primers sequences and plasmids are listed in Table 1. Amplified products were purified using Qiagen PCR purification kits or gel removing kits. The resulting products were cloned into the respective plasmids for further manipulation. Alignment and DNA analysis was done using Peptool, GCG, and DNAasis. 


\section{Construction of plasmids, pGA1kan, pGA2kan and pGA2ery, and targeted} mutagenesis. che $A 1$ and cheA2 were amplified by PCR from chromosomal DNA, and the resultant products were cloned into pGEM-T Easy vector (Promega). Hindlll was used to generate an internal deletion in each cheA gene. The 393 bp deletion in che $A 1$ and the 1,326 bp deletion in che $A 2$ were each replaced with a $1.3 \mathrm{~kb}$ flgB-kanamycin resistance cassette (176). The plasmids obtained, pGA1kan and pGA2kan, were used as the source of DNA for targeted mutagenesis. To construct the plasmid pGA2ery, the HindIII generated deletion in cheA2 was replaced by the amplified erythromycin resistance cassette ermC (221). Restriction digest mapping indicated that the deduced direction of transcription of kan or ermC was in the same as that of cheA1 or cheA2. Preparation of competent B. burgdorferi, electroporation, and plating of transformants were done as previously described $(27,176)$. Approximately $2 \mu \mathrm{g}$ of amplified input DNA were used for electroporation. Growth media were supplemented with $350 \mu \mathrm{g} / \mathrm{ml}$ kanamycin, $0.05 \mu \mathrm{g} / \mathrm{ml}$ erythromycin, or as needed.

\section{RNA preparation, RT-PCR, primer extension, and construction of} recombinant proteins. Total RNA was prepared for both RT-PCR and primer extension analysis as previously described (78). RT-PCR was carried out by using the One Step RT-PCR kit (Qiagen). Primer extension was done using the AMV reverse transcriptase Primer Extension System (Promega) (79). B. burgdorferi CheA1 was over-expressed as a His-tagged fusion protein. The complete gene was amplified and cloned into PQE31 vector (Qiagen) at BamHI/Pstl sites, resulting in $\mathrm{pQE}-\mathrm{CheA1}$. This construct was transformed into host cell M15 (pREP4) (Qiagen). Cells readily overproduced CheA1 after induction with IPTG. The overproduced protein was purified with ProBond 
Purification System (Invitrogen) according to the manufacturer's instructions. Approximately $350 \mu \mathrm{g}$ of recombinant CheA 1 protein were used to immunize rats to produce specific antiserum. Antiserum to recombinant CheY 3 was made in a similar manner and will be described elsewhere (M. Motaleb, R. Bakker, C. Li, N. Charon, unpublished).

Gel electrophoresis and western-blotting. Sodium dodecyl sulfatepolyacrylamide gel electrophoresis (SDS-PAGE) and Western blotting with the ECL detection method (Amersham Pharmacia) were carried out as previously reported $(78,145)$. Rabbit antiserum to $E$. coli CheA was generously provided by P. Matsumura. Monoclonal antibodies to FlaA, FlaB, and DnaK were kindly provided to B. Johnson, A. Barbour, and G. Benach, respectively.

\section{Electron and light microscopy, and computer assisted motion analysis.} Standard methods for electron microscopy and negative staining were used to view spirochetes with attached periplasmic flagella (33). Live cells were observed by dark-field or phase microscopy using Zeiss optics with cells held at $34^{\circ} \mathrm{C}$ using a Physitemp temperature controlled stage (176). Video-recording of images was carried out as previously described (91). The Hobson BacTracker was used to track the motion of individual swimming cells (119). Cells were suspended in BSK II media a final concentration of $1 \%$ methylcellulose (91). Because of the large size of $B$. burgdorferi relative to its slow velocity, modifications were made for data analysis. Cells were videotaped using dark-field illumination at 200X for at least one minute. We used the $X Y$ module of the tracking system whereby the position of the center of a cell was determined every $1 / 60$ th sec. In order to obtain specific data on velocity and reversing, we averaged every 12 data points $(0.2 \mathrm{sec})$. The distance the centroid traveled between these averages were 
graphed as a 2-dimensional track and a bar chart of distance vs. time. In addition, the video was digitized allowing for frame-by-frame verification of run and reversal intervals. At least three cells were tracked for a given strain, and the results are expressed as mean \pm S.D. To analyze tethered cells, cells in BSK-II (without methylcellulose) that adhered to the glass in the central part of the cell were video-recorded by phase microscopy at $2500 \times \mathrm{g}(91)$.

\section{Results}

Transcriptional analysis of cheW2 operon. In our initial sequence analysis of motility gene clusters of $B$. burgdorferi, we hypothesized that most of the motility and chemotaxis genes in $B$. burgdorferi were identified and mapped (Figure 2.2) (91). We also found that many of these genes resided in operons that were initiated by $\sigma^{70}$ promoter sequences (146). However, the recent determination of the entire genome revealed the presence of multiple copies of chemotaxis gene homologs such as cheA, cheW and cheY. Many of these newly identified chemotaxis genes mapped within the cheW2 gene cluster that consists of cheW2, orf566, cheA1, cheB2, orf569, and cheY2 (Figure 2.2b) (76).

(a)

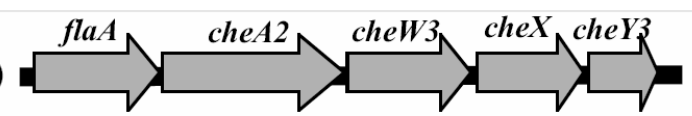

(b)

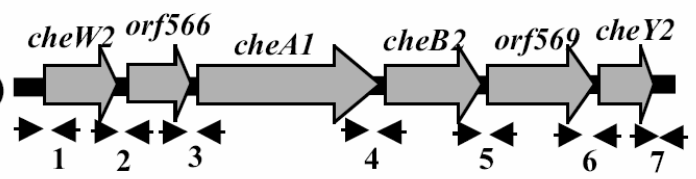

(c)

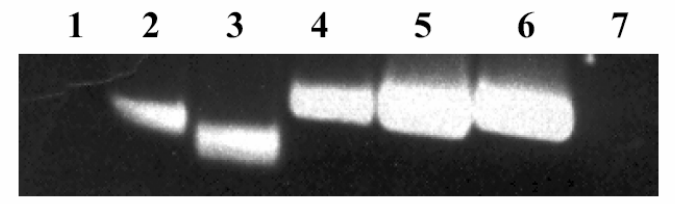

Figure 2.2: Genetic map of the flaA and (b) cheW2 clusters. Wide arrows indicate direction of transcription.

(c) RT-PCR results using primer pairs designated below map of cheW2 cluster.

orf =open reading frame che $=$ chemotaxis gene $\mathrm{fla}=$ flagellar gene

Analysis of this cluster revealed that there is little intergenic space between genes (range 5-68 bp) with no obvious promoters within and between the genes. 
RT-PCR analysis suggested that all six genes were co-transcribed as a polycistronic mRNA (Figure 2.2c). Primer extension analysis revealed a $\sigma^{70}$-like recognition sequence directly upstream of cheW2 (Figure 2.3a). This promoter was highly conserved at both the -10 and -35 regions with other motility and chemotaxis operons $(79,80)$ (Figure $2.3 b)$.

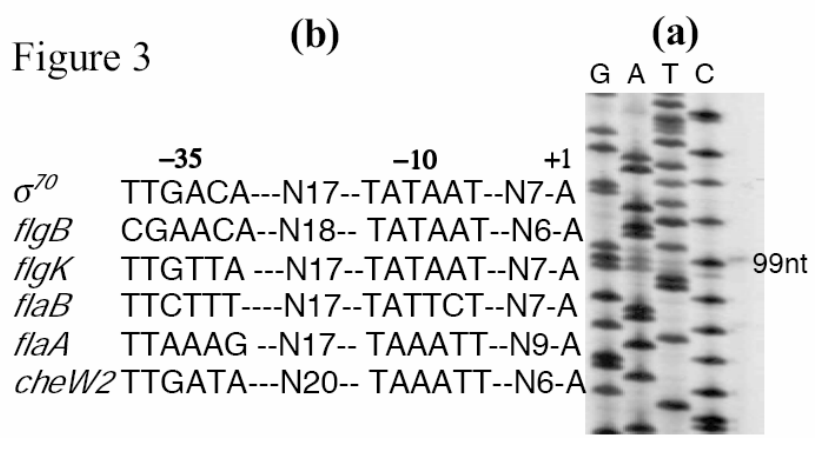

Figure 2.3 (a) Primer extension results of cheW2 cluster show a $\sigma^{70}$-like recognition sequence. (b) Similarity of $\sigma^{70}$-like promoter sequence of cheW2 to those of other motility and chemotaxis operons.

The -10 region (TAAATT) was

identical to the previously described flaA chemotaxis operon. These results indicate that the cheW2 cluster is initiated by a $\sigma^{70}$-like promoter similar to the other four motility operons.

Structure and alignment of CheA1 and CheA2. To gain insight into the function of CheA1 and CheA2, we compared their deduced amino acid sequences to each other and to their counterparts of other bacteria. The two che $A$ genes map far from each other on the linear $B$. burgdorferi chromosome: cheA1 at nucleotides 578277-580418, and cheA2 at nucleotides 706673-709264. The CheA amino acid sequences are 33 per cent identical. CheA1 and CheA2 have 33 and 34 per cent identity to $E$. coli CheA, respectively. Both $B$. burgdorferi CheA1 (79.4 kDa predicted size) and CheA2 (98.4 kDa predicted size) are larger than CheA of $E$. coli $(71 \mathrm{kDa})$. CheA from several bacterial are shown to consist of 5 functional domains $(\mathrm{P} 1 \rightarrow \mathrm{P} 5)$ joined by linker regions, as discussed in the Introduction $(26,71,264)$. We found that CheA1 and CheA2 
had extensive homology in each of these five domains, with less conservation in the region corresponding to $\mathrm{P} 2$. Alignment analysis indicated that $B$. burgdorferi CheA2 is most similar to CheA proteins of the spirochetes Treponema pallidum (40 per cent identity) and $T$. denticola (42 per cent identity) $(77,231)$. In addition, B. burgdorferi CheA2 and T. pallidum CheA share two large unique regions located at the putative P2 (323-339aa) and T domains (454-517aa). In contrast, B. burgdorferi CheA1 had its highest homology to that of Rhodobacter sphaeroides CheA2 (35\% identity) and Vibrio cholerae CheA1 (35\% identity) (45, 76). These results suggest that CheA1 is likely to be a recent acquisition from the Proteobacteria, whereas CheA2 is well conserved among the spirochete species. The Leptospira intrerrogans genome sequence recently became available, but has not been carefully analyzed from a genetic perspective (211).

Construction and analysis of LC-A1 and LC-A2 mutants. To examine the function of the $c h e A$ genes, we targeted each by allelic exchange mutagenesis with accompanying deletion formation. After electroporation and selection, PCR analysis indicated that the cheA1 (LC-A1) and the cheA2 (LC-A2) mutants each contained the kan insert. These inserts were transcribed in the same direction as cheA1 or cheA2. Western blot analysis was used to test for synthesis of both CheA1 and CheA2. Two different sources of antibodies were used in the analysis. First, an antiserum directed to $E$. coli CheA was found to react with a band corresponding to CheA2 in wild-type cells (Figure 4a). 


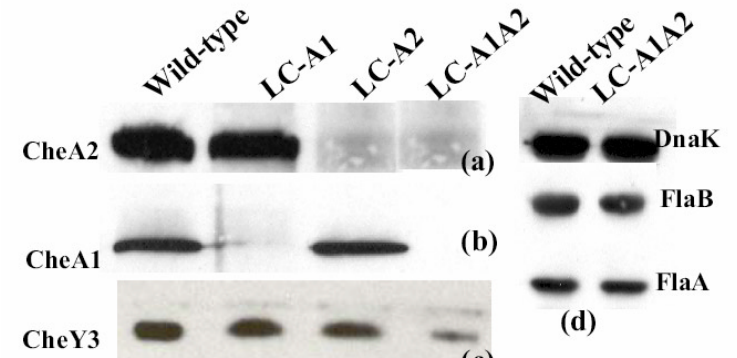

(c)
Figure 2.4 Western blot analysis using different antisera reacted against whole cell lysates of Wild Type and cheA mutants.

Lysate is noted across the top, antisera down the side. Anti-DnaK was used as an internal loading control. Down regulation of $\mathrm{CheY} 3$ in the double cheA1A2 mutant indicates there are polar effects on translation. FlaB is the major, and FlaA the minor periplasmic flagella filament proteins (21).

The reacting CheA2 protein migrated at approximately $98 \mathrm{kDa}$ on SDS-PAGE. In addition, a similar reaction was detected in lysates of LC-A1, but not in LC-A2 lysates. No reactivity was detected to a band corresponding to CheA1. These results indicate that CheA2 is expressed in wild-type cells, and that LC-A2 suffered a mutation in the cheA2 gene encoding that protein. To test for CheA1 expression, we raised an antiserum to recombinant CheA1. The resulting antiserum reacted strongly with purified recombinant CheA1, but not to recombinant CheA2 (not shown). Using this antiserum, we found strong reactivity when tested against both wild type and LC-A2 cell lysates at a band corresponding to CheA1 (79 kDa) (Figure 4b). No reactivity was detected in LCA1 lysates. These results indicate that both CheA1 and CheA2 were expressed in wild-type cells, and that mutants in the respective che $A$ genes were deficient in the proteins that these genes encode.

Several lines of evidence indicate that kan insertion into cheA1 or cheA2 did not markedly alter downstream transcription within their respective operons. For relative gene orientation please refer to Figure 2. First, RT-PCR analysis 
indicated all the genes downstream of both the cheA1::kan and cheA2::kan mutations were still transcribed (not shown). Second, although insertion of kan in cheA1 had no obvious phenotype (see below), mutations in the downstream gene orf569 resulted in cells that fail to reverse (Li and Charon, unpublished). Thus, the mutation in cheA1 did not negatively impact the expression of orf569. Third, because cheY3 is downstream of cheA2, we directly tested for CheY3 expression in mutant LC-A2 by Western analysis (Figure 2.4c). Using an antiserum directed to the recombinant protein, $\mathrm{CheY} 3$ was found to be expressed at approximately the same level in mutant LC-A2 as the wild type. In sum, results suggest that there was no inhibition of CheY3 synthesis by the kan insert.

Finally, mutations in cheX, which is immediately downstream of cheA2, result in cells that constantly flex (M. Motaleb and N. Charon, in preparation). Because the cheA2::kan mutant had such a markedly different phenotype than cheX::kan (see below), these results suggest that cheX is still expressed in the cheA::kan mutant. As a corollary, we tested whether transcription initiated at the flgB-kan promoter was responsible for transcription of the downstream genes by RT-PCR in mutant LC-A2.
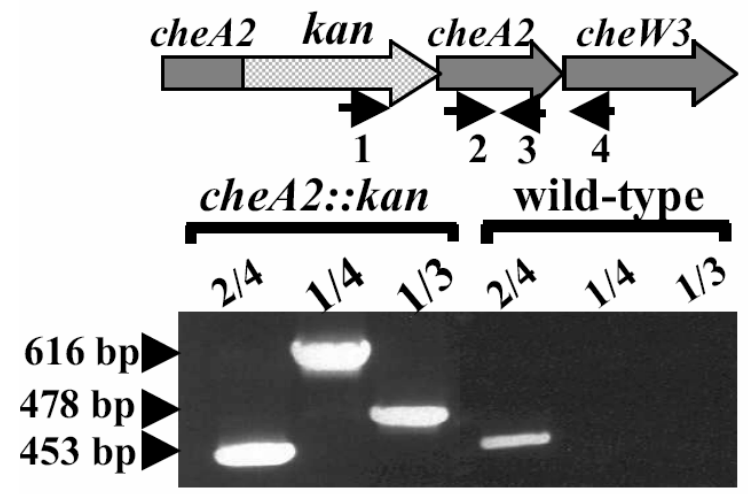

Figure 2.5 RT-PCR results indicating that transcription initiating within kan allows for downstream transcription of truncated cheA2 and cheW3.

Using primers complementary to kan and the downstream regions encoding the $\mathrm{C}$ terminal end of cheA2 or cheW3, we obtained products of the predicted size (Figure 2.5). These results suggest that transcription initiated within the kan gene 
is in part responsible for downstream transcription. The crux of these findings indicates that the observed phenotype is due to inactivation of the intended genes not polar effects. However to confirm these results complementation was performed as discussed in Chapter 3.

We analyzed the behavior of both LC-A1 and LC-A2 by tracking individual cells for at least one minute with the Hobson BacTracker. Cell velocity and reversal frequency in BSK II media with one percent methylcellulose were determined for the individual mutants and compared to the wild-type (91). The velocity of the wild type was found to be approximately the same as that of LC-A1 and LC-A2 (Table 2.2).

\begin{tabular}{lcc} 
Strains & $\begin{array}{c}\text { Reversal frequency, } \\
\text { reversals per min }\end{array}$ & $\begin{array}{c}\text { Velocity, } \\
\mu \mathrm{m} / \mathrm{sec}\end{array}$ \\
\hline Wild type & $18.89 \pm 5.08$ & $3.02 \pm 0.13$ \\
LC-A1 & $21.00 \pm 2.75$ & $2.48 \pm 0.71$ \\
LC-A2 & 0 & $3.01 \pm 0.61$ \\
LC-A1A2 & 0 & $3.10 \pm 0.32$ \\
\hline
\end{tabular}

Table 2.2 Reversal Frequency and Translational Velocity. for wild type, LC-A1, LC-A2, and LC-A1A2. Results suggest that LC-A1 retained a wild-type phenotype while mutants in che $A 2$ were not able to reverse. None of the $c h e A$ mutations affected swimming velocity.

These results indicate that mutations in cheA1 or cheA2 did not alter cell velocity. The reversal frequencies of the wild type and cheA1 mutant (LC-A1) cells were similar (18-21 reversals/min), but mutants in cheA2 (LC-A2 and LC-A1A2) swam in only one direction and failed to reverse. These mutants swam continuously with no stopping or flexing when tracked for as long as 5 minutes. These results indicate that inactivation of che $A 2$ markedly altered the reversal frequency, giving a similar phenotype as found in cheA mutants of externally flagellated bacterial species. 
Two different assays were used to test for chemotaxis in the wild-type and cheA mutants $(1,2,4,76)$. Rabbit serum, a required BSK II media component, was previously shown to be an attractant for $B$. burgdorferi (76). Using the capillary tube assay, wild type and LC-A1 had a strong chemotactic response to $0.5 \%$ rabbit serum (Figure 2.6a). In contrast, LC-A2 failed to have a response using this assay. Using the swarm plate assay, wild type and LC-A1 cells swarmed on soft agar plates in a ring-formation with BSK-II medium diluted 1:5 (Figure 2.6b). Swarms on undiluted medium were considerably smaller than those shown in Figure 2.6b. This observation suggests a steeper gradient of attractant formed in diluted medium during plate incubation. Because chemotaxis requires a gradient of attractant this finding is expected. The non-motile aflagellate flaB::kan mutant (MC-1) did not swarm.
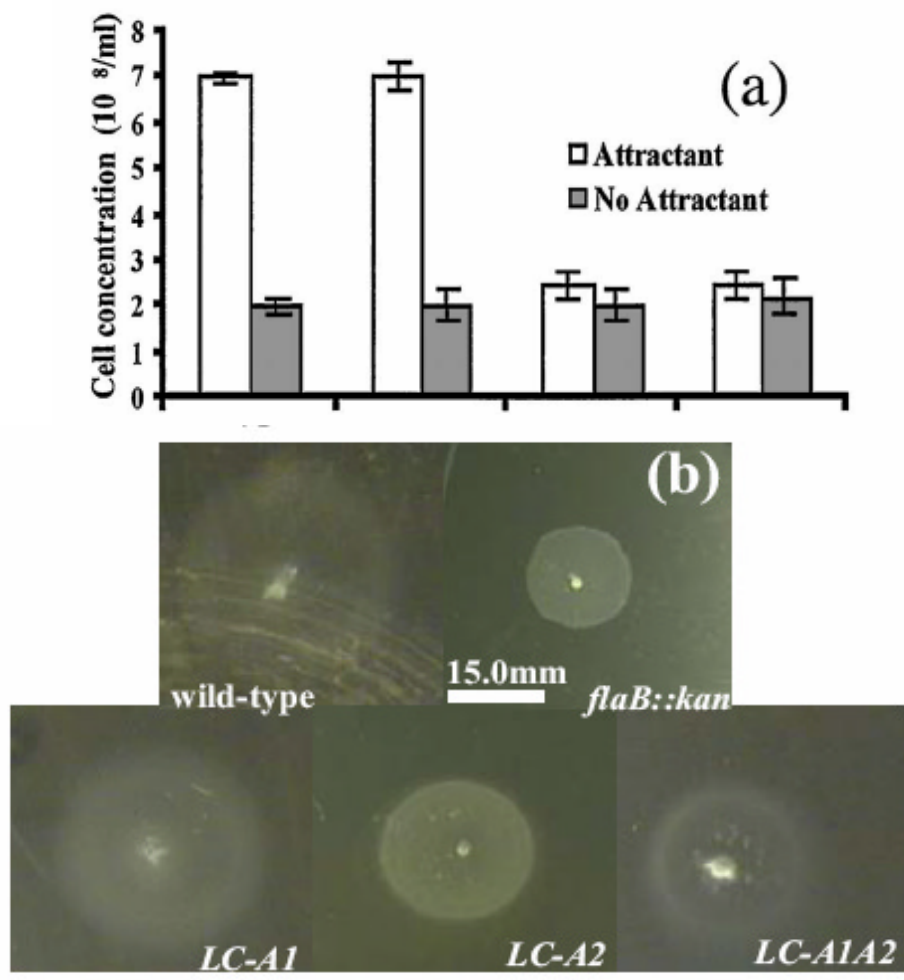

Figure 2.6: (a) Capillary tube (b), and swarm plate assays for chemotaxis of wild-type and mutants.

Capillary tubes contained

$0.5 \%$ rabbit serum, and swarm plate had BSK-II diluted 1:5.

LC-A1=cheA1::kan, LC-A2 = cheA2::kan, LC$\mathrm{A} 1 \mathrm{~A} 2=$ cheA1::kan cheA2::ermC, MC-1=flaB::kan.

Results indicate chemotactic ability follows: WT LC-A1 > LC-A2 LC-A1A2 > MC-1 
These results indicate swarm formation is motility dependent, and that the cells are chemotactic to components of the BSK-II medium. The LC-A2 mutant swarm is markedly smaller than the wild type. Results from both the capillary tube and swarm plate assays indicate that mutations in cheA2 but not che $A 1$ resulted in an inhibition of chemotaxis.

\section{Construction and analysis of a cheA1cheA2 double mutant. We were} intrigued that mutant LC-A2 swam only in one direction and failed to reverse. Mutations of $c h e A$ in other bacterial species result in the flagella rotating only in one direction. We would expect that analogous mutants of $B$. burgdorferi would result in cells constantly flexing rather than running (Figure 2.1). Because CheA1 was evident in LC-A2 (Figure 2.4b), its presence could conceivably influence flagellar rotation in the absence of CheA2. Accordingly, we constructed the double mutant cheA1cheA2 (LC-A1A2). Essentially the $1.3 \mathrm{~kb}$ kan cassette in pGA2kan was replaced by a $1.1 \mathrm{~kb}$ ermC cassette (221). After electroporation and selection with both wild type and LC-A1 as recipients, the resultant LC-A2e (cheA2::ermC) and LC-A1A2 mutants were characterized. As expected, LC-A2e was similar to LC-A2 as it failed to reverse (not shown). Western blot analysis verified that both CheA1 and CheA2 synthesis were inhibited in the LC-A1A2 mutant (Figure 2.4a,b). We tested for downstream effects of the erythromycin cassette in both LC-A2e and LC-A1A2. The expression of CheY3 was approximately one half that of the wild type in LC-A1A2 (Figure 2.4c) indicating that there was some downstream effect (Figure 2.2).

The swimming behavior of LC-A1A2 was compared to that of the wild type and the single mutants. This double mutant had a similar velocity to that of the wild type and single mutants, and it was identical to LC-A2 in its inability to reverse 
(Table 2.1). In addition, it was deficient in chemotaxis using both the capillary tube and swarm plate assays (Figure $2.6 \mathrm{a}, \mathrm{b}$ ). These results indicate that LCA1A2 resembles LC-A2 with respect to chemotaxis and cell reversal frequency. It also suggests that cells completely deficient in CheA swim in only one direction.

LC-A2 and LC-A1A2 periplasmic flagella analysis. Two possible explanations could account for the failure of LC-A2 and LC-A1A2 to reverse directions. One possibility is that both bundles of PFs constantly rotate but in opposite directions. Alternatively, the results could be explained by one bundle rotating in one direction with the other bundle non-existent or present but inactive. We tested for the presence of PFs at both cell ends. Electron microscopy revealed that bundles of PFs at both ends were present in LC-A2 and LC-A1A2 (not shown). In addition, Western analysis indicated that the quantity of flagellar proteins FlaA and FlaB in both mutants were approximately the same as the wild-type (Figure 2.4d). These results indicate that LC-A2 and LC-A1A2 have periplasmic flagella with similar protein content as the wild type.

Periplasmic flagella rotation was examined to determine if sets at both poles functioned. In other spirochete species, notably in those species with short periplasmic flagella that do not overlap, rotating periplasmic flagella influence the shape of the cell in the area where they reside. In addition, both ends simultaneously generate independent gyrational motion $(46,49,90)$. Because $B$. burgdorferi flagella overlap, independent motion of flagellar bundles is not as easily observed. The approach with $B$. burgdorferi was to analyze cells stuck to a glass surface near the center of the cell and video-record their motions (91). We analyzed these tethered cells that gyrated at the cell poles with little motion in 
the body of the cell. We found that in wild-type cells, changes in gyrational direction (i.e. CW, CCW) of one end relative to the other were readily apparent (not shown). Occasionally, the ends would alternate with one end stopping, and the other starting (Figure 2.7).

Wild-type
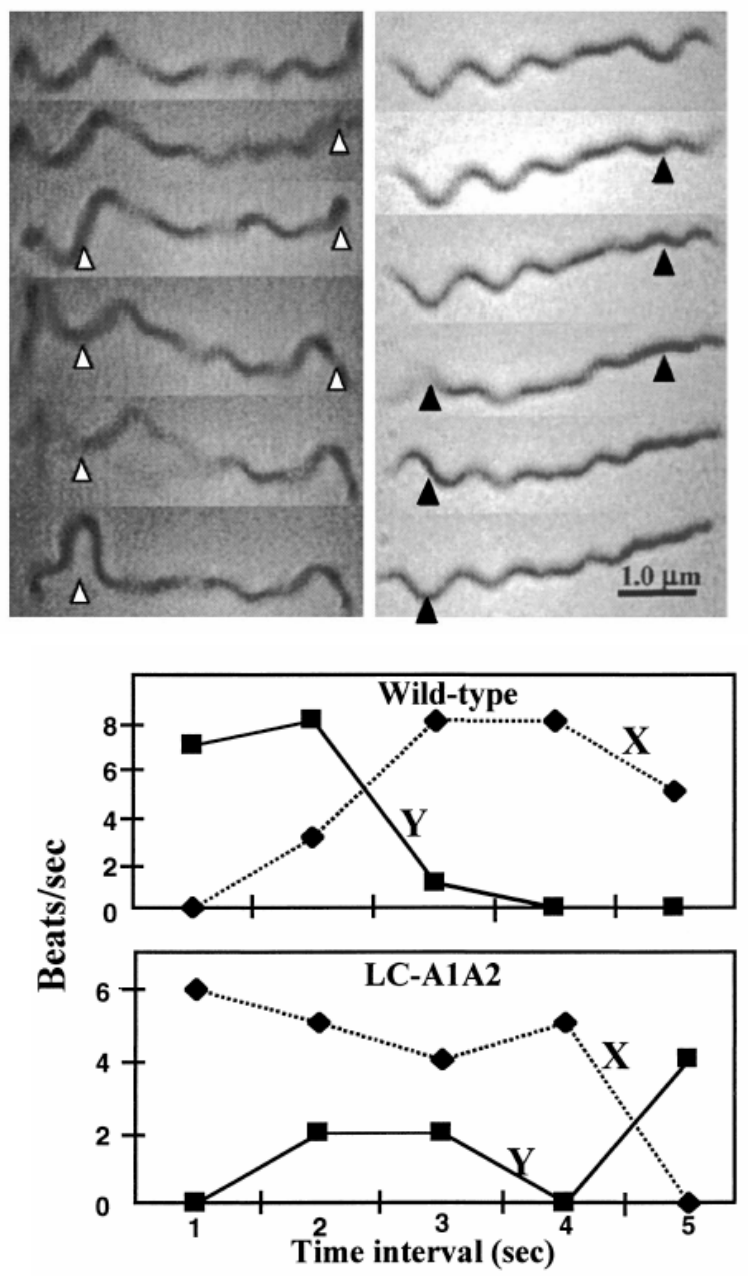

Figure 2.7: Sequential video frames of wild-type and LC-A1A2 mutant taken every 0.33 seconds.

Arrows points to the cell end having a change in position relative to the above frame. Data indicates that by frame 3 or 4 , both ends were able to change position independently of each other.

Beat frequencies (gyrations or beats/sec) of ends arbitrarily designated $X$ and $Y$ of WILD TYPE and the LC-A1A2 mutant are shown. The mean beat frequency was determined for each one second interval.

These results, and those previously described with respect to non-translatingflexing cells (91), suggest that the PFs at both cell poles are capable of rotation. Additionally, in cells with both ends simultaneously gyrating, the beat frequency at one end was occasionally seen to vary with that of the other end (Figure 2.7). Thus, in wild-type cells, our results indicate that both bundles of periplasmic flagella actively participate in cell motion. In analyzing mutants LC-A2 and LC- 
$\mathrm{A} 1 \mathrm{~A} 2$, we found that the cells did not change the direction of gyration of the ends as seen in the wild type. These results are consistent with the lack of reversal seen with free-swimming cells. As with the wild type, cells would occasionally alternate the gyration of their cell ends (Figure 2.7). In addition, the beat frequency at one end would sometimes vary with respect to the other end (Figure 2.7). These results indicate that both bundles of PFs in LC-A2 and LC-A1A2 participate in generating gyrational motion and can simultaneously rotate.

\section{Discussion}

In this communication we identified a fifth motility-associated promoter upstream of cheW2. Other characterized promoter sequences involved in motility include that of the $f l a B, f l a A, f l g B$, and flgK operons. All of these motility gene clusters are initiated by $\sigma^{70}$ promoters $(79,80)$; no motility gene specific promoters or sigma factors have yet to be identified in this species (76). To our knowledge, $B$. burgdorferi is the only bacterial species that lacks transcriptional cascade control of motility gene expression by alternative sigma factors. Note that the spirochetes S. aurantia, T. pallidum, T. denticola, Treponema phagedenis, and Brachyspira hyodysenteriae all have $\sigma^{28}$ specific motility promoters $(50,146)$. Several possible hypotheses could account for $B$. burgdorferi being unique. One possibility is that motility and chemotaxis are so vital for the survival of the spirochetes in both the tick and mammalian hosts that the genes involved are constitutively expressed $(79,146)$. In support of this hypothesis, B. burgdorferi expresses flaB message and produces PFs in both the tick and the mammalian host $(60,88)$. Alternatively, perhaps $B$. burgdorferi primarily relies on a translational control system recently described in other bacterial species $(5,77$, 120). Preliminary results with insertion mutants in flaA, flaB, flgE, and fliF support this possibility (M. Motaleb, M. Sal, and N. Charon, in press). For 
example, mutants in the hook gene flgE still synthesize $f l a B$ message but fail to synthesize detectable amounts of FlaB protein. These results differ from those of other bacteria, as mutations in flgE result in the inhibition of flagellin (FliC) mRNA and protein synthesis (52).

The two-cheA genes map in widely separated operons. Phylogenetic analysis indicates that cheA1, which is part of the cheW2 operon, likely evolved from the proteobacterial group. On the other hand, cheA2, which is part of the flaA operon, is most similar to che $A$ from other spirochetes. A similar conclusion was reached in analyzing the cheY2 and cheY3 genes (I. Zhulin, personal communication). In the other analyzed spirochete species, T. pallidum, T. denticola, only one cheA gene is present $(77,211,231)$. The cheW2 cluster could be a recent addition to the $B$. burgdorferi genome. Of the spirochete genomes sequenced, only $B$. burgdorferi resides in both arthropods and mammals, whereas T. pallidum, T. denticola dwell only in mammals. Perhaps the cheW2 cluster primarily functions within the tick, whereas the flaA gene cluster is most active within the mammalian hosts. The facultatively parasitic $L$. interrogans that infects amphibians, reptiles, and mammals has two cheA genes but expression of these genes awaits characterization (211).

LC-A2 and LC-A1A2 were found to be deficient in chemotaxis using both the capillary tube and swarm plate assays. These mutants swam only in one direction, and analysis of tethered cells indicated that both bundles of the PFs participate in translational motility; thus, the failure of these mutants to reverse is not the result of only one polar bundle of PFs being active. LC-A2 and LC-A1A2 mutants are similar to cheA mutants found in externally flagellated bacteria, in that they constantly run and do not reverse or tumble. However, in these other 
species, cheA mutants constantly rotate their flagella in one direction $(4,10,32$, 194, 264). In contrast, the bundles of PFs in B. burgdorferi would necessarily rotate in opposite directions for translation to occur (Figure 2.1). Thus, in the absence of CheA, i.e. the default state, one bundle of PFs rotates CCW, and the other bundle rotates $\mathrm{CW}$. These results imply asymmetry and thus structural differences with respect to the PFs at the opposite ends of the cell. Such flagellar asymmetry is not noted in other bacterial species. Similar results have been noted in cheA mutants of $T$. denticola consequently, it is likely that other spirochetes have asymmetrical rotation of the periplasmic flagella at opposite ends of the cells in the absence of CheA (156).

Asymmetrical location of cellular structures is described for several bacterial species $(158,232)$. For example, the stalk structure localizes at one end of the cell in Caulobacter crescentus at a site previously occupied by the flagellum. The ActA protein of Listeria monocytogenes, and the IcsA protein of Shigella flexneri localize at one of the cell poles in each of these species. Localization of these proteins targets the old cell pole, but their mechanisms of localization are different. In S. flexneri, localization of IcsA is dependent on direct targeting to that specific pole, whereas ActA seems to be excluded from the newly synthesized cell pole. Perhaps in B. burgdorferi, there is association with an unknown factor associated with the flagellar switch complexes at one cell pole. This association could result in the periplasmic flagella at that end rotating $\mathrm{CW}$ rather than $\mathrm{CCW}$ in the default state. Now that the genetic tools are available for B. burgdorferi, the major factors for this asymmetrical rotation in spirochetes can begin to be deciphered. 
We thank S. Goldstein, M. Sal and D. Yelton for helpful discussions, and A. Barbour, J. Benach, B. Johnson, and P. Matsumura for antibodies. We also thank R. Lux, W. Shi, and I. Zhulin for stimulating discussions and communicating unpublished material. Public Health Service Grants Al29743 to N.W.C., Al 43063 to F.C.C., and the WVU MD/PhD program to R.G.B, supported this research. 
In preparation for Applied and Environmental Microbiology:

\section{Chapter 3: Identification of specific chemoattractants for Borrelia}

burgdorferi: A flow cytometric based chemotaxis assay.

RICHARD G. BAKKER, CHUNHAO LI, MICHAEL MILLER, CYNTHIA

CUNNINGHAM, AND NYLES W. CHARON*

Department of Microbiology and Immunology, Robert C. Byrd Health Sciences

Center,

West Virginia University, Morgantown, West Virginia 26506-9177

Running title: Borrelia burgdorferi

Key Words (Borrelia burgdorferil chemotaxis/histidine kinase

CheA/complementation)

* Corresponding author. Mailing address, Department of Microbiology, Immunology and Cell Biology, West Virginia University, Box 9177, Robert C.

Byrd Health Sciences Center, Morgantown, West Virginia 26506-9177. Phone: (304) 293-4170. Fax (304) 293-7823.

Electronic mail address: ncharon@hsc.wvu.edu

Section: genetics 


\section{Abstract}

Measuring the chemotactic response in Borrelia burgdorferi, the Lyme disease spirochete, was previously difficult because chemotaxis assays were suboptimal, no defined attractants were known, and enumeration was slow, laborious, and ineffectual at low cell concentrations. We overcame these limitations by developing a protocol for enumerating cells by flow cytometry. Once this enumeration method was validated with direct comparisons to PetroffHausser counting data, we were able to screen for attractants using a modified capillary tube assay. N-acetyl-glucosamine and chitosan dimers were found to be chemoattractants. Using flow cytometric enumeration and $\mathrm{N}$-acetylglucosamine, the capillary tube assay was optimized with respect to cell concentration, incubation time, chamber size, viscosity, motility buffer preparation, culture density, and cell starvation. The optimized assay was then used to test the hypothesis that compounds that are known nutritional requirements, or have putative transporters encoded in the genome, will act as chemoattractants. The screen for chemoattractants, to date, have included serine, glycine, N-n-diacetyl-chitobiose, glucose, glutamate, putricine, spermidine, and glucosamine, the latter four yielding a positive chemotactic response. Previously, rabbit serum and tick saliva were shown to be chemoattractants. However, these are complex biological mixtures with unknown and likely variable contents limiting their utility for the study of chemotaxis. Chemoattractants yield no response from cheA2 mutants while the complemented form behaved similarly to wild type. In sum, this is the first report of defined chemoattractants for $B$. burgdorferi and enumeration of a spirochete species by flow cytometry. Protocols developed for this work will hopefully facilitate measuring the chemotactic response in other slow growing prokaryotic species. 


\section{Introduction}

Chemotaxis and motility are putative spirochete virulence factors $(47,154)$. B. burgdorferi, the causative agent of Lyme disease, uses a flat wave swimming modality that allows these bacteria to translate in viscous gel-like media that slow or stop most externally flagellated bacteria (47). Several observations suggest motility and chemotaxis are vital to Borrelia burgdorferi cell ecology. As discussed in Introduction Section E, the B. burgdorferi life cycle involves transmission from an arthropod vector to a mammal and back to the arthropod over the course of several seasons (249). When an infected Ixodes tick feeds on a mammal, $B$. burgdorferi in the tick translocate from the gut, to the salivary glands and then into the host (193). A season later, when the next generation of Ixodes nymphs feed and become infected, $B$. burgdorferi likely using chemotaxis and motility concentrate at the site of feeding and is pulled back into the arthropod with the blood meal $(143,204,289)$. Further evidence for the role of chemotaxis and motility in pathogenesis comes from clinical experience. The clinical course of Lyme disease includes spreading annular erythematous lesions, thought to be the result of bacterial movement through the dermis away from the site of the arthropod bite $(179,249,251)$. Bacteria from these lesions then spread hematogenously and across facial planes to infect other organs. In sum, the clinical course and movements in nature likely require a robust chemotaxis and motility system.

There is no direct evidence that chemotaxis and/or motility play a role in $B$. burgdorferi pathogenesis. An undefined flagellar-less mutant demonstrated diminished capacity to invade endothelial cells. It also was non-infectious, surviving only 24 hours post intradermal inoculation (219). The mutation in this strain remains undefined and its plasmid content was never characterized. The latter point is significant because plasmid loss occurs readily during laboratory passage and correlates to a dramatic loss of virulence (135). This gap in the 
literature promises to be filled by genetic advances that allow manipulation of pathogenic strains. Thus data, while not conclusive, do infer that chemotaxis and motility are intrinsic to the pathogenesis of $B$. burgdorferi.

Chemotaxis and motility are best understood in the peritrichous enteric species Escherichia coli and Salmonella enterica serovar Typhimurium (hereafter referred to as S. enterica) (10). Bacterial chemotaxis, as described in E. coli and $S$. enterica, involves a sensory transduction system that enables cells to respond to environmental stimuli whereby cells swim toward a favorable medium, or away from one that is less favorable. A two-component regulatory system involving a histidine kinase and response regulator play an essential role in this response. Methyl accepting chemotaxis proteins (Mcps) bind attractant molecules in the periplasmic space leading to CheR mediated methylation. Methylation occurs concomitantly with a reduction in autophosphorylation of the associated histidine kinase CheA. Activated CheA phosphorylates the response regulator CheY. Phosphorylated CheY (CheY-P) then interacts with the switch complex at the flagellar motor to change direction of flagellar rotation from the default counterclockwise $(\mathrm{CCW})$ to clockwise $(\mathrm{CW})$ as viewed along the flagellum toward the motor insertion. These modes of rotation correlate with running or tumbling, respectively. Mutants in $\mathrm{CheA}$ or CheY exhibit a constantly running phenotype. This system allows organisms to swim up a gradient attractant or away from a toxic environment in a manner known as a biased random walk. The suppression of CheA autophosphorylation activity is transient. As the methylation sites on the Mcps become full, the suppression of CheA autophosphorylation activity ceases. This process is known as adaptation, and allows for taxis to additional attractants on the background of a first attractant $(10,32)$.

B. burgdorferi motility differs from the E. coli - S. enterica model structurally and genetically. Structurally, spirochete flagella bundles are sandwiched between the cell cylinder and outer membrane sheath with motor clusters positioned 
subterminally at each pole, earning them the name periplasmic flagella (PFs). This structure contrasts with most motile bacteria species, which are externally flagellated. The mechanism of rotation appears to be largely homologous between internally and externally flagellated bacteria. As mentioned, CCW rotation in externally flagellated bacteria leads to smooth translation. In contrast, spirochetes must have their polar flagella rotate in opposite directions, as observed from the center of the cell, for smooth translation to occur $(47,91)$.

Genetic analysis indicates B. burgdorferi differs from the E. coli-S. enterica paradigm and suggests the importance of chemotaxis and motility to the organism's ecology. B. burgdorferi has a genome a quarter the size of $E$. coli, but carries not only the full complement, but also multiple copies of several chemotaxis and motility genes. These motility and chemotaxis genes constitute approximately $5 \%$ of $B$. burgdorferi's genetic capacity. Additionally, these putative chemotaxis and motility genes are expressed in the absence of the sigma factor dependant cascade control used in the E. coli-S. enterica model and are constitutively expressed in the arthropod and mammalian hosts $(6,45,76$, $79,82)$. These differences, and the large percentage of the $B$. burgdorferi genome dedicated to these functions, highlight the importance of motility and chemotaxis to the $B$. burgdorferi life cycle (251).

Measuring bacterial chemotaxis with the capillary tube assay is well documented in the literature $(2,4,95,126,177,189)$. The standard assay is to place a putative attractant in a capillary tube that is then incubated with one orifice submerged in a cell suspension. Cells entering the tube during incubation are then enumerated by viable counts. Several optimization steps were performed to adapt this assay to $B$. burgdorferi. The slow growing nature of $B$. burgdorferi makes measuring the chemotactic response with viable counts impractical because colony formation requires approximately 20 days. Initially, assaying chemotaxis in $B$. burgdorferi required enumeration using a Petroff-Hausser counting chamber. This technique requires high cell densities and is labor 
intensive. Here we overcame these limitations by using flow cytometry for enumeration.

Characterizing defined attractants is critical to describing the role of chemotaxis signal transduction components. Previously documented B. burgdorferi attractants, rabbit serum and tick saliva, are complex biological mixtures making it difficult to determine which compounds have biological activity and which are inert $(234,236)$. In other bacterial species, attractants are often used for nutrition $(3,29,128,136,155)$. With the availability of the $B$. burgdorferi genome, several putative transporters were identified $(45,76)$. This knowledge of transporters has lead to the characterization of the transport mechanism for the known nutrient and chemoattractant $\mathrm{N}$-acetyl-glucosamine (273). Our hypothesis states selected compounds with transporters encoded in the $B$. burgdorferi genome, as well as known nutrients, will act as chemoattractants. Accordingly, several of these compounds were assayed using the capillary tube methodology.

\section{Materials and Methods}

Bacterial strains and growth conditions. A single clone of high-passage $B$. burgdorferi B31 strain and its cheA2 null mutant cheA2::kan (referred to as B31A and LC-A2, respectively) were grown at $34^{\circ} \mathrm{C}$ in liquid BSK-II medium supplemented with $5 \%$ rabbit sera $(17,70,144)$. Kanamycin $(100-350 \mu \mathrm{g} / \mathrm{ml})$ and streptomycin $(80 \mu \mathrm{g} / \mathrm{ml})$ were used for selection as needed (144). The preparation of $B$. burgdorferi competent cells and the electro-transformation and selection procedures were previously described $(176,220)$.

Capillary Tube Chemotaxis Assay. The capillary tube assay as developed by Pfeiffer and described by Adler was optimized to measure $B$. burgdorferi chemotaxis (1). B. burgdorferi were grown to late log phase $\left(\sim 7.5 \times 10^{7}\right.$ cells $\left./ \mathrm{ml}\right)$ from an initial incoulum of $2 \times 10^{5} \mathrm{cells} / \mathrm{ml}$ and centrifuged at room temperature for $8 \mathrm{~min}$ at $1800 \mathrm{xg}$ and re-suspended in motility buffer. Motility buffer 
consisted of phosphate buffered saline (PBS, $\mathrm{KCl} 2.68 \mathrm{mM} ; \mathrm{KH}_{2} \mathrm{PO}_{4} 1.47 \mathrm{mM}$; $\mathrm{NaCl} 136.9 \mathrm{mM}$; and $\mathrm{Na}_{2} \mathrm{HPO}_{4} 8.10 \mathrm{mM}$ ) at pH 7.4 supplemented with $2 \%$ recrystallized bovine serum albumin (BSA) (Sigma-Aldrich Co, St. Louis, MO USA) and $1 \times 10^{-4} \mathrm{M}$ ethylenediamine-tetraacetic acid (EDTA) (233). Chemotaxis chambers consisted of a $2 \mathrm{ml}$ microfuge tube with a Parafilm sheet closed under a perforated cap. After gentle re-suspension in motility buffer, the cells were enumerated by flow cytometry, diluted to $1 \times 10^{7}$ cells $/ \mathrm{ml}$ in equal parts motility buffer and $2 \%$ methylcellulose (400Cpi Sigma-Aldrich Co., St. Louis, MO, USA) in PBS and $300 \mu \mathrm{l}$ pipetted into the microfuge tubes. Attractant filled $70 \mu \mathrm{l}$ capillary tubes (cat \# 22-362-574 Fisher Scientific, Pittsburgh, PA, USA), plugged with silicone grease, were inserted into the chambers. Attractant solutions had the same methylcellulose and BSA concentrations as the cell pools. Assay viscosity was measured with a number 100 Cannon-Fenske routine viscometer and was determined to be $224 \mathrm{Cpi}$ at $33^{\circ} \mathrm{C}$. The chambers were then incubated horizontally at $33^{\circ} \mathrm{C}$ in $3 \% \mathrm{CO}_{2}$ for 120 minutes. After incubation, the capillary tubes were removed, wiped with a paper towel, and the contents collected by centrifugation at $1000 \mathrm{xg}$ for less than 5 seconds. Flow cytometry was then used to enumerate the spirochetes. Optimization experiments are presented in the Results section.

Three controls were used to test that chemotaxis was measured in our system. First, to assay background migration, buffer filled (no attractant) tubes tested random accumulation of cells in capillary tubes (1). Second, the non-chemotactic cheA2 mutant (LC-A2) that was previously shown by swarm plate and capillary tube assay not to respond to rabbit serum was used as a negative control (144). Finally, assays with no gradient of attractant were tested. This control consisted of an attractant solution placed in the cell pool as well as in the capillary tube. In the no gradient situation, cells should not demonstrate enhanced migration into the capillary tube compared to the buffer control (10 and references within). The chemotactic response was expressed as factor increase over buffer control. A 
response greater than 2 times the buffer control was considered significant (59, $124,126,168,177)$. The response was determined by:

(1) Calculating the mean number cells $\left(\sim 1 \times 10^{5} \rightarrow 1 \times 10^{6} \mathrm{cells} / \mathrm{ml}\right)$ in buffer filled tubes $(n=5)$;

(2) Dividing number of cells in an experimental capillary tube $(n=5)$ by the average number in buffer filled tubes $(n=5)$;

(3) Calculate the mean and standard deviation (SD) of the values arrived at in (2). Results are reported as mean $\pm S D$.

All chemicals were obtained from Sigma-Aldrich Chemical Co. St. Louis, MO, USA, with the exception of chitosan dimers (glucosamine dimers) (USBiologcial Co., Swampscott, MA, USA) and N-n-diacetyl-chitobiose (Cape Cod Associates Inc., East Falmouth, MA, USA)

Flow Cytometric Enumeration of $\boldsymbol{B}$. burgdorferi. Samples were prepared by diluting cell suspensions in $0.01 \mathrm{M} \mathrm{HEPES}, 0.15 \mathrm{M} \mathrm{NaCl}, \mathrm{pH} 7.4$, containing 10 $\eta \mathrm{M}$ fresh Syto61 (Molecular Probes Inc., Eugene, OR, USA) and $3 \rightarrow 5 \times 10^{3} 6$ $\mu \mathrm{m}$-diameter quantitative polystyrene beads $/ \mathrm{ml}$ (catalog \# 4k-06 Duke Scientific Co., Palo Alto, CA, USA). All media and solutions were $0.1 \mu \mathrm{m}$ filtered before use. Syto61 is a membrane permeable nucleic acid dye giving a fluorescent signal readable on channel FL4 (139). Bead enumeration acted as an internal control to insure the volume enumerated was consistent for each sample. Samples were run at a rate of 12 or $16 \mu \mathrm{l} /$ minute for 60 or 120 seconds. This run time yielded an event count of at least 300 in the $\mathrm{R} 2$ region where cells sorted. A Becton-Dickinson FACScalibur with $15 \mathrm{~mW}$ air-cooled argon and red diode lasers operating at $488 \eta \mathrm{M}$ and $635 \eta \mathrm{M}$, respectively was used for all counting procedures. No compensation for spectral overlap was necessary, as there was no spectral overlap between detectors (42). Representative machine settings can be found in Table 3.1. All data acquisition and analysis were done with CellQuestPro (Becton-Dickinson\&Co., San Jose, CA, USA) and MSExcel (Microsoft Co., Redman, WA, USA). 


\begin{tabular}{llllll} 
Parameter & Detector & Voltage & AmpGain & Mode & Use \\
\hline P1 & FSC & E01 & 1.00 & Log & \\
P2 & SSC & 334 & 1.00 & Log & \\
P3 & FL1 & 487 & 1.00 & Log & GFP \\
P7 & FL4 & 640 & 1.00 & Log & Syto-61
\end{tabular}

Table 3.1 Representative FACScalibur Machine Settings.

Slight adjustments were made based on variations in experimental conditions. No compensation was necessary.

Motion Analysis and Swarm Plates. Two methods were used to track swimming cells. First, the Hobson BacTracker was used to track the motion of an individual $B$. burgdorferi cell to determine its reversal frequency and velocity as described before (144). Second, a software package marketed as Volocity (Improvision Inc, Coventry, UK ) proved able to track B. burgdorferi more proficiently. Film of swimming cells was captured with iMovie on a PowerMac Dual G4 (Apple Computer Inc., Cupertino, CA, USA) using a Scion LG-5 (Scion Inc, Fredrick, MD, USA) frame grabber card and a Dage MTI (Dage-MTI Inc. Michigan City, IN, USA) black and white video camera. This video was exported as a QuickTime (Apple Computer Inc. Cupertino, CA, USA) movie, and imported into OpenLab (Improvision Inc, Coventry, UK) where the frames were cropped, calibrated using a stage micrometer, and saved as a .LIFF file. Volocity was then used to analyze video sequences on a PowerMac Dual G5 (Apple Computer Inc., Cupertino, CA, USA). Cells were prepared as if they were to be used in a chemotaxis assay, centrifuged, resuspended in motility buffer and methylcellulose, and then visualized at $200 \times$ magnification at $35^{\circ} \mathrm{C}$ with a heated stage (Physitemp Inc, Clifton, NJ, USA) equipped Zeiss Axioskop 2 (Carl Zeiss Inc. Jena, Germany). Swarm plate assays were performed as previously described (144). 


\section{Results}

Use and optimization of flow cytometry to enumerate cells from the capillary tube assay. Previously, Petroff-Hausser counting was used to enumerate $B$. burgdorferi in chemotaxis assays $(144,233)$. This procedure is cumbersome, requires high cell densities making interpretation difficult, and is labor intensive. Therefore, a flow cytometry protocol was developed to enumerate $B$. burgdorferi. Flow cytometry allows for visualization of a cell population based on scattered and transmitted laser light. The simplest form shows particles on an $\mathrm{XY}$ axis, with the signal from scattered light (side scatter) plotted the $\mathrm{Y}$-axis, and transmitted light (forward scatter) on the $\mathrm{X}$-axis. By using side scatter detectors that sense specific wavelengths of light, the presence of a fluorescent dye is readily visualized. We used this capability to read signal from Syto-61, which is a membrane permeable nucleic acid dye. By staining with Syto-61, we were able to differentiate which particles have nucleic acids from those that do not. As expected, nucleic acid containing particles were cells that are then easily counted, or in the parlance of flow cytometry, "gated on" (61).

Figure 3.1 shows four representative flow cytometric analysis dot plots with identical gating. Forward scatter is represented on the $\mathrm{X}$-axis and correlates with particle size. Side scatter is represented on the $\mathrm{Y}$-axis and correlates with cellular complexity (Plot A) or fluorescence (Plots B, C, and D). Plots A and B demonstrate the presence of $B$. burgdorferi in an 


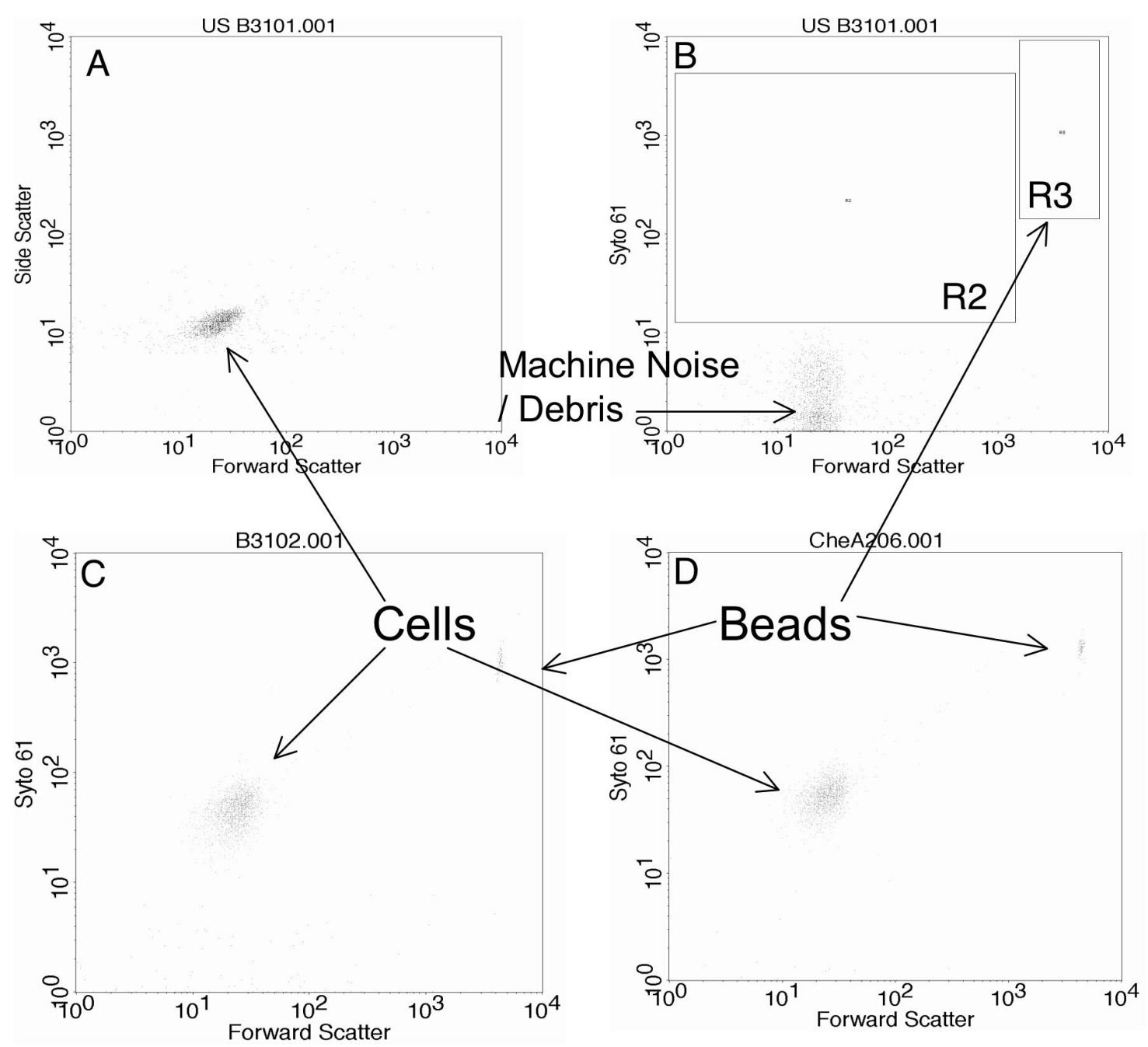

Figure 3.1 Four representative dot plots with identical gating.

Forward scatter is represented on the $\mathrm{X}$ axis for all plots and side scatter (cellular complexity) (Plot A) or intensity of Syto61 staining (Plots B, C, D) are shown on the $Y$-axis. Plots $A$ and $B$ show unstained wild type B31 cells with side scatter or emissions read on FLH-4 (Syto61), respectively. As expected, unstained cells are not visualized with the detector for Syto61 (plot B). Plots $C$ and D show populations of stained B31 and cheA2 mutants in Region 2 (R2). Beads (R3) can also be seen forming a distinct population.

unstained sample. Plot A depicts the physical complexity of a cell population by graphing non-fluorescent events. Results indicate, as expected, particles (cells) cluster based on size (forward scatter plots $A, C$, and D Figure 3.1). Plot $B$ illustrates that unstained cells give no signal when measured with a fluorescence detector, showing there is no cellular autofluorescence to skew cell counts. Plots 
$\mathrm{C}$ and $\mathrm{D}$ illustrate that the addition of dye allows for segregation of cells and debris. Plots $C$ and $D$ show that populations of stained B31 and LC-A2 fell into the region (gate) 2 and the $6 \mu \mathrm{M}$ diameter polystyrene beads into region (gate) 3 (R3). These parameters allowed for the delineation of $B$. burgdorferi from beads, background debris, and machine noise based on size difference and staining characteristics, leaving little concern that non-staining particulate debris lead to skewed cell counts.

Flow cytometry provided the tools to overcome the limitations to the capillary tube chemotaxis assay as outlined in the Introduction. Flow cytometry can enumerate other bacterial species, but this is the first report of enumeration of a spirochete $(42,98,139,243,270)$.

FACS vs. P-H

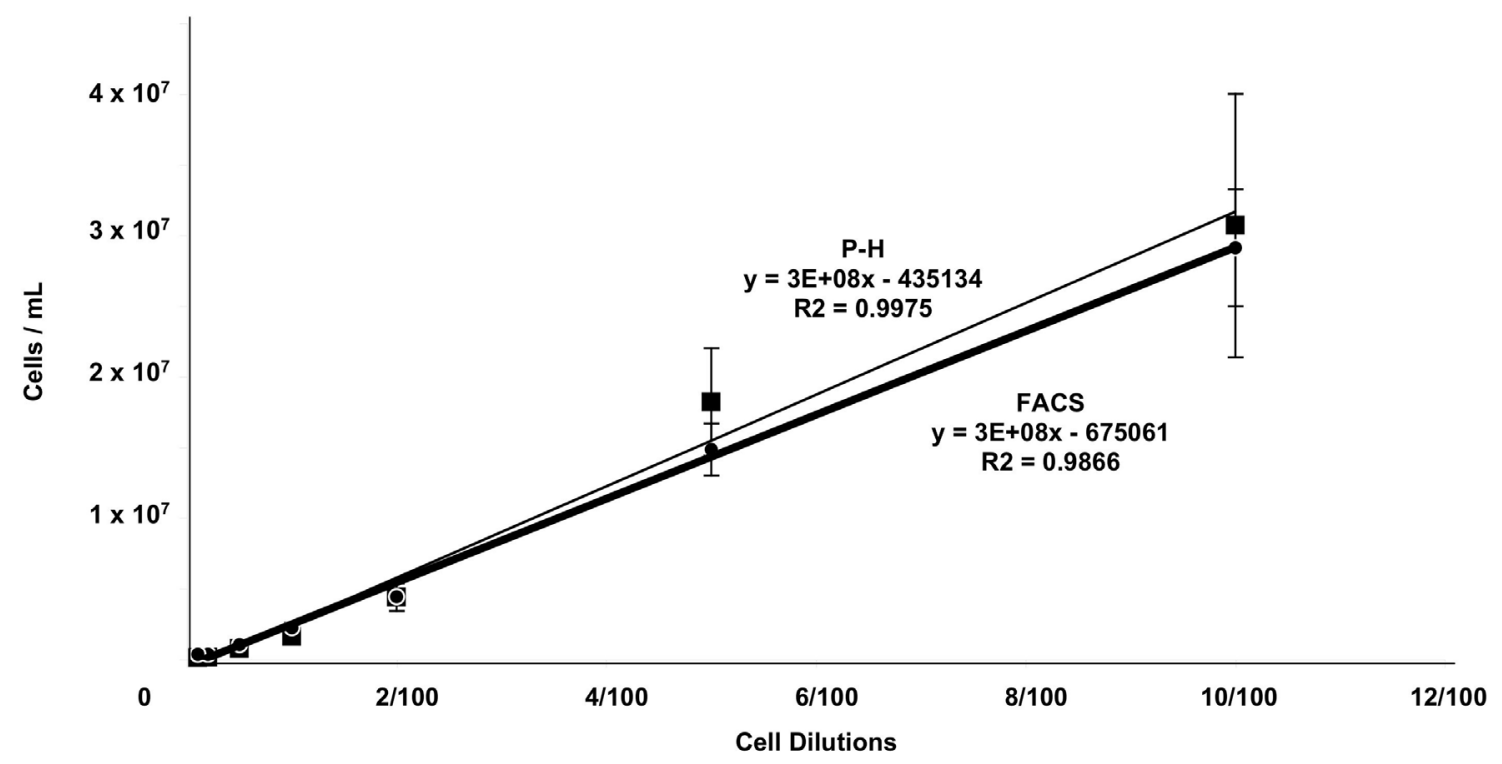

Figure 3.2: Flow cytometry (FACS) vs. Petroff Hausser Counting. Across a range of dilutions both counting methods gave similar results with correlation coefficients of $>0.95$. The equations of the linear regression lines are noted as well. Cells were centrifuged, suspended in motility buffer and then diluted as needed in PBS for enumeration by Petroff-Hausser $(\mathrm{P}-\mathrm{H})$ counting (300 to 600 cells / field) (squares). Alternatively the same samples counted by PH were diluted 1:500 in HEPES + Syto61 dye and counted by flow cytometry (circles). 
The validity of the flow cytometric approach is demonstrated in Figure 3.2, which directly compares $B$. burgdorferi cultures counted with flow cytometry (FACS) or a Petroff-Hausser counting chamber. Results from either counting technique were statistically identical, and both had correlation coefficients of $>0.95$. The equations of linear regression lines are provided. Because results derived using flow cytometry greatly increased the productivity of the capillary tube assay, it was adopted as the primary counting method. Additionally, it allowed for enumeration of lower densities of cells. This capability led us to use $1 \times 10^{7}$ cells per $\mathrm{ml}$ in an assay instead of the approximately $1 \times 10^{9}$ cells per $\mathrm{ml}$ used previously (144). Using lower densities of cells decreases the possibility taxis toward a metabolite generated during incubation occurs and minimizes cell clumping.

Optimization of parameters for the capillary tube assay. Once the flow cytometry protocol was generating data, we used the high throughput of this system to optimize the classic capillary tube assay for $B$. burgdorferi. The capillary tube assay is predicated on bacteria swimming up an attractant gradient generated around the mouth of a capillary tube. To optimize this assay, seven variables were adapted. These included: cell concentration, assay incubation time, assay chamber, viscosity of the assay media, bovine serum albumin preparation used in the motility buffer, phase of culture growth, and starvation of cells in motility buffer before exposure to the attractant.

a) Cell concentration assays using different concentrations of cells in the cell pool indicated that low concentrations of cells $\left(1 \times 10^{5} \mathrm{cells} / \mathrm{ml}\right)$ gave a stronger response to lower concentrations of attractants $(0.01 \mathrm{M} \mathrm{NaG})$ while high concentrations of cells $\left(1 \times 10^{10}\right.$ cells $\left./ \mathrm{ml}\right)$ responded better to high concentrations of attractant $(0.1 \mathrm{M} \mathrm{NaG})$. At high cell densities low concentrations of attractant were perhaps quickly metabolized, so less attractant would be available to trigger a chemotactic response. All subsequent experimental assays used $1 \times 10^{7} \mathrm{cells} / \mathrm{ml}$. This concentration 
gave sufficient cell numbers for reliable enumeration, were consistent with prior work, and limited the possibility that a metabolite could be acting as an attractant (Figure 3.3) $(1,59,177)$.

\section{Chemotactic Response to $0.01 \mathrm{M}$ or $0.1 \mathrm{M} \mathrm{N}$-acetyl-glucosamine as a}

Fucntion of Cell Density

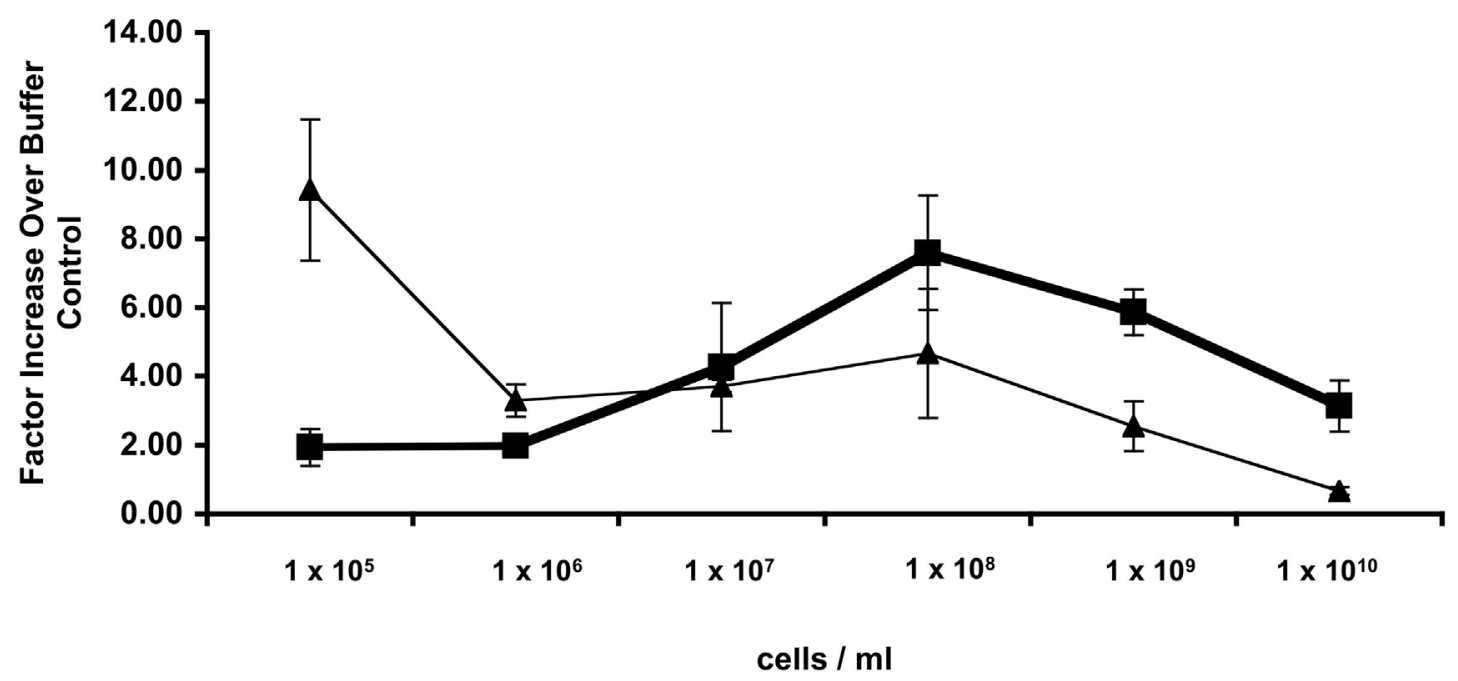

Figure 3.3 The chemotactic response as a function of the number of cells in the cell pool.

The thin line illustrates the response to $0.01 \mathrm{M}$ while the heavy line illustrates the response to $0.1 \mathrm{M} \mathrm{N}$-acetyl-glucosamine, respectively.

b) Incubation time was determined by counting cells entering attractant filled tubes at 30-minute intervals for a 4 -hour period. Cell numbers increased for 90 to 120 minutes (Figure 3.4). The chemotactic response likely fell after this point due to nutrient limitation. Therefore, 120 minutes was used as the optimal assay time. 


\section{Chemotactic Response per Assay Time}

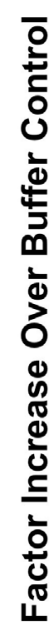

0.00

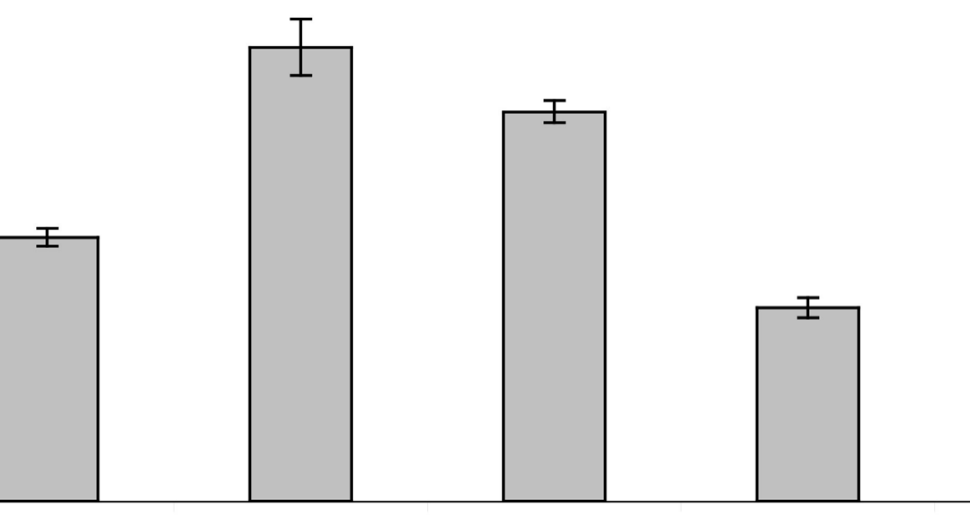

60 minutes

90 minutes

120 minutes 180 minutes

240 minutes

Figure 3.4: Chemotactic response per assay time.

These experiments were done as 5 separate assays from the same cell culture. In these experiments, assays were ended every 30 minutes to measure the chemotactic response over time to $0.01 \mathrm{M} \mathrm{N}$-acetyl-glucosamine. Based on experience and results similar to those shown we chose 120 minutes as the standard assay time.

c) A new assay chamber was sought because glass $U$ tubes were cumbersome for performing multiple assays. A $2 \mathrm{~mL}$ centrifuge tube was used in place of Adler's U-tube-on-a-plate design to create the chambers. The centrifuge tube has the advantages of being stackable, creating a uniform pool size, and limiting investigator exposure to $B$. burgdorferi contaminated glass. The latter is an important consideration if the chemotactic response of pathogenic strains are to be assayed. Attractantfilled $70 \mu \mathrm{l}$ micro-hematocrit capillary tubes provide the volume necessary for flow cytometric analysis. Consistently, the centrifuge tubes were more proficient and economical than the $U$ tubes (Figure 3.5). 
U Tube vs. $2 m L$ Tube

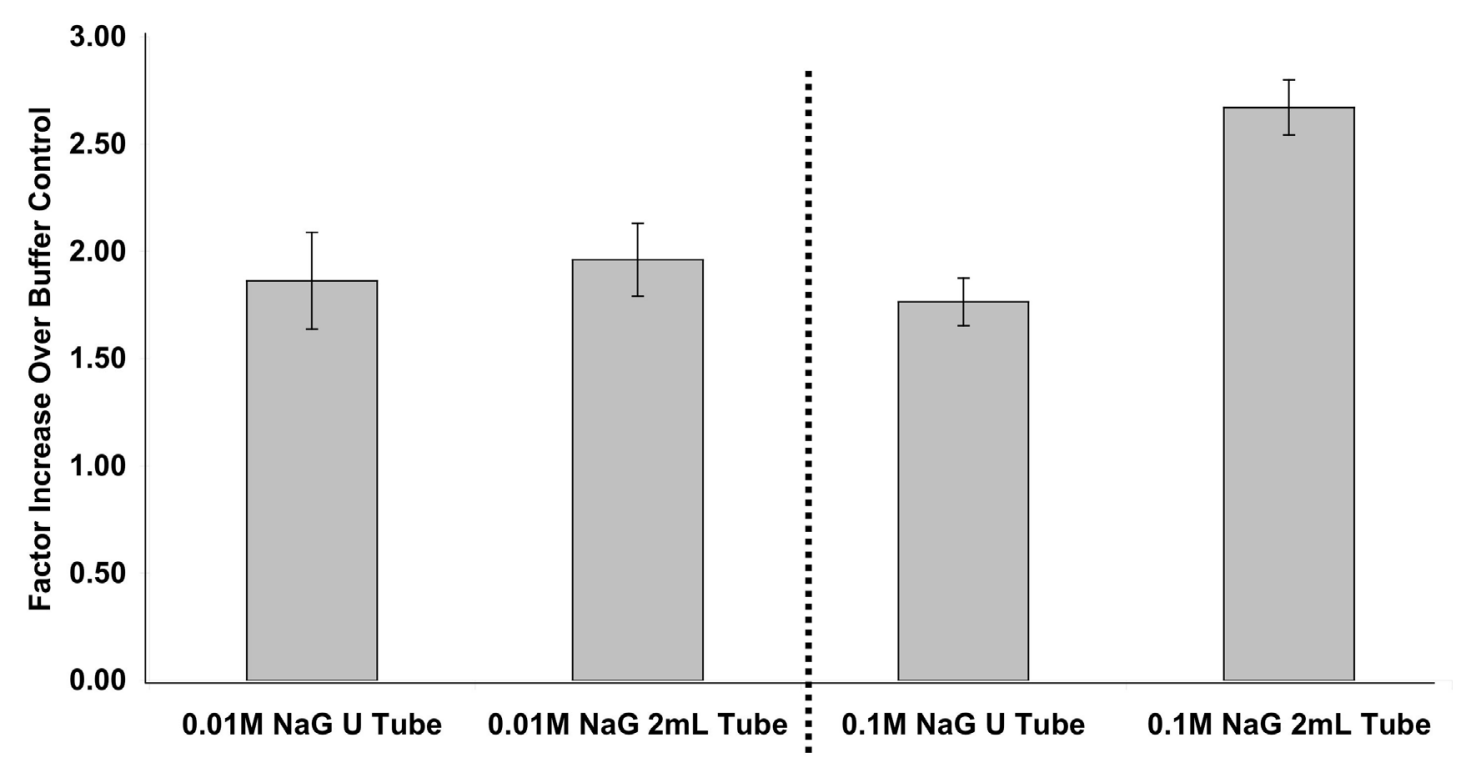

Figure 3.5: $U$ Tube vs. $2 \mathrm{ml}$ microfuge tube assay for measuring chemotaxis.

$\mathrm{N}$-acetyl-glucosamine $(\mathrm{NaG})$ was used as the attractant in $0.01 \mathrm{M}$ or $0.1 \mathrm{M}$ solutions. The $2 \mathrm{ml}$ microfuge tubes consistently gave more accurate and economical results.

d) Macroscopic viscosity is known to increase the translational velocity of spirochetes $(90,96,131,218)$. Increasing viscosity facilitates the entry of spirochetes into attractant filled tubes while slowing non-specific entry of cells into buffer filled tubes. We found the added methylcellulose reduced variability between tubes and was adopted as standard procedure (Figure 3.6). The addition of methylcellulose in the assay media is a well documented method to increase the macroscopic viscosity (25). 


\section{Addition of Methylcellulose to Assay Increases Response}

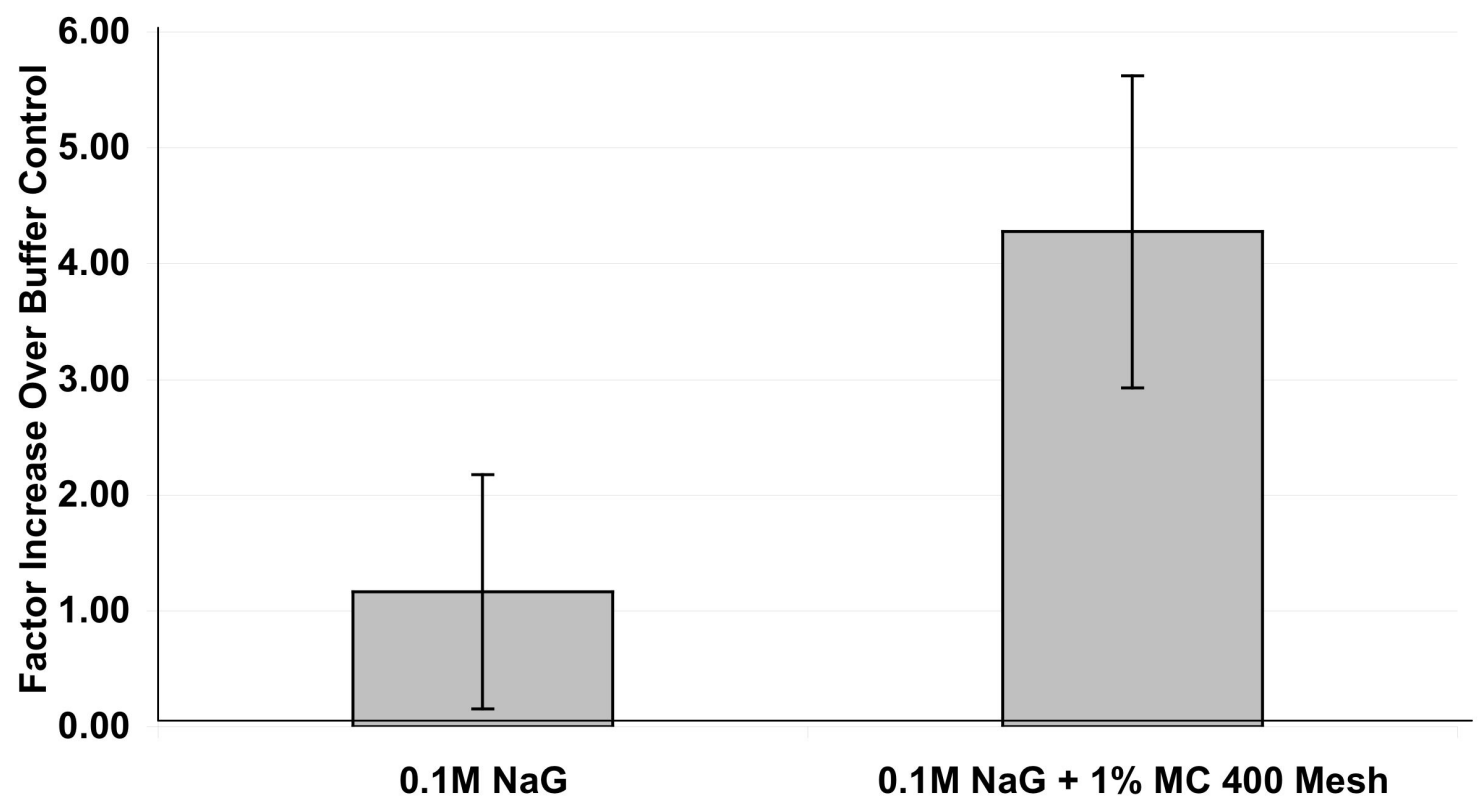

Figure 3.6: Effect of increasing assay viscosity.

In this assay the chemotactic response of $1 \times 10^{7}$ cells / $\mathrm{ml}$ to $0.01 \mathrm{M} \mathrm{N}$-acetylglucosamine $(\mathrm{NaG})$ was measured in the presence and absence of $1 \% 400$ mesh methylcellulose. This solution had a viscosity of $224 \mathrm{Cpi}$ at $34^{\circ} \mathrm{C}$. The marked improvement in the chemotactic response suggests the methylcellulose facilitated chemotaxis.

e) We found the type of preparation of bovine serum albumin (BSA) in the motility buffer influenced chemotactic response. Maintaining motility throughout the assay is vital to measuring the chemotactic response. We found motility was better preserved in buffer made with re-crystallized bovine serum albumin (BSA) (Fraction IV), than the BSA (Fraction V) that is used to make BSK II media. Fatty acid free BSA preparations seemed to shorten the period of time cells remained mobile. Because crystallized BSA greatly improved the chemotactic response, it is used to make motility buffer (Figure 3.7) (53). 
Chemotaxis to NaG by B31 and CheA2-, in Motility Buffers of Different BSA

Preparations

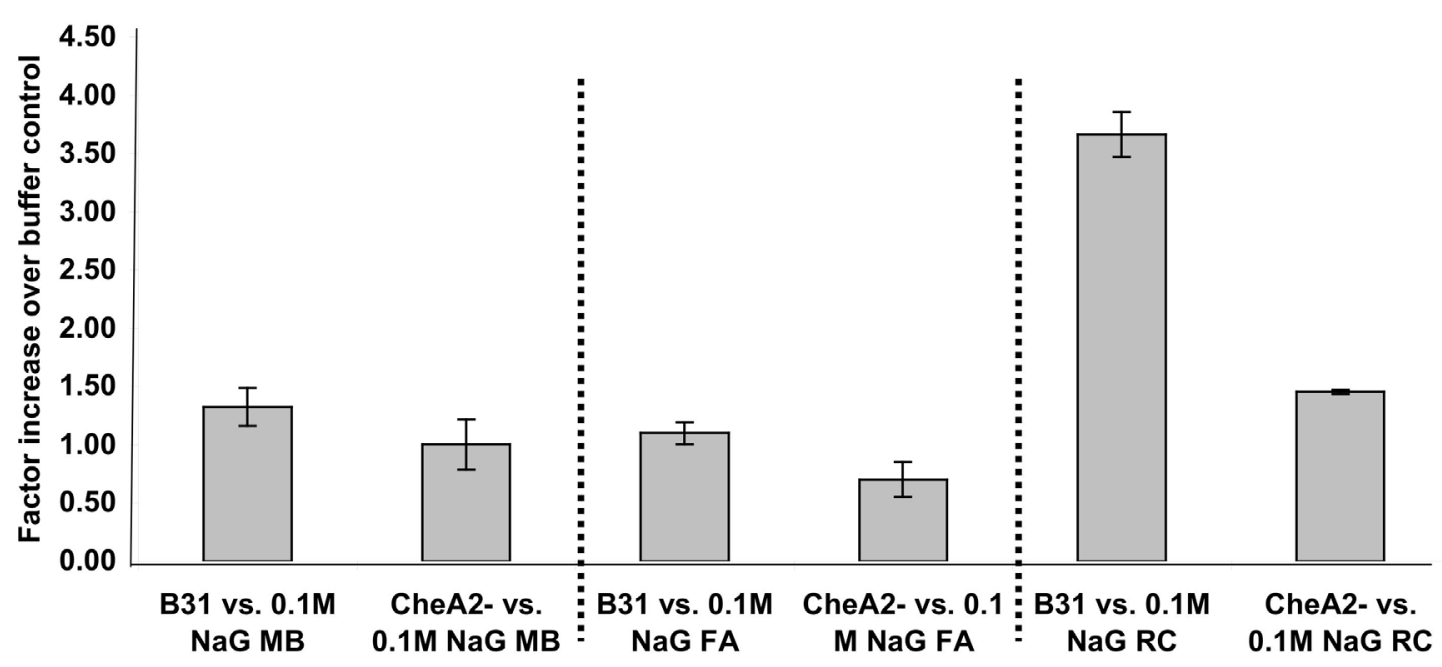

Figure 3.7: The chemotactic response of cells in preparations of motility buffer made with different BSA formulations.

$M B=$ fraction $V B S A, F A=$ Fatty acid free , and $R C=$ Fraction IV (recrystallized) BSA. CheA2- indicates the response to the non-chemotactic cheA2 mutant.

Recrystallized BSA increased the chemotactic response of cells in the assay.

f) To determine when in the growth curve B. burgdorferi would demonstrate the most rigorous chemotactic response, daily chemotaxis assays were performed from a single large culture (Figure 3.8). Data indicated that cells in late log phase were the most chemotactic when they were dividing every 9.33 hours. Cells grown from a stationary phase incoulum of $2 \mu \mathrm{l} / \mathrm{ml}$ in fresh media took 72 hours to reach this density. 
B. burgdorferi Chemotactic response to $0.01 \mathrm{M} \mathrm{NaG}$ and $0.1 \mathrm{M} \mathrm{NaG}$

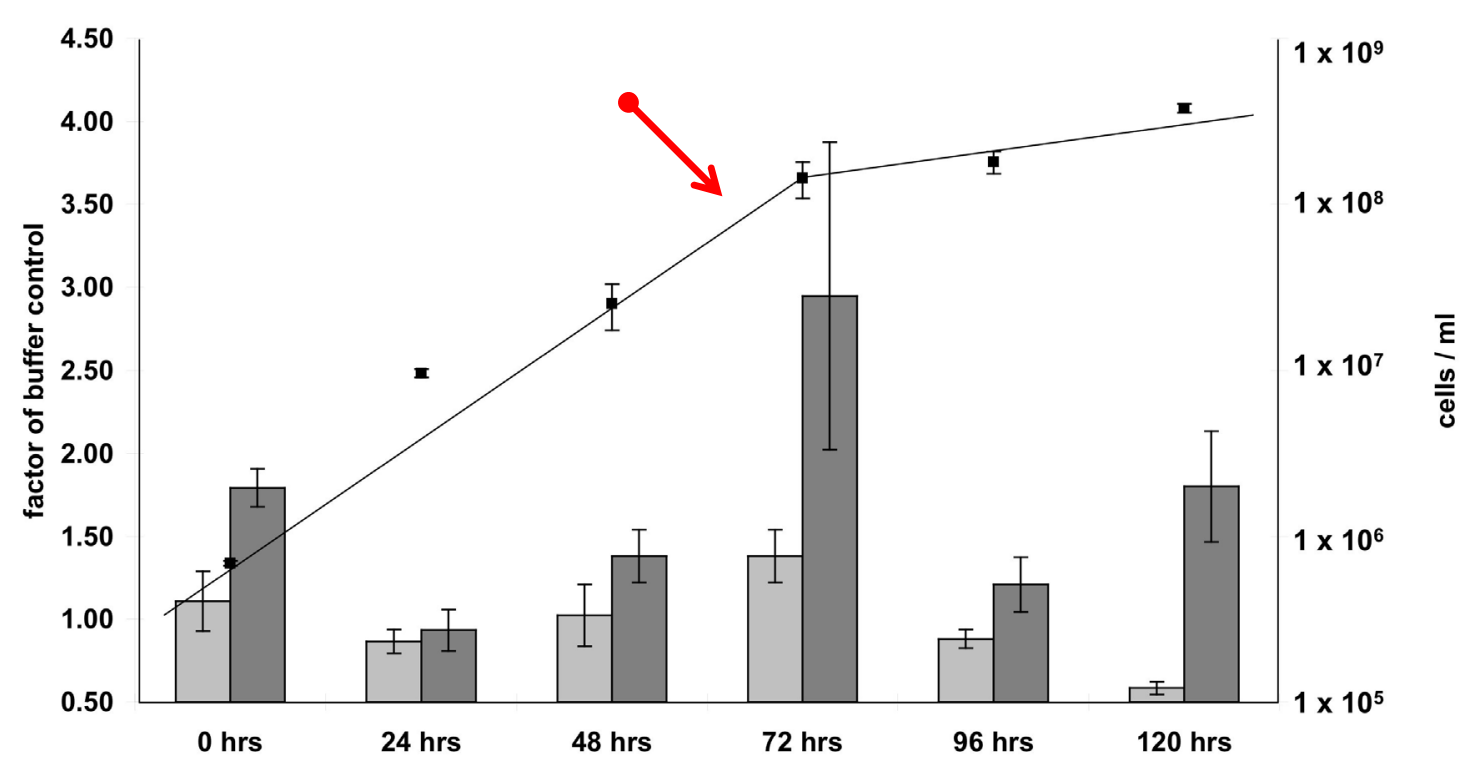

Figure 3.8: The chemotactic response as a function of culture age. Every 24 hours the chemotactic response was measured to 0.01 and $0.1 \mathrm{M}$ $\mathrm{N}$-acetyl-glucosamine $(\mathrm{NaG})$. The chemotactic response is illustrated on the left hand $\mathrm{Y}$ axis. Light grey and dark grey bars correlate to the chemotactic response to 0.01 and $0.1 \mathrm{M} \mathrm{N}$-acetyl-glucosamine (NaG), respectively. The thin black line is the growth curve, plotted on the right hand $Y$ axis. These results indicate that cells in late log phase (arrow) are the most chemotactic in this assay. The average division time during log phase was 9.33 hours.

g) The final optimization was "starving" the cells in motility buffer before running the assay (Figure 3.9). The hypothesis being, if the cells were in acute need of nutrients, they perhaps would be more chemotactic. We noted only a decline in the sensitivity of the assay by starving the cells up to 150 minutes before starting the assay. These experiments optimized the capillary tube assay to $B$. burgdorferi, and will facilitate measurement of chemotaxis in other fastidious slow growing bacterial species. 
Chemotactic Response of Prestarved Cells

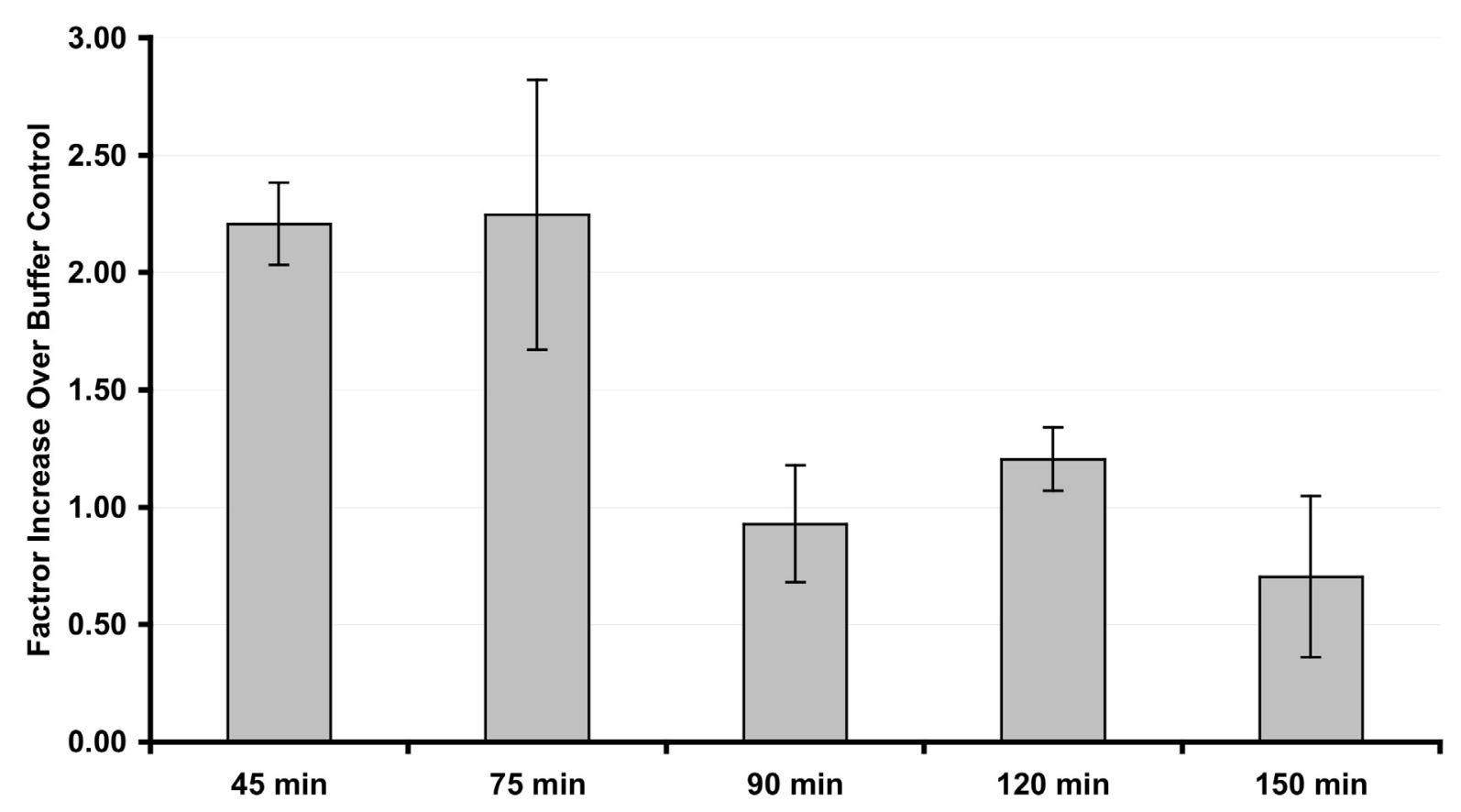

Figure 3.9: The chemotactic response of cells starved in motility buffer before beginning the assay.

The chemotactic response to $0.1 \mathrm{M} \mathrm{N}$-acetyl-glucosamine was measured in cells that were starved for $45,75,90,120$, or 150 minutes. The chemotactic ability of $B$. burgdorferi declines with starvation.

\section{The B. burgdorferi mutant LC-A2 served as a non-chemotactic control. A}

mutation in che $A$ interrupts the chemotaxis signal transduction cascade (32).

The cheA2::kan mutant of $B$. burgdorferi is non-chemotactic as measured by swarm plate and capillary tube assay (144). Recently, LC-A2 was

complemented in trans (Bakker, Li, Miller, Cunningham, and Charon, in preparation). To determine if cheA2 complementation restores chemotaxis, two different assays were used. The swarm plate assay indicated that wild type and the cheA2 complemented strain formed 17 to $18 \mathrm{~mm}$ diameter swarms, compared to the 3-5 mm diameter swarms for the LC-A2 strain. The capillary tube assays gave congruent results as shown in Figure 3.10. 
Defined attractants for $\boldsymbol{B}$. burgdorferi. We tested several compounds for chemoattractant activity. Compounds were chosen based on known nutritional requirements and/or the presence of a putative transporter in the genome. $\mathrm{N}$ acetyl-glucosamine, glucosamine, and chitosan dimers (glucosamine dimers) were found to be attractants. Neither glucose (234) nor N-n-diacetyl-chitobiose acted as an attractants although they are transported into the cell $(45,76,273)$. These are the first defined attractants for $B$. burgdorferi and they were used to measure the chemotactic response of the wild type and LC-A2. The cheA2complemented strain was tested with $\mathrm{N}$-acetyl-glucosamine. Data indicate that LC-A2 was non-chemotactic to all tested compounds. Complementation of LCA2 with the wild type gene restored chemotactic activity to $\mathrm{N}$-acetyl-glucosamine showing restoration of the phenotype (Figure 3.10) (Table 3.2).

\begin{tabular}{lll} 
Attractant / Concentration & Average Response & $\mathrm{N}$ \\
\hline N-acetyl-glucosamine 0.01 M & $2.27 \pm 1.31$ & 48 \\
N-acetyl-glucosamine 0.1 M & $3.16 \pm 1.08$ & 156 \\
Chitosan dimers 0.01M & $3.15 \pm 1.42$ & 76 \\
Chitosan dimers 0.1M & $4.96 \pm 2.22$ & 10 \\
N-n-diacetyl-chitobiose 0.01M & $1.13 \pm 0.55$ & 64 \\
Glucosamine 0.1M & $4.23 \pm 1.69$ & 93 \\
Glucose 0.1M & $1.78 \pm 0.79$ & 18 \\
\hline
\end{tabular}

\section{Table 3.2: Average response of B. burgdorferi strain B31A to different chemoattractants}

and concentrations over a period of 24 months. Average Response is listed as factor increase over buffer control \pm one standard deviation. $\mathrm{N}$ is the total number of experiments performed with each attractant.

Several other compounds have been tested with negative or mixed results (Table 3.3). The amino acids serine and glycine are notable examples. Additionally, a dilute solution of ethanol $(0.5 \%)$ acted as an attractant. Not only was ethanol an attractant by itself, it also acted synergistically with other attractants, notably chitosan dimers and rabbit serum. The tri-peptide His-Pro-Leu was tested for 
attractant activity due the presence of an oligopeptide transporter in the genome and reports of attractant activity to these compounds in other bacterial species $(76,151,161)$. However, His-Pro-Leu did not act as an attractant at tested conditions. While intriguing, results with ethanol were not followed up due to complexities that arise when looking at attractant mixtures.

\begin{tabular}{llll}
\hline Attractant & Relative & Attractant & Relative \\
& Response & N-acetyl-glucosamine & ++ \\
\hline Serine & - & $0.5 \%$ Rabbit Serum & + \\
Glycine & - & Chitosan Dimers & ++++ \\
$\beta$-Alanine & - & Histidine-Proline- & - \\
Ethanol 0.5\% & ++ & Leucine & \\
& & N-n-diacetyl-chitobiose & - \\
Glutamate & ++ & Glucose & - \\
Putricine & + & Glucosamine & +++ \\
Spermidine & + & &
\end{tabular}

Table 3.3: Relative Response to screened chemoattractants in the capillary tube assay.

+'s indicate a stronger positive chemotactic response of wild type B31 cells, whereas -'s indicate no significant response. A positive chemotactic response was considered an average response greater than 2 times the buffer control. Unless otherwise noted all attractants were tested at $0.1 \mathrm{M}$. (M. Miller and R. Bakker unpublished) 
N-Acetyl-glucosamine

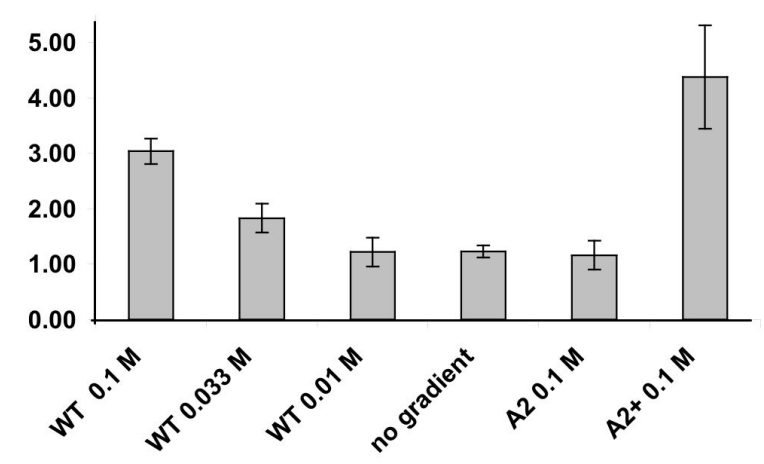

Chitosan Dimers

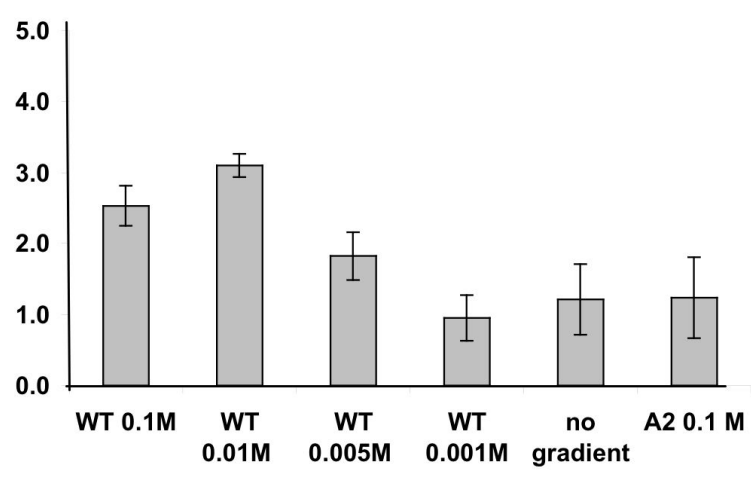

Glucosamine

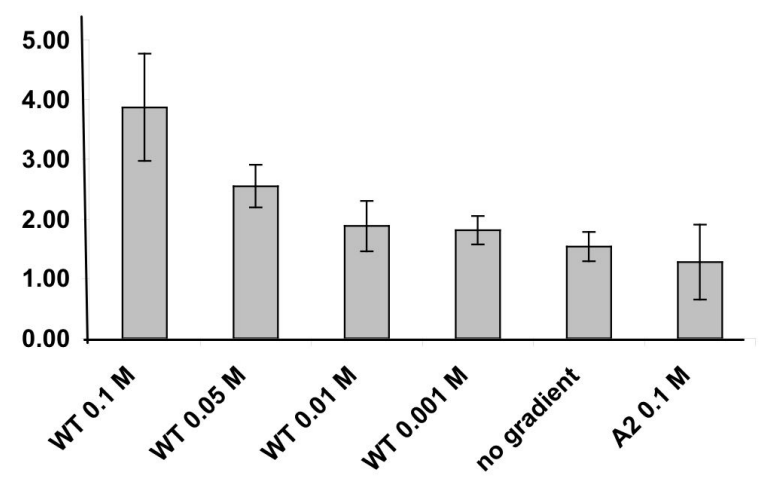

N-n-Diacetyl Chitobiose

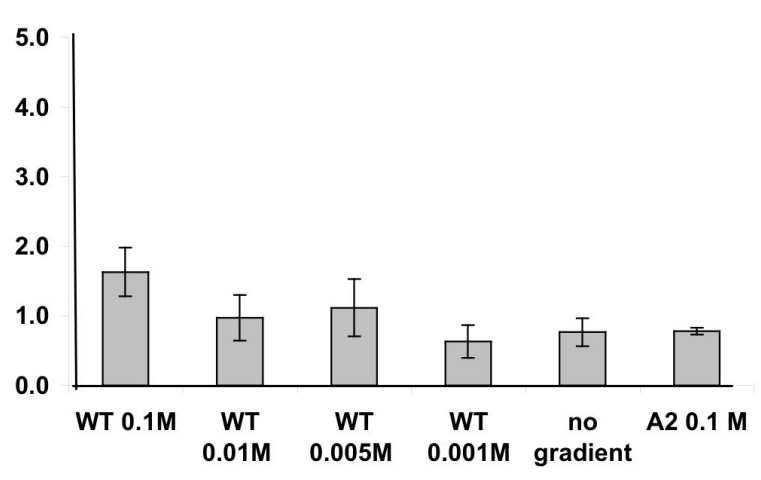

Glucose

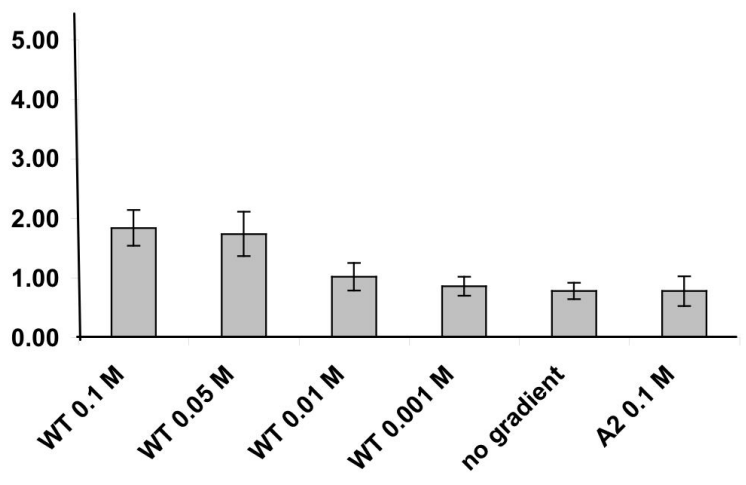

Figure 3. 10: The Response Profile of Wild-type (B31), LC-A2 and cheA2 complemented

strains to N-Acetyl-glucosamine, chitosan dimers, N-n-diacetyl-Chitobiose, glucose and glucosamine. For each of these compounds, a dose response, a no gradient control, and a non-chemotactic control were done. Cell strain and concentration, or no gradient of attractant is noted on the $\mathrm{X}$-axis while the $\mathrm{Y}$-axis is expressed in terms of factor increase over buffer control. The no gradient control was performed with the highest concentration of chemoattractant tested. $\mathrm{WT}=$ wild type, $\mathrm{A} 2$ = cheA2::kan, $\mathrm{A} 2+=$ cheA2::kan in trans complemented. 
Translational velocity. We were concerned that the tactic response measured was unrelated to chemotaxis. While results with LC-A2 (cheA2 mutants) and no gradient controls suggest this is unlikely, the measured response could be due to a simple increase in swimming speed in the presence of a nutrient. To test for this possibility, cells in motility buffer, $0.1 \mathrm{M} \mathrm{N}$-acetyl-glucosamine, and $0.1 \mathrm{M}$ glucosamine were tracked at time zero and 120 minutes later. There was no statistically significant difference in the swimming speeds of these groups. All were in the range of 2 to $4 \mu \mathrm{M}$ per second range ( $N=8$ to 10 cells), consistent with previously reported results (Table 3.4 )

\begin{tabular}{lll} 
& 0 minutes & 120 minutes \\
\hline Buffer & $2.39 \pm 1.03 \mu \mathrm{m} / \mathrm{sec}$ & $2.45 \pm 1.14 \mu \mathrm{m} / \mathrm{sec}$ \\
$0.1 \mathrm{M} N$-acetyl-glucosamine & $2.88 \pm 0.86 \mu \mathrm{m} / \mathrm{sec}$ & $1.51 \pm 0.37 \mu \mathrm{m} / \mathrm{sec}$ \\
$0.1 \mathrm{M}$ Glucosamine & $2.67 \pm 0.88 \mu \mathrm{m} / \mathrm{sec}$ & $3.36 \pm 2.05 \mu \mathrm{m} / \mathrm{sec}$ \\
\hline
\end{tabular}

\section{Table 3.4: Translational velocities one standard deviation} of B. burgdorferi B31 in a 224Cpi solution of methylcellulose and motility buffer with and without the attractants $\mathrm{N}$-acetyl-glucosamine and glucosamine at times 0 and 120 minutes. $\mathrm{N}=8$ to 10 cells for each average.

\section{Discussion}

These studies define the capillary tube chemotaxis assay for B. burgdorferi and introduce the Volocity tracking system to prokaryotic motion analysis. In addition, we identified the first defined attractants for $B$. burgdorferi. Using the capillary tube as well as cell tracking assays we show that $B$. burgdorferi's ability to reverse is related to chemotaxis, as the cheA2 mutant is non-chemotactic and unable to reverse. In this discussion I will present the significance of known chemoattractants, justify how we arrived at the compounds and concentrations used, and outline future work that is now possible in light of these findings.

Previously, chemotaxis in $B$. burgdorferi was commonly assayed with swarm plate assays. The more rapid capillary tube assay was labor intensive due to enumeration by Petroff-Hausser counting, thus limiting the number of assays that 
could be performed. Enumerating spirochetes from these assays by flow cytometry offered many advantages. By using flow cytometric enumeration, five to ten times as many assays can be easily completed in a day. These assays are less labor intensive and less subject to experimental error than capillary tube assays enumerated by Petroff-Hausser counting. We expect that the optimization of this assay will be helpful in enumerating not only $B$. burgdorferi but also other spirochete and slow growing bacterial species.

This work found the first defined attractants for B. burgdorferi. These include Nacetyl-glucosamine, glucosamine, and chitosan dimers. N-acetyl-glucosamine is an essential nutrient for Borrelia species, metabolized for energy, and mobilized for cell wall synthesis $(18,76,180,273)$. Hyaluronates found in mammalian connective tissue are $50 \% \mathrm{~N}$-acetyl-glucosamine. This suggests that $\mathrm{N}$-acetylglucosamine could be available as breakdown product in joint tissues (273 and references within, 287). These observations are perhaps of clinical significance, as Lyme disease was first described as a rheumatoid arthritis like disease (253, $255,256)$. It was these characteristics that led to our hypothesis that $\mathrm{N}$-acetylglucosamine would act as a chemoattractant.

Defining significant chemotaxis. The chemotactic response measured by the capillary tube assay is read relative to the buffer control. The number of cells entering buffer filled tubes is compared to the number of cells entering tubes containing putative chemoattractants. Therefore, a significant response was expressed as a ratio of the number of cells in the attractant filled tubes as compared to the buffer filled tubes. Adler et. al. defined the "threshold" of a positive chemotactic response to be two standard deviations above the buffer controls in experimental tubes (172). Other workers used a simple factor of twice the buffer control to determine a significant chemotactic response $(124,126$, 177). The higher threshold of twice the number of cells in the attractant filled tubes as the buffer filled tubes was chosen as the level of significance. E. coli or Pseudomonas aeruginosa show responses of 10 to 90 times increase over buffer 
control $(1,124,171,172)$. Spirochetes, as compared to other bacteria, have not shown as strong a chemotactic response. Spirochaeta aurantia show 6 to 10 fold increases over buffer control, while Brachyspira hyodysenteriae show responses of 2 to 16 times $(95,126)$. Data indicate that $B$. burgdorferi has responses in the range of 2 to 5 times the buffer control. Due to this lower overall gain, the more rigorous standard was chosen to ensure a true chemotactic response was measured (Table 3.2).

\section{Because relatively high concentrations of attractants were used in the capillary tube assay, we were concerned that the attractant gradient} dissipated quickly. Additionally, our assay used a $70 \mu$ capillary tube with an aperture 7.6 times the area of a $1 \mu$ l capillary tube making early dissipation of the gradient a concern (1). A dissipated attractant gradient would lead to no chemotaxis occurring as was seen in no gradient controls. Concentration at several points were estimated using (182):

$$
c=(C)(a / r) \operatorname{erfc}\{(r-a) /[2 \sqrt{ }(D t)]\}
$$

where $\mathbf{c}$ is concentration at any time $\mathbf{t}$ and distance $\mathbf{a}$ away from mouth of tube. Time $\mathbf{t}$ is measured in seconds after diffusion begins, $\mathbf{a}$ is radius of the capillary tube aperture, $\mathbf{r}$ is distance to the aperture, $\mathbf{C}$ is concentration of attractant in the capillary tube, $\mathbf{D}$ is the diffusion constant $1 \times 10^{-3} \mathrm{~mm}^{2} / \mathrm{s}$, and erfc is the error function as calculated by MSExcel.

$$
\operatorname{ERFC}(x)=2 / \sqrt{ } \text { pi } \int e^{-t^{\wedge} 2} d t=1-\operatorname{ERFC}(x) \quad \int \text { from } x \text { to } \infty
$$

Given the microfuge tubes are $9 \mathrm{~mm}$ in diameter and the $70 \mu \mathrm{l}$ capillary tube has an interior diameter of $1.1 \mathrm{~mm}$, a maximal diffusion distance of $4.5 \mathrm{~mm}$ and an a value of $0.055 \mathrm{~mm}$ were used. The diffusion coefficient was estimated from the rate of diffusion of a small molecule in water. Using this equation, assuming that the concentration in the capillary tube remains the same, and the diffusion 
coefficient applies at $33^{\circ} \mathrm{C}$, concentration curves were calculated at 6 time points as shown in Figure 3.11.

Attractant Concentration at a Point in Time vs. Distance from the Mouth of a 70ul Capillary Tube in a $2 \mathrm{ml}$ microfuge tube

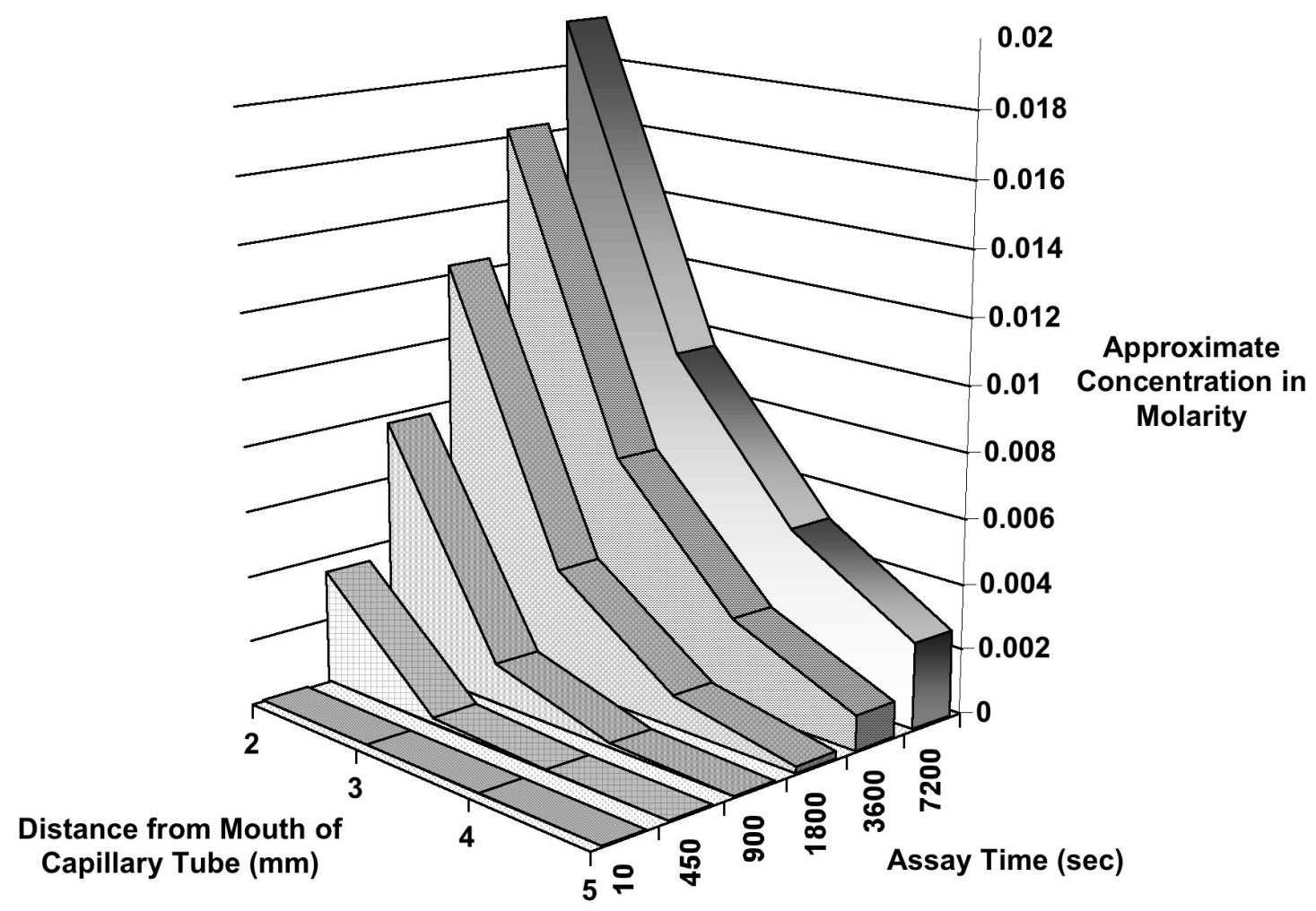

Figure 3.11: Estimation of the Attractant Gradient.

Estimated concentration of the attractant ( $y$ axis) at a distance ( $x$ axis) and time ( $z$ axis) in the chemotaxis assay. Concentration is moles, distance is in $\mathrm{mm}$ and time is in seconds. At 2 hours (7200 seconds) a gradient of attractant is maintained.

These calculations indicate that at 2 hours an attractant gradient is maintained in the capillary tube.

\section{High concentrations of chemoattractants are often necessary to elicit a} measurable response. Concentrations of $0.1 \mathrm{M}$ are not inconsistent with what is required for an attractant response in other bacterial species. Maximal responses to alanine, asparagine, glycine, and methionine are not reached until 
concentrations of $0.1 \mathrm{M}$ are used in $E$. coli (171). Spirochaeta aurantia and Brachyspira hyodysenteriae require $0.1 \mathrm{M}$ for a maximal response to 12 of 21 or 3 of 9 defined chemoattractants tested, respectively $(95,126)$. These examples demonstrate that use of high concentrations of chemoattractants would not be unexpected for $B$. burgdorferi.

The significance of having defined attractants for $B$. burgdorferi lies in what experiments are now possible. Having defined attractants facilitates the search for other attractants. Additionally, it allows for precise characterization of the chemotactic response to individual cells to a specific attractant. This is important for characterizing how the cells coordinate flagellar motors to translate in the direction of an attractant. Micro diffusion of attractants near one cell pole using iontophoresis will allow characterization of how the distant cell pole responds to the presence of an attractant at the other cell pole $(47,228)$. Data generated hold the intriguing possibility that $B$. burgdorferi could sense attractants spatially as well as, or instead of, temporally as discussed in the Introduction $(65,269)$. The experiments, which are now possible with defined attractants, in combination with mutants generated in chemotaxis genes, will greatly advance the understanding of how $B$. burgdorferi sense and respond to their environment.

In sum, the chemotactic ability of three B. burgdorferi strains, the wild-type (B31), LC-A2, and the complemented cheA2 mutants were studied. Because the complemented strains behaved like the wild-type in chemotaxis assays while the cheA2 mutant is non-chemotactic, the mutant phenotype in LC-A2 (cheA2) is due to che $A 2$ inactivation and not polar effects on gene expression. Furthermore, the responses to the attractants $\mathrm{N}$-acetyl-glucosamine, glucosamine, and chitosan dimers were CheA2 dependant, showed a dose response, and required an attractant gradient indicating chemotaxis was measured. Through these studies, we showed that spirochete enumeration by flow cytometry is a viable alternative to Petroff Hausser counting, and describe the first defined attractants for $B$. burgdorferi, the causative agent of Lyme disease. 
Acknowledgements:

WVU School of Medicine MD/PhD program to RB

PHS Grant \# Al29743 to NC

The WVU Flow Cytometric Core Facility is Supported by PHS Grant \# RR16440 


\section{Discussion}

In this section the significance of my results to the $B$. burgdorferi literature will be explored. The discussion will follow the chapter chronology. These sections will focus on techniques developed, the significance of the data generated, and possible future experiments. The discussion begins with characterization of the cheA mutants with the Hobson BacTracker, and concludes with chemotaxis assays enumerated with flow cytometry and validated using the Volocity tracking software package.

\section{Asymmetrical flagellar rotation in Borrelia burgdorferi cheA mutants.}

My major contribution to the work in Chapter 2 was developing the motion analysis procedure using the Hobson BacTracker. I then used this system to characterize the cheA2 mutant. The capabilities and use of this hardware / software chimera are outlined in the Introduction. Automated functions of this package, designed for $E$. coli, did not work for $B$. burgdorferi due to the cells elongated morphology and slow speed. Therefore, a secondary software package from Hobson tracking systems known as $X Y$ tracker was utilized for tracking cells. This package gave the $X Y$ coordinates of the centroid every $1 / 60^{\text {th }}$ of a second. The centroid is the geographic center of the cell found by integrating over the surface. The surface was defined as a group of adjacent pixels of similar brightness intensity. The intensity threshold can be adjusted so a given cell is recognized as a single object and not a series of objects. This paradigm is the basis for tracking with the Hobson BacTracker as well as the new software package Volocity. Difficulty arises in using the BacTracker given the fixed field size, cell brightness requirements, antiquated data input/output capabilities, and data sorting requirements. Every object in the field is tracked generating a great deal of superfluous data. To determine what data are significant, one must graph all tracks to see if the pattern matches the motion of the cell. Once the track is found distances and velocities are calculated using the 
Pythagorean theorem $\left(a^{2}+b^{2}=c^{2}\right.$, where $\mathbf{a}$ and $\mathbf{b}$ represent the length of sides of a triangle and $\mathbf{c}$ represents the length of its hypotenuse). These limitations require that the cell be the only object moving in a darkfield image, thus limiting the population of cells suitable for tracking. Finally the system requires that all image sequences be shot at 60 interlaced $320 \times 760$ pixel frames per second precluding digital image capture or analysis of compressed sequences. These limitations make tracking with the Hobson system labor intensive. They also introduce selection bias into what cells can be tracked. These difficulties led to further interest in more adaptable tracking systems.

The significance of our findings in Chapter 2 is that asymmetrical flagellar rotation is not dependant upon the chemotaxis system. Specifically, the data do not support the hypothesis that a gradient of CheY-P is the basis for asymmetrical flagellar rotation. As mentioned in the Introduction and Chapter 2, in order for spirochetes to translate, the sub-polar flagellar motors must rotate in opposite directions as observed from the center of the cell. The gradient hypothesis states that this asymmetry is due to a higher concentration of phosphorylated CheY (CheY-P) at one cell pole relative to the other. Thus, the bundle of periplasmic flagella at the cell pole with the low CheY-P would rotate $\mathrm{CCW}$, and the other with the higher concentration would rotate CW $(47,89,144)$.

Our findings do not support the gradient hypothesis. Cells with a cheA2 (LC-A2), and both cheA1 and cheA2 (LC-A1A2) inactivated, constantly run. Mutants in cheA, especially LC-A1A2, likely cannot generate CheY-P. With no CheY-P generated, no internal CheY-P gradient could form. These results indicate that asymmetrical flagellar rotation is unrelated to CheY-P concentration. As discussed in Chapter 2, we proposed that asymmetry is related to differences in the motors at each end of the cell.

Examination of the two $B$. burgdorferi che $A$ genes reveals that they are likely of different phylogenetic origin. che $A 1$ has the highest similarity to cheA2 and 
cheA1 of Rhodobacter sphaeroides (35\% identity) and Vibrio cholerae (35\% identity), respectively, inferring it is a more recent acquisition from the proteobacteria. The $B$. burgdorferi che 22 gene had the highest similarity to the che $A$ genes of the spirochete genus Treponema (144). Additionally, cheA mutants in Treponema denticola exhibit a non-chemotactic phenotype (156). These bioinformatic and genetic findings parallel empiric observations. Specifically, the single cheA1 mutant was phenotypically wild type. The function of CheA1 is unknown, however, Western blot analysis indicates it is expressed (144). Several bacterial species have multiple cheA genes, notably Vibrio cholera, Rhodobacter sphaeroides, Pseudomonas aeruginosa, and Chromobacterium violaceum $(73,92,163,198,207,278)$. While many await characterization, it is known that mutants in some, not all, cheA species in $V$. cholera, $R$. sphaeroides, and $P$. aeruginosa are attenuated with respect to chemotaxis $(73,92,163,207,278)$. In contrast, LC-A1 (cheA2) and LC-A1A2 double mutants (cheA1A2) were unable to reverse and were non-chemotactic. These results suggest the well-conserved operon containing cheA2 is central to B. burgdorferi chemotaxis.

Because the che $A$ double mutants exhibited polar effects, the possibility that the phenotype of cheA2 mutants was not due solely to cheA2 inactivation was explored. Specifically, the motility apparatuses must be intact to measure the chemosensory response. To test if motility structures were intact, cells or cell lysates of wild type, LC-A2, and LC-A1A2 were examined with electron microscopy, Western blot, and phase contrast microscopy (144). First, electron microscopy indicated cheA2 mutants had periplasmic flagella at both poles. Furthermore, Western blot showed che $A$ single mutants produced the same quantity of flagellar proteins (FlaA and FlaB) as the wild type. Finally, to examine if both polar bundles of flagella were functional, phase contrast microscopy was used to examine cells adhered to the glass in the center of the cell. Examining the rotation of these cells frame-by-frame shows that both cell poles are able to rotate independently of one another. In sum, these results suggest that the 
che $A 2$ mutants had no noticeably altered phenotype with respect to motility function. Complementation of the cheA2 mutant, as illustrated in Chapter 3 , again suggested the non-chemotactic phenotype of cheA2 mutants is due to the loss of chemosensory ability and not changes in the motility organelles.

The chemotactic ability of these organisms was tested using swarm plate as well as capillary tube assays. The swarm plate assay is a soft agar plate where organisms are deposited on the agar and as the bacteria consume attractants in the immediate area they swim out to higher concentrations of attractant, provided the cells can sense and respond to the attractant. Mutants without CheA2 were non-chemotactic using this assay. It is important to note that the capillary tube assays performed in Chapter 2 utilized a different protocol. Assays in Chapter 2 were performed before data from optimization protocols in Chapter 3 were available. We do not believe this protocol change affected the final results as cheA2 mutants remained non-chemotactic throughout a large number of assays with different attractants using both protocols.

In sum, by careful analysis of wild type and cheA mutants, LC-A1, LC-A2, and LC-A1A2, data shows CheA2 is intimately involved in chemotaxis. Using the Hobson BacTracker, B. burgdorferi demonstrated an intrinsic polarity to flagellar bundle rotation. Mutants in cheA2 translated in one only direction, indicating motors at the poles rotate counter-directionally to one another. While most studied bacterial species have flagellar motors that rotate in a default CCW manner, the data indicate $B$. burgdorferi have one pole of motors rotating in a default $\mathrm{CCW}$ and the other $\mathrm{CW}$. Furthermore, this is the first direct evidence to address and negate the hypothesis that a gradient of CheY-P generated by the chemotaxis apparatus was responsible for the counter directional rotation of spirochete flagellar motors. 


\section{Identification of specific chemoattractants for Borrelia burgdorferi: A flow cytometric based chemotaxis assay.}

We developed the flow cytometric based enumeration protocol as an alternative to direct Petroff Hausser $(\mathrm{PH})$ counting. $\mathrm{PH}$ counting is labor intensive, statistically ineffective for cell populations less than $1 \times 10^{6}$ cells $/ \mathrm{ml}$, and has a very low daily throughput of 9 to 12 capillary tubes per day. Using flow cytometry for enumeration, counting of dilute solutions was performed and throughputs of 60 to 100 tubes a day were routinely achieved. The high throughput of the flow cytometric protocol allowed for optimization, as discussed in Chapter 3, of several variables of the capillary tube assay to $B$. burgdorferi. Therefore the capillary tube data presented in Chapter 2 was collected with a different protocol than in Chapter 3.

In order to track cells more effectively, we consulted with the software engineers at Improvision who tweaked the design of the Volocity software package to track B. burgdorferi. Volocity, as presented in the Introduction, has a very user-friendly interface and allows for much more rapid tracking of cells. Additionally, this interface has allowed several members of the laboratory to track swimming cells. Finally, the Volocity package provided an important and easier means to determine the translational velocity of swimming cells.

Once the capillary tube assay was optimized, we began to screen compounds hypothesized to be attractants. These compounds included known nutritional requirements and transported molecules. Early in the process, we discovered that chitosan dimers and $\mathrm{N}$-acetyl-glucosamine acted as attractants. These were the first defined attractants for $B$. burgdorferi. $\mathrm{N}$-acetyl-glucosamine, while not as effective an attractant as chitosan dimers, was used due its availability and economy. Once the optimizations were completed, screening other compounds that are known nutrients, had putative transporters encoded in the $B$. burgdorferi genome, or were similar in structure to known attractants was begun. Glucose, 
$\mathrm{N}$-n-diacetyl chiotobiose, serine, glycine, and beta-alanine were not attractants. Glucosamine, chitosan dimers, glutamate, and N-acetyl-glucosamine are stronger attractants. Putricine and spermidine have transporters encoded in the genome, but do not produce very robust chemotactic responses $(45,76)$. This screen for attractants is ongoing. Knowledge of a range of attractants is important to deduce the function of chemotaxis components, especially the methyl accepting chemotaxis proteins.

There is some suggestion that attractants could provide insight into clinical disease. The connective tissue components glycosaminoglycans are abundant, especially in joint tissue (287). Two important forms are hyaluronates are made up of D-glucuronate and $\mathrm{N}$-acetyl glucosamine with a $\beta(1,3)$ linkage and keratan sulfate made up of galactose and $\mathrm{N}$-acetyl-glucosamine-6-sulfate with a $\beta(1,4)$ linkage. $\mathrm{N}$-acetyl-glucosamine polymers with a $\beta(1,4)$ linkage make up chitin, the substance of tick exoskeletons (132). The molecular similarity of these molecules and the natural history of the infection suggest that chemotaxis toward breakdown products or intermediates of these connective tissue components could play a role in disease transmission and clinical manifestations.

To ensure that chemotaxis was being measured, we used the cheA2 mutant (LCA2), a no gradient control, individual cell tracking, and calculated the gradient concentration during the assay. Mutants in cheA2 were shown in Chapter 2 not to reverse, be non-chemotactic by capillary tube and swarm plate assay, and exhibit a constantly running phenotype similar to that of $E$. coli cheA mutants. Based on these observations, we propose that CheA2 plays a similar role in $B$. burgdorferi as does CheA in $E$. coli linking the activities of the chemosensory and motility structures. Therefore, non-chemotactic cheA2 mutants should not and did not exhibit a chemotactic response in the presence of attractants. Because chemotaxis occurs only in a concentration gradient, placing the attractant in the cell pool as well as in the capillary tube quashes any attractant gradient. In this experimental setup known as a no gradient control, no chemotaxis took place as 
expected. It was possible that $B$. burgdorferi responded to attractants by simply increasing translational velocity. To control for this scenario, cells in attractant and buffer were filmed and tracked at time points corresponding to the beginning and end of an assay. There was no significant difference in translational velocities between wild-type cells in buffer versus those in attractant solutions. These results suggest that chemotaxis was measured and that the reported results are not artifactual.

The significance of having defined attractants for $B$. burgdorferi lies in what experiments are now possible. Having defined attractants gives good positive controls for other chemotaxis assays, which facilitates the search for other attractants. Additionally, it allows for precise characterization of the translational response to attractants. This is important to characterizing how the cells coordinate these motors to translate in the direction of an attractant. Microdiffusion of attractants near one cell pole will allow characterization of how the distant cell pole responds to the presence of an attractant at the other cell pole (228). These data hold the intriguing possibility that $B$. burgdorferi could sense attractants spatially as well as, or instead of, temporally as discussed in the Introduction $(65,269)$.

The protocols developed for this work are now standard laboratory protocols. The primary use for flow cytometry in the Charon laboratory is enumeration of cells from capillary tube assays. However, enumeration is finding broader application. For example, flow cytometry is used to generate growth curves to ensure that different media preparations do not affect growth rate. Enumeration is now an important step before cell lysates are prepared for several molecular techniques. This technique insures that a similar number of cells go into a given protein or nucleic acid preparation facilitating standardization. Using a different fluorescent detector, I have screened cultures for green fluorescent protein (GFP) expression. If the gene for GFP is fused to a gene to be expressed, flow cytometry provides a fast and reliable means of screening for expression of the 
fusion protein (44). These are examples of flow cytometry applications currently used in the laboratory, but the list will likely grow with experience.

Tracking the movement of bacterial cells has proved a challenge for many laboratories, as discussed in the Introduction. By facilitating the tracking of $B$. burgdorferi, the Volocity software package designed to track motion of and within eukaryotic cells, expands its capabilities to prokaryotes. Data from the Hobson BacTracker, Volocity, or hand counting indicate that translational velocity remains in the range of 3 to $6 \mu \mathrm{m}$ per second at 33 to $37^{\circ} \mathrm{C}$ in macroscopically viscous solutions of approximately $220 \mathrm{Cpi}$. There are reports of $B$. burgdorferi translating faster, however these reports used different temperatures and viscosities $(91,131)$. Additionally, because temperature, viscosity, type of viscosity, and cell selection dramatically affect the translational speed of cells, one should be cautious in comparing data from different sources. In sum, analysis of individual cell motion provides an important control for responses measured in a population.

\section{Future work}

The understanding of chemotaxis and motility will be advanced on four fronts. First, protocols and procedures in my work in part lay the groundwork for future experiments defining the role of chemotaxis and motility in $B$. burgdorferi virulence and how this translational activity occurs. Screening for additional attractants, micro-diffusion techniques and photo-released attractant compounds will better describe the dynamics of attractant sensing $(112,113,129,228,263)$. For example, micro-diffusion techniques will allow for examination of the response to an attractant at one cell pole. This will lend insight into coordination between the poles and therefore the swimming mechanism. Photo-released attractant compounds will allow for analysis of the immediate response to attractant release. These tools provide mechanisms to study translation in wild type and specific mutants. These studies promise to lend insight into the 
coordination mechanism between the cell poles. The whole of this work could suggest a model where attractants are sensed spatially and not temporally, a first in a genetically described, culturable organism. While suggestions of this possibility are in the literature, definitive data would be seminal in bacteriology.

Secondly, functional assays will examine how $B$. burgdorferi interacts with its environment. The effect of environmental cues, such as the presence of host proteins or quorum sensing molecules could influence what compounds act as attractants $(21,225,246,258,259)$. Data generated in the course of capillary tube assay optimization as well as the natural history of a $B$. burgdorferi infection leads me to suspect that chemotaxis and motility will be affected by quorum sensing. The study of transporter proteins will lend insight into how $B$. burgdorferi bring nutrients into the cell. Perhaps, as with the phosphotransferase systems in E. coli, the transport of compounds will be integrated into to the chemotactic response $(152,155)$. The invasive behavior of strains with different genomic backgrounds will likely be measured with in vitro tissue penetration assays, as well as animal studies $(56,57,83,153,156,200)$. These studies will give insight into what signals $B$. burgdorferi senses from its environment.

Finally, further genetic characterization and proteome analysis of B. burgdorferi will provide insight into the pathogenesis of this organism as it relates to chemotaxis and motility. Traditional mutation and complementation will continue to provide data on the function of specific genes. Interesting mutant phenotypes will be analyzed with respect to protein expression, micro arrays, and reverse transcriptase polymerase chain reaction (RT-PCR) techniques. Finally, gene expression studies comparing the proteome of cells grown in different conditions can be explored. The recent development of a transposon mutational system will provide a broad range of mutants quickly and in pathogenic strains (Stewart, $P$ et. al. submitted). These mutants will greatly facilitate the study of pathogenesis as related to chemotaxis and motility in animal models. 


\section{Conclusion.}

In conclusion, the significance of my work includes:

a. Developing tools that will aid in further understanding chemotaxis and motility of $B$. burgdorferi specifically and spirochetes generally.

b. Identifying the first specific chemoattractants of $B$. burgdorferi.

C. In part helping to identify that CheA2 is involved in the chemotaxis of $B$. burgdorferi.

d. In part, finding that asymmetrical rotation of the bundles of periplasmic flagella in $B$. burgdorferi is not due to a gradient of CheY-P, and cheA2 mutants continuously ran in one direction. 
$\underline{\text { Rights and Clearances }}$ 
University of Minnesota

Twin Cities Campus Genetics, Cell Biology, and Development
Combined Department - Medical School
and College of Biological Sciences
6-160 Jackson Hall

321 Church Street S.E.

Minneapolis, MN 55455

612-624-3110

Fax: 612-626-6140

http://www.gcd.med.umn.edu

February 27, 2004

Mr. Richard Bakker

Department of Microbiology \& Immunology

Byrd Health Sciences Center

West Virginia University

PO Box 9177

Morgantown, WV 26506-9177

Dear Richard,

You have my permission to use Figure 8 of Goldstein \& Charon (1990) PNAS 87, 4895-4899, in

your doctoral dissertation. You may use it in both the hardcopy and on-line versions.

I wish you continued success in your research.

Sincerely,

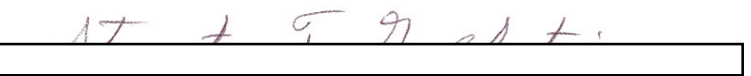

Stuart F. Goldstein

Professor 


\author{
MEdiCAL SCHOOL \\ John L. Spudich, PHD \\ Robert A. Welch Distinguished Chair in Chemistry \\ Professor and Director, Center for Membrane Biology \\ Department of Biochemistry and Molecular Biology \\ Department of Microbiology and Molecular Genetics
}

The University of Texas

Health Science Center at Houston

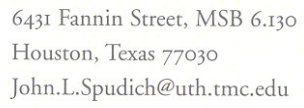

6431 Fannin Street, MSB 6.130

Houston, Texas 77030

John.L.Spudich@uth.tmc.edu

http://www.uth.tmc.edu/cmb/

February 17, 2004

Mr. Richard G. Bakker

West Virginia University

Robert C. Byrd Health Sciences Center

Department of Microbiology, Immunology and Cell Biology

2095 Health Sciences North

PO Box 9177

Morgantown, WV 26506-9177

Dear Mr. Bakker:

I give my permission for you to use figures from my paper "Quantitation of the Sensory Response in Bacterial Chemotaxis", Proceedings of the National Academy of Sciences 1975, $72: 2710-173$, both in hard copies and electronic versions of your dissertation.

Best wishes,

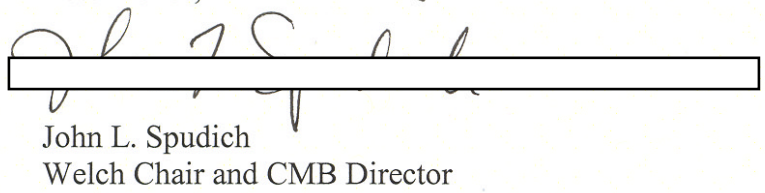

JLS/jsp 


\section{Works Cited}

1. Adler, J. 1973. A method for measuring chemotaxis and use of the method to determine optimum conditions for chemotaxis by Escherichia coli. J Gen Microbiol 74:77-91.

2. Adler, J., and M. M. Dahl. 1967. A method for measuring the motility of bacteria and for comparing random and non-random motility. J Gen Microbiol 46:161-73.

3. Adler, J., and W. Epstein. 1974. Phosphotransferase-system enzymes as chemoreceptors for certain sugars in Escherichia coli chemotaxis. Proc Natl Acad Sci U S A 71:2895-9.

4. Adler, J., and B. Templeton. 1967. The effect of environmental conditions on the motility of Escherichia coli. J Gen Microbiol 46:175-84.

5. Aldridge, P., and K. T. Hughes. 2001. How and when are substrates selected for type III secretion? Trends Microbiol 9:209-14.

6. Aldridge, P., and K. T. Hughes. 2002. Regulation of flagellar assembly. Curr Opin Microbiol 5:160-5.

7. Ames, P., and J. S. Parkinson. 1988. Transmembrane signaling by bacterial chemoreceptors: $E$. coli transducers with locked signal output. Cell 55:817-26.

8. Ames, P., C. A. Studdert, R. H. Reiser, and J. S. Parkinson. 2002. Collaborative signaling by mixed chemoreceptor teams in Escherichia coli. Proc Natl Acad Sci U S A 99:7060-5.

9. Andermann, T. M., Y. T. Chen, and K. M. Ottemann. 2002. Two predicted chemoreceptors of Helicobacter pylori promote stomach infection. Infect Immun 70:5877-81.

10. Armitage, J. P. 1999. Bacterial tactic responses. Adv Microb Physiol 41:229-89.

11. Asakura, S., and H. Honda. 1984. Two-state model for bacterial chemoreceptor proteins. The role of multiple methylation. J Mol Biol 176:349-67. 
12. Asbrink, E., and A. Hovmark. 1985. Successful cultivation of spirochetes from skin lesions of patients with erythema chronicum migrans Afzelius and acrodermatitis chronica atrophicans. Acta Pathol Microbiol Immunol Scand [B] 93:161-3.

13. Atsumi, T., Y. Maekawa, T. Yamada, I. Kawagishi, Y. Imae, and M. Homma. 1996. Effect of viscosity on swimming by the lateral and polar flagella of Vibrio alginolyticus. J Bacteriol 178:5024-6.

14. Ausubel, F. M. 1994. Current protocols in molecular biology. John Wiley \& Sons, New York.

15. Bacon, R. M., B. J. Biggerstaff, M. E. Schriefer, R. D. Gilmore, Jr., M. T. Philipp, A. C. Steere, G. P. Wormser, A. R. Marques, and B. J. Johnson. 2003. Serodiagnosis of Lyme disease by kinetic enzyme-linked immunosorbent assay using recombinant VIsE1 or peptide antigens of Borrelia burgdorferi compared with 2-tiered testing using whole-cell lysates. J Infect Dis 187:1187-99.

16. Bainer, R., H. Park, and P. Cluzel. 2003. A high-throughput capillary assay for bacterial chemotaxis. J Microbiol Methods 55:315-9.

17. Barbour, A., Burgdorfer, S. F., Hayes, Peter, O. and Aeschlimann, A. 1983. Isolation of a cultivatible spirochete from Ixodes ricinus ticks of Switzerland. Curr. Microbiology 8:123-126.

18. Barbour, A. G. 1984. Isolation and cultivation of Lyme disease spirochetes. Yale J Biol Med 57:521-5.

19. Barbour, A. G., and S. F. Hayes. 1986. Biology of Borrelia species. Microbiol Rev 50:381-400.

20. Barbour AG, H. S. 1986. Biology of Borrelia species. Microbiol Rev 50:381-400.

21. Bassler, B. L. 2002. Small talk. Cell-to-cell communication in bacteria. Cell 109:421-4.

22. Benach, J. L., E. M. Bosler, J. P. Hanrahan, J. L. Coleman, G. S. Habicht, T. F. Bast, D. J. Cameron, J. L. Ziegler, A. G. Barbour, W. Burgdorfer, R. Edelman, and R. A. Kaslow. 1983. Spirochetes isolated from the blood of two patients with Lyme disease. N Engl J Med 308:740-2. 
23. Berg, H. C. 1983. Random Walks in Biology. Princeton University Press, Princeton, New Jersey.

24. Berg, H. C., and D. A. Brown. 1972. Chemotaxis in Escherichia coli analysed by three-dimensional tracking. Nature 239:500-4.

25. Berg, H. C., and L. Turner. 1979. Movement of microorganisms in viscous environments. Nature 278:349-51.

26. Bilwes, A. M., L. A. Alex, B. R. Crane, and M. I. Simon. 1999. Structure of CheA, a signal-transducing histidine kinase. Cell 96:131-41.

27. Bono, J. L., A. F. Elias, J. J. Kupko, 3rd, B. Stevenson, K. Tilly, and P. Rosa. 2000. Efficient targeted mutagenesis in Borrelia burgdorferi. J Bacteriol 182:2445-52.

28. Bornhorst, J. A., and J. J. Falke. 2003. Quantitative analysis of aspartate receptor signaling complex reveals that the homogeneous two-state model is inadequate: development of a heterogeneous two-state model. J Mol Biol 326:1597-614.

29. Bouma, C. L., and S. Roseman. 1996. Sugar transport by the marine chitinolytic bacterium Vibrio furnissii. Molecular cloning and analysis of the glucose and N-acetylglucosamine permeases. J Biol Chem 271:33457-67. 30. Boyd, A., K. Kendall, and M. I. Simon. 1983. Structure of the serine chemoreceptor in Escherichia coli. Nature 301:623-6.

31. Bren, A., and M. Eisenbach. 2001. Changing the direction of flagellar rotation in bacteria by modulating the ratio between the rotational states of the switch protein FliM. J Mol Biol 312:699-709.

32. Bren, A., and M. Eisenbach. 2000. How signals are heard during bacterial chemotaxis: protein-protein interactions in sensory signal propagation. J Bacteriol 182:6865-73.

33. Bromley, D. B., and N. W. Charon. 1979. Axial filament involvement in the motility of Leptospira interrogans. J Bacteriol 137:1406-12.

34. Brooks, C. S., P. S. Hefty, S. E. Jolliff, and D. R. Akins. 2003. Global analysis of Borrelia burgdorferi genes regulated by mammalian host-specific signals. Infect Immun 71:3371-83. 
35. Brown, D. A., and H. C. Berg. 1974. Temporal stimulation of chemotaxis in Escherichia coli. Proc Natl Acad Sci U S A 71:1388-92.

36. Brown, R. N., and R. S. Lane. 1992. Lyme disease in California: a novel enzootic transmission cycle of Borrelia burgdorferi. Science 256:1439-42.

37. Bugrysheva, J., E. Y. Dobrikova, H. P. Godfrey, M. L. Sartakova, and F. C. Cabello. 2002. Modulation of Borrelia burgdorferi stringent response and gene expression during extracellular growth with tick cells. Infect Immun 70:3061-7. 38. Burgdorfer, W. 1984. Discovery of the Lyme disease spirochete and its relation to tick vectors. Yale J Biol Med 57:515-20.

39. Caimano, M. J., X. Yang, T. G. Popova, M. L. Clawson, D. R. Akins, M. V. Norgard, and J. D. Radolf. 2000. Molecular and evolutionary characterization of the cp32/18 family of supercoiled plasmids in Borrelia burgdorferi 297. Infect Immun 68:1574-86.

40. Canale-Parola, E. 1978. Motility and chemotaxis of spirochetes. Annu Rev Microbiol 32:69-99.

41. Canica, M. M., F. Nato, L. du Merle, J. C. Mazie, G. Baranton, and D. Postic. 1993. Monoclonal antibodies for identification of Borrelia afzelii sp. nov. associated with late cutaneous manifestations of Lyme borreliosis. Scand J Infect Dis 25:441-8.

42. Cantinieaux, B., P. Courtoy, and P. Fondu. 1993. Accurate flow cytometric measurement of bacteria concentrations. Pathobiology 61:95-7.

43. Cantwell, B. J., R. R. Draheim, R. B. Weart, C. Nguyen, R. C. Stewart, and M. D. Manson. 2003. CheZ phosphatase localizes to chemoreceptor patches via CheA-short. J Bacteriol 185:2354-61.

44. Carroll, J. A., P. E. Stewart, P. Rosa, A. F. Elias, and C. F. Garon. 2003. An enhanced GFP reporter system to monitor gene expression in Borrelia burgdorferi. Microbiology 149:1819-28.

45. Casjens, S., N. Palmer, R. van Vugt, W. M. Huang, B. Stevenson, P. Rosa, R. Lathigra, G. Sutton, J. Peterson, R. J. Dodson, D. Haft, E. Hickey, M. Gwinn, O. White, and C. M. Fraser. 2000. A bacterial genome in flux: the twelve 
linear and nine circular extrachromosomal DNAs in an infectious isolate of the Lyme disease spirochete Borrelia burgdorferi. Mol Microbiol 35:490-516. 46. Charon, N. W., G. R. Daughtry, R. S. McCuskey, and G. N. Franz. 1984. Microcinematographic analysis of tethered Leptospira illini. J Bacteriol 160:106773.

47. Charon, N. W., and S. F. Goldstein. 2002. Genetics of motility and chemotaxis of a facinating group of bacteria: The Spirochetes. Annu Rev Genet 36:47-73.

48. Charon, N. W., S. F. Goldstein, S. M. Block, K. Curci, J. D. Ruby, J. A. Kreiling, and R. J. Limberger. 1992. Morphology and dynamics of protruding spirochete periplasmic flagella. J Bacteriol 174:832-40.

49. Charon, N. W., S. F. Goldstein, K. Curci, and R. J. Limberger. 1991. The bent-end morphology of Treponema phagedenis is associated with short, lefthanded, periplasmic flagella. J Bacteriol 173:4820-6.

50. Charon, N. W., E. P. Greenberg, M. B. Koopman, and R. J. Limberger. 1992. Spirochete chemotaxis, motility, and the structure of the spirochetal periplasmic flagella. Res Microbiol 143:597-603.

51. Charon, N. W., C. W. Lawrence, and S. O'Brien. 1981. Movement of antibody-coated latex beads attached to the spirochete Leptospira interrogans. Proc Natl Acad Sci U S A 78:7166-70.

52. Chilcott, G. S., and K. T. Hughes. 2000. Coupling of flagellar gene expression to flagellar assembly in Salmonella enterica serovar typhimurium and Escherichia coli. Microbiol Mol Biol Rev 64:694-708.

53. Cohn E. J. , H. W. L., and Weare J.H. 1947. Preparation and properties of serum and plasma proteins. Crystallization of serum albumins from ethanol-water mixtures. Journal of the American Chemical Society 69a:1753-61.

54. Coleman, J. L., T. J. Sellati, J. E. Testa, R. R. Kew, M. B. Furie, and J. L. Benach. 1995. Borrelia burgdorferi binds plasminogen, resulting in enhanced penetration of endothelial monolayers. Infect Immun 63:2478-84.

55. Collares-Pereira, M., S. Couceiro, I. Franca, K. Kurtenbach, S. M. Schafer, L. Vitorino, L. Goncalves, S. Baptista, M. L. Vieira, and C. Cunha. 2004. First 
Isolation of Borrelia Iusitaniae from a Human Patient. J Clin Microbiol 42:13161318.

56. Comstock, L. E., and D. D. Thomas. 1991. Characterization of Borrelia burgdorferi invasion of cultured endothelial cells. Microb Pathog 10:137-48. 57. Comstock, L. E., and D. D. Thomas. 1989. Penetration of endothelial cell monolayers by Borrelia burgdorferi. Infect Immun 57:1626-8.

58. Concepcion, M. B., and D. R. Nelson. 2003. Expression of spoT in Borrelia burgdorferi during Serum Starvation. J Bacteriol 185:444-52.

59. Craven, R. C., and T. C. Montie. 1983. Chemotaxis of Pseudomonas aeruginosa: involvement of methylation. J Bacteriol 154:780-6.

60. Das, S., S. W. Barthold, S. S. Giles, R. R. Montgomery, S. R. Telford, 3rd, and E. Fikrig. 1997. Temporal pattern of Borrelia burgdorferi p21 expression in ticks and the mammalian host. J Clin Invest 99:987-95.

61. Davey, H. M., and D. B. Kell. 1996. Flow cytometry and cell sorting of heterogeneous microbial populations: the importance of single-cell analyses. Microbiol Rev 60:641-96.

62. De Martino, S. J., J. A. Carlyon, and E. Fikrig. 2001. Coinfection with Borrelia burgdorferi and the agent of human granulocytic ehrlichiosis. N Engl $\mathrm{J}$ Med 345:150-1.

63. Dong, Z., M. D. Edelstein, and L. J. Glickstein. 1997. CD8+ T cells are activated during the early Th1 and Th2 immune responses in a murine Lyme disease model. Infect Immun 65:5334-7.

64. Dressler, F., J. A. Whalen, B. N. Reinhardt, and A. C. Steere. 1993. Western blotting in the serodiagnosis of Lyme disease. J Infect Dis 167:392-400. 65. Dusenbery, D. B. 1998. Spatial sensing of stimulus gradients can be superior to temporal sensing for free-swimming bacteria. Biophys $\mathrm{J}$ 74:2272-7. 66. Dykhuizen, D. E., and G. Baranton. 2001. The implications of a low rate of horizontal transfer in Borrelia. Trends Microbiol 9:344-50.

67. Eggers, C. H., M. J. Caimano, M. L. Clawson, W. G. Miller, D. S. Samuels, and J. D. Radolf. 2002. Identification of loci critical for replication and compatibility of a Borrelia burgdorferi cp32 plasmid and use of a cp32-based 
shuttle vector for the expression of fluorescent reporters in the Lyme disease spirochaete. Mol Microbiol 43:281-95.

68. Eggers, C. H., B. J. Kimmel, J. L. Bono, A. F. Elias, P. Rosa, and D. S. Samuels. 2001. Transduction by phiBB-1, a Bacteriophage of Borrelia burgdorferi. J Bacteriol 183:4771-8.

69. Eggers, C. H., and D. S. Samuels. 1999. Molecular evidence for a new bacteriophage of Borrelia burgdorferi. J Bacteriol 181:7308-13.

70. Elias, A., J. L. Bono, K. Tilly, and P. Rosa. 1998. Growth of infectious and non-infectious $B$. burgdorferi at different salt concentrations. Wien Klin Wochenschr 110:863-5.

71. Falke, J. J., R. B. Bass, S. L. Butler, S. A. Chervitz, and M. A. Danielson. 1997. The two-component signaling pathway of bacterial chemotaxis: a molecular view of signal transduction by receptors, kinases, and adaptation enzymes. Annu Rev Cell Dev Biol 13:457-512.

72. Falke, J. J., and G. L. Hazelbauer. 2001. Transmembrane signaling in bacterial chemoreceptors. Trends Biochem Sci 26:257-65.

73. Ferrandez, A., A. C. Hawkins, D. T. Summerfield, and C. S. Harwood. 2002. Cluster II che genes from Pseudomonas aeruginosa are required for an optimal chemotactic response. J Bacteriol 184:4374-83.

74. Fosnaugh, K., and E. P. Greenberg. 1988. Motility and chemotaxis of Spirochaeta aurantia: computer-assisted motion analysis. J Bacteriol 170:176874.

75. Frank, K. L., S. F. Bundle, M. E. Kresge, C. H. Eggers, and D. S. Samuels. 2003. aadA confers streptomycin resistance in Borrelia burgdorferi. J Bacteriol 185:6723-7.

76. Fraser, C. M., S. Casjens, W. M. Huang, G. G. Sutton, R. Clayton, R. Lathigra, O. White, K. A. Ketchum, R. Dodson, E. K. Hickey, M. Gwinn, B. Dougherty, J. F. Tomb, R. D. Fleischmann, D. Richardson, J. Peterson, A. R. Kerlavage, J. Quackenbush, S. Salzberg, M. Hanson, R. van Vugt, N. Palmer, M. D. Adams, J. Gocayne, J. C. Venter, and et al. 1997. Genomic sequence of a Lyme disease spirochaete, Borrelia burgdorferi. Nature 390:580-6. 
77. Fraser, C. M., S. J. Norris, G. M. Weinstock, O. White, G. G. Sutton, R. Dodson, M. Gwinn, E. K. Hickey, R. Clayton, K. A. Ketchum, E. Sodergren, J. M. Hardham, M. P. McLeod, S. Salzberg, J. Peterson, H. Khalak, D. Richardson, J. K. Howell, M. Chidambaram, T. Utterback, L. McDonald, P. Artiach, C. Bowman, M. D. Cotton, J. C. Venter, and et al. 1998. Complete genome sequence of Treponema pallidum, the syphilis spirochete. Science 281:375-88.

78. Ge, Y., C. Li, L. Corum, C. A. Slaughter, and N. W. Charon. 1998. Structure and expression of the FlaA periplasmic flagellar protein of Borrelia burgdorferi. J Bacteriol 180:2418-25.

79. Ge, Y., I. G. Old, I. Saint Girons, and N. W. Charon. 1997. Molecular characterization of a large Borrelia burgdorferi motility operon which is initiated by a consensus sigma70 promoter. J Bacteriol 179:2289-99.

80. Ge, Y. a. C., N.W. 1997. An Unexpected flaA Homolog Is Present and Expressed in Borrelia burgdorferi. J Bacteriol 179:552-556.

81. Ge, Y. a. C., Nyles. 1997. Molecular characterization of a flagellar/chemotaxis opernon in the spriochete Borrelia burgdorferi. FEMS Microbiol Lett 152:425-431.

82. Ge, Y. a. N. W. C. 1997. Identification of a large motility operon in Borrelia burgdorferi by semi-random PCR chromosome walking. Gene 189:195-201.

83. Gebbia, J. A., J. L. Coleman, and J. L. Benach. 2001. Borrelia spirochetes upregulate release and activation of matrix metalloproteinase gelatinase $B$ (MMP-9) and collagenase 1 (MMP-1) in human cells. Infect Immun 69:456-62.

84. Gebbia, J. A., J. L. Coleman, and J. L. Benach. 2004. Selective induction of matrix metalloproteinases by Borrelia burgdorferi via toll-like receptor 2 in monocytes. J Infect Dis 189:113-9.

85. Gegner, J. A., D. R. Graham, A. F. Roth, and F. W. Dahlquist. 1992. Assembly of an MCP receptor, CheW, and kinase CheA complex in the bacterial chemotaxis signal transduction pathway. Cell 70:975-82.

86. Gern, L., A. Estrada-Peäna, F. Frandsen, J. S. Gray, T. G. Jaenson, F. Jongejan, O. Kahl, E. Korenberg, R. Mehl, and P. A. Nuttall. 1998. European 
reservoir hosts of Borrelia burgdorferi sensu lato. Zentralbl Bakteriol 287:196204.

87. Gestwicki, J. E., A. C. Lamanna, R. M. Harshey, L. L. McCarter, L. L. Kiessling, and J. Adler. 2000. Evolutionary conservation of methyl-accepting chemotaxis protein location in Bacteria and Archaea. J Bacteriol 182:6499-502. 88. Gilmore, R. D., Jr., M. L. Mbow, and B. Stevenson. 2001. Analysis of Borrelia burgdorferi gene expression during life cycle phases of the tick vector Ixodes scapularis. Microbes Infect 3:799-808.

89. Goldstein, S. F., K. F. Buttle, and N. W. Charon. 1996. Structural analysis of the Leptospiraceae and Borrelia burgdorferi by high-voltage electron microscopy. J Bacteriol 178:6539-45.

90. Goldstein, S. F., and N. W. Charon. 1990. Multiple-exposure photographic analysis of a motile spirochete. Proc Natl Acad Sci U S A 87:4895-9.

91. Goldstein, S. F., N. W. Charon, and J. A. Kreiling. 1994. Borrelia burgdorferi swims with a planar waveform similar to that of eukaryotic flagella. Proc Natl Acad Sci U S A 91:3433-7.

92. Gosink, K. K., R. Kobayashi, I. Kawagishi, and C. C. Hase. 2002. Analyses of the roles of the three cheA homologs in chemotaxis of Vibrio cholerae. J Bacteriol 184:1767-71.

93. Goulbourne, E. A., Jr., and E. P. Greenberg. 1981. Chemotaxis of Spirochaeta aurantia: involvement of membrane potential in chemosensory signal transduction. J Bacteriol 148:837-44.

94. Goy, M. F., M. S. Springer, and J. Adler. 1977. Sensory transduction in Escherichia coli: role of a protein methylation reaction in sensory adaptation. Proc Natl Acad Sci U S A 74:4964-8.

95. Greenberg, E. P., and E. Canale-Parola. 1977. Chemotaxis in Spirochaeta aurantia. J Bacteriol 130:485-94.

96. Greenberg, E. P., and E. Canale-Parola. 1977. Relationship between cell coiling and motility of spirochetes in viscous environments. J Bacteriol 131:960-9. 
97. Grimm, A. C., and C. S. Harwood. 1997. Chemotaxis of Pseudomonas spp. to the polyaromatic hydrocarbon naphthalene. Appl Environ Microbiol 63:4111-5.

98. Gunasekera, T. S., P. V. Attfield, and D. A. Veal. 2000. A flow cytometry method for rapid detection and enumeration of total bacteria in milk. Appl Environ Microbiol 66:1228-32.

99. Hamblin, P. A., N. A. Bourne, and J. P. Armitage. 1997. Characterization of the chemotaxis protein CheW from Rhodobacter sphaeroides and its effect on the behaviour of Escherichia coli. Mol Microbiol 24:41-51.

100. Hang, L., M. John, M. Asaduzzaman, E. A. Bridges, C. Vanderspurt, T. J. Kirn, R. K. Taylor, J. D. Hillman, A. Progulske-Fox, M. Handfield, E. T. Ryan, and S. B. Calderwood. 2003. Use of in vivo-induced antigen technology (IVIAT) to identify genes uniquely expressed during human infection with Vibrio cholerae. Proc Natl Acad Sci U S A 100:8508-13.

101. Hanson, M. S., N. K. Patel, D. R. Cassatt, and N. D. Ulbrandt. 2000. Evidence for vaccine synergy between Borrelia burgdorferi decorin binding protein $A$ and outer surface protein $A$ in the mouse model of Lyme borreliosis. Infect Immun 68:6457-60.

102. Hellwage, J., T. Meri, T. Heikkila, A. Alitalo, J. Panelius, P. Lahdenne, I. J. Seppala, and S. Meri. 2001. The complement regulator factor $\mathrm{H}$ binds to the surface protein OspE of Borrelia burgdorferi. J Biol Chem 276:8427-35.

103. Helmann, J. D. 1991. Alternative sigma factors and the regulation of flagellar gene expression. Mol Microbiol 5:2875-82.

104. Hirschman, A., M. Boukhvalova, R. VanBruggen, A. J. Wolfe, and R. C. Stewart. 2001. Active site mutations in CheA, the signal-transducing protein kinase of the chemotaxis system in Escherichia coli. Biochemistry 40:13876-87. 105. Hobson, G. 2000. President of Hobson Tracking Ltd. In R. Bakker (ed.), Morgantown, WV.

106. Hoefel, D., W. L. Grooby, P. T. Monis, S. Andrews, and C. P. Saint. 2003. Enumeration of water-borne bacteria using viability assays and flow cytometry: a comparison to culture-based techniques. J Microbiol Methods 55:585-97. 
107. Holt, S. C. 1978. Anatomy and chemistry of spirochetes. Microbiol Rev 42:114-60.

108. Hubner, A., X. Yang, D. M. Nolen, T. G. Popova, F. C. Cabello, and M. V. Norgard. 2001. Expression of Borrelia burgdorferi OspC and DbpA is controlled by a RpoN-RpoS regulatory pathway. Proc Natl Acad Sci U S A 98:12724-9. 109. Hudson, C. R., J. G. Frye, F. D. Quinn, and F. C. Gherardini. 2001. Increased expression of Borrelia burgdorferi vlsE in response to human endothelial cell membranes. Mol Microbiol 41:229-39.

110. Hundhausen, C., D. Misztela, T. A. Berkhout, N. Broadway, P. Saftig, K. Reiss, D. Hartmann, F. Fahrenholz, R. Postina, V. Matthews, K. J. Kallen, S. Rose-John, and A. Ludwig. 2003. The disintegrin-like metalloproteinase ADAM10 is involved in constitutive cleavage of CX3CL1 (fractalkine) and regulates CX3CL1-mediated cell-cell adhesion. Blood 102:1186-95.

111. Indest, K. J., J. K. Howell, M. B. Jacobs, D. Scholl-Meeker, S. J. Norris, and M. T. Philipp. 2001. Analysis of Borrelia burgdorferi vlsE Gene Expression and Recombination in the Tick Vector. Infect Immun 69:7083-90.

112. Jasuja, R., J. Keyoung, G. P. Reid, D. R. Trentham, and S. Khan. 1999. Chemotactic responses of Escherichia coli to small jumps of photoreleased Laspartate. Biophys J 76:1706-19.

113. Jeffery Walker, D. T. 1988. Photolabile 1-(2-Nitrophenyl)ethyl Phosphate Esters of Adenine Nucleotide Analogues. Synthesis and Mechanism of Photolysis. Journal of the American Chemical Society 110:7170-7177.

114. Jensen, T. K., M. Boye, P. Ahrens, B. Korsager, P. S. Teglbjaerg, C. F. Lindboe, and K. Moller. 2001. Diagnostic Examination of Human Intestinal Spirochetosis by Fluorescent In Situ Hybridization for Brachyspira aalborgi, Brachyspira pilosicoli, and Other Species of the Genus Brachyspira (Serpulina). J Clin Microbiol 39:4111-8.

115. Jespersen, D. J., T. F. Smith, J. E. Rosenblatt, and I. F. Cockerill. 2002. Comparison of the Borrelia DotBlot G, MarDx, and VIDAS Enzyme Immunoassays for Detecting Immunoglobulin G Antibodies to Borrelia burgdorferi in Human Serum. J Clin Microbiol 40:4782-4. 
116. Johnson, R. C., Schmid, G.P., Hyde, F.W., Steigerwalt, A.G., and Brenner, D.J. 1984. Borrelia burgdorferi sp. nov.: Etiologic Agent of Lyme Disease. International Systematic Bacteriology:496-497.

117. Johnson, S. E., G. C. Klein, G. P. Schmid, and J. C. Feeley. 1984.

Susceptibility of the Lyme disease spirochete to seven antimicrobial agents. Yale J Biol Med 57:549-53.

118. Josenhans, C., and S. Suerbaum. 2002. The role of motility as a virulence factor in bacteria. Int J Med Microbiol 291:605-14.

119. Karim, Q. N., R. P. Logan, J. Puels, A. Karnholz, and M. L. Worku. 1998. Measurement of motility of Helicobacter pylori, Campylobacter jejuni, and Escherichia coli by real time computer tracking using the Hobson BacTracker. J Clin Pathol 51:623-8.

120. Karlinsey, J. E., J. Lonner, K. L. Brown, and K. T. Hughes. 2000.

Translation/secretion coupling by type III secretion systems. Cell 102:487-97.

121. Kataoka, M., H. Li, S. Arakawa, and H. Kuramitsu. 1997. Characterization of a methyl-accepting chemotaxis protein gene, dmcA, from the oral spirochete Treponema denticola. Infect Immun 65:4011-6.

122. Kathariou, S., and E. P. Greenberg. 1983. Chemoattractants elicit methylation of specific polypeptides in Spirochaeta aurantia. J Bacteriol 156:95100.

123. Kehry, M. R., T. G. Doak, and F. W. Dahlquist. 1985. Sensory adaptation in bacterial chemotaxis: regulation of demethylation. J Bacteriol 163:983-90.

124. Kelly-Wintenberg, K., and T. C. Montie. 1994. Chemotaxis to oligopeptides by Pseudomonas aeruginosa. Appl Environ Microbiol 60:363-7.

125. Kennedy, M. J., E. L. Rosey, and R. J. Yancey, Jr. 1997. Characterization of flaA- and flaB- mutants of Serpulina hyodysenteriae: both flagellin subunits, FlaA and FlaB, are necessary for full motility and intestinal colonization. FEMS Microbiol Lett 153:119-28.

126. Kennedy, M. J., and R. J. Yancey, Jr. 1996. Motility and chemotaxis in Serpulina hyodysenteriae. Vet Microbiol 49:21-30. 
127. Kèorner, M., and J. O. Gebbers. 2003. Clinical significance of human intestinal spirochetosis-a morphologic approach. Infection 31:341-9.

128. Keyhani, N. O., L. X. Wang, Y. C. Lee, and S. Roseman. 2000. The chitin disaccharide, N,N'-diacetylchitobiose, is catabolized by Escherichia coli and is transported/phosphorylated by the phosphoenolpyruvate:glycose phosphotransferase system. J Biol Chem 275:33084-90.

129. Khan, S., J. L. Spudich, J. A. McCray, and D. R. Trentham. 1995. Chemotactic signal integration in bacteria. Proc Natl Acad Sci U S A 92:9757-61. 130. Kim, C., M. Jackson, R. Lux, and S. Khan. 2001. Determinants of chemotactic signal amplification in Escherichia coli. J Mol Biol 307:119-35. 131. Kimsey, R. B., and A. Spielman. 1990. Motility of Lyme disease spirochetes in fluids as viscous as the extracellular matrix. J Infect Dis 162:12058.

132. King, M. W. Tuesday, 12-Aug-2003 20:02:03 EST 2003, posting date. Glycosaminoglycans and Proteoglycans http://www.indstate.edu/thcme/mwking/glycans.html. [Online.] 133. Klempner, M. S., L. T. Hu, J. Evans, C. H. Schmid, G. M. Johnson, R. P. Trevino, D. Norton, L. Levy, D. Wall, J. McCall, M. Kosinski, and A. Weinstein. 2001. Two controlled trials of antibiotic treatment in patients with persistent symptoms and a history of Lyme disease. N Engl J Med 345:85-92.

134. Kort, E. N., M. F. Goy, S. H. Larsen, and J. Adler. 1975. Methylation of a membrane protein involved in bacterial chemotaxis. Proc Natl Acad Sci U S A 72:3939-43.

135. Labandeira-Rey, M., J. Seshu, and J. T. Skare. 2003. The absence of linear plasmid 25 or 28-1 of Borrelia burgdorferi dramatically alters the kinetics of experimental infection via distinct mechanisms. Infect Immun 71:4608-13. 136. Larsen, S. H., J. Adler, J. J. Gargus, and R. W. Hogg. 1974. Chemomechanical coupling without ATP: the source of energy for motility and chemotaxis in bacteria. Proc Natl Acad Sci U S A 71:1239-43. 
137. Larsen, S. H., R. W. Reader, E. N. Kort, W. W. Tso, and J. Adler. 1974. Change in direction of flagellar rotation is the basis of the chemotactic response in Escherichia coli. Nature 249:74-7.

138. Le Fleche, A., D. Postic, K. Girardet, O. Peter, and G. Baranton. 1997. Characterization of Borrelia lusitaniae sp. nov. by $16 \mathrm{~S}$ ribosomal DNA sequence analysis. Int J Syst Bacteriol 47:921-5.

139. Lebaron, P., N. Parthuisot, and P. Catala. 1998. Comparison of blue nucleic acid dyes for flow cytometric enumeration of bacteria in aquatic systems. Appl Environ Microbiol 64:1725-30.

140. Lebech, A. M., K. Hansen, F. Brandrup, O. Clemmensen, and L. Halkier$S$ 'rensen. 2000. Diagnostic value of PCR for detection of Borrelia burgdorferi DNA in clinical specimens from patients with erythema migrans and Lyme neuroborreliosis. Mol Diagn 5:139-50.

141. Lee, S. H., S. M. Butler, and A. Camilli. 2001. Selection for in vivo regulators of bacterial virulence. Proc Natl Acad Sci U S A 98:6889-94. 142. Levin, M. D., T. S. Shimizu, and D. Bray. 2002. Binding and diffusion of CheR molecules within a cluster of membrane receptors. Biophys J 82:1809-17. 143. Levine, J. F., M. L. Wilson, and A. Spielman. 1985. Mice as reservoirs of the Lyme disease spirochete. Am J Trop Med Hyg 34:355-60.

144. Li, C., R. G. Bakker, M. A. Motaleb, M. L. Sartakova, F. C. Cabello, and N. W. Charon. 2002. Asymmetrical flagellar rotation in Borrelia burgdorferi nonchemotactic mutants. Proc Natl Acad Sci U S A 99:6169-74.

145. Li, C., L. Corum, D. Morgan, E. L. Rosey, T. B. Stanton, and N. W. Charon. 2000. The spirochete FlaA periplasmic flagellar sheath protein impacts flagellar helicity. J Bacteriol 182:6698-706.

146. Li, C., A. Motaleb, M. Sal, S. F. Goldstein, and N. W. Charon. 2000.

Spirochete periplasmic flagella and motility. J Mol Microbiol Biotechnol 2:345-54.

147. Li, H., S. Arakawa, Q. D. Deng, and H. Kuramitsu. 1999. Characterization of a novel methyl-accepting chemotaxis gene, $d m c B$, from the oral spirochete Treponema denticola. Infect Immun 67:694-9. 
148. Li, R. C., S. W. Lee, and J. S. Lam. 1996. Novel method for assessing postantibiotic effect by using the Coulter counter. Antimicrob Agents Chemother 40:1751-3.

149. Liang, F. T., A. L. Alvarez, Y. Gu, J. M. Nowling, R. Ramamoorthy, and M. T. Philipp. 1999. An immunodominant conserved region within the variable domain of VIsE, the variable surface antigen of Borrelia burgdorferi. J Immunol 163:5566-73.

150. Liang, F. T., M. B. Jacobs, L. C. Bowers, and M. T. Philipp. 2002. An immune evasion mechanism for spirochetal persistence in Lyme borreliosis. $\mathrm{J}$ Exp Med 195:415-22.

151. Lin, B., S. A. Short, M. Eskildsen, M. S. Klempner, and L. T. Hu. 2001. Functional testing of putative oligopeptide permease (Opp) proteins of Borrelia burgdorferi: a complementation model in opp(-) Escherichia coli. Biochim Biophys Acta 1499:222-31.

152. Lux, R., K. Jahreis, K. Bettenbrock, J. S. Parkinson, and J. W. Lengeler. 1995. Coupling the phosphotransferase system and the methyl-accepting chemotaxis protein-dependent chemotaxis signaling pathways of Escherichia coli. Proc Natl Acad Sci U S A 92:11583-7.

153. Lux, R., J. N. Miller, N. H. Park, and W. Shi. 2001. Motility and chemotaxis in tissue penetration of oral epithelial cell layers by Treponema denticola. Infect Immun 69:6276-83.

154. Lux, R., A. Moter, and W. Shi. 2000. Chemotaxis in pathogenic spirochetes: directed movement toward targeting tissues? J Mol Microbiol Biotechnol 2:355-64.

155. Lux, R., V. R. Munasinghe, F. Castellano, J. W. Lengeler, J. E. Corrie, and S. Khan. 1999. Elucidation of a PTS-carbohydrate chemotactic signal pathway in Escherichia coli using a time-resolved behavioral assay. Mol Biol Cell 10:113346.

156. Lux, R., J. H. Sim, J. P. Tsai, and W. Shi. 2002. Construction and characterization of a cheA mutant of Treponema denticola. J Bacteriol 184:31304. 
157. Lybarger, S. R., and J. R. Maddock. 2000. Differences in the polar clustering of the high- and low-abundance chemoreceptors of Escherichia coli. Proc Natl Acad Sci U S A 97:8057-62.

158. Lybarger, S. R., and J. R. Maddock. 2001. Polarity in action: asymmetric protein localization in bacteria. J Bacteriol 183:3261-7.

159. Macnab, R. M., and D. E. Koshland, Jr. 1972. The gradient-sensing mechanism in bacterial chemotaxis. Proc Natl Acad Sci U S A 69:2509-12. 160. Magariyama, Y., S. Sugiyama, and S. Kudo. 2001. Bacterial swimming speed and rotation rate of bundled flagella. FEMS Microbiol Lett 199:125-9. 161. Manson, M. D., V. Blank, G. Brade, and C. F. Higgins. 1986. Peptide chemotaxis in E. coli involves the Tap signal transducer and the dipeptide permease. Nature 321:253-6.

162. Manweiler, S. A., R. S. Lane, and C. H. Tempelis. 1992. The western fence lizard Sceloporus occidentalis: evidence of field exposure to Borrelia burgdorferi in relation to infestation by Ixodes pacificus (Acari: Ixodidae). Am J Trop Med Hyg 47:328-36.

163. Martin, A. C., U. Nair, J. P. Armitage, and J. R. Maddock. 2003. Polar localization of CheA2 in Rhodobacter sphaeroides requires specific Che homologs. J Bacteriol 185:4667-71.

164. Martin, A. C., G. H. Wadhams, and J. P. Armitage. 2001. The roles of the multiple CheW and CheA homologues in chemotaxis and in chemoreceptor localization in Rhodobacter sphaeroides. Mol Microbiol 40:1261-72.

165. Masuzawa, T., N. Takada, M. Kudeken, T. Fukui, Y. Yano, F. Ishiguro, Y. Kawamura, Y. Imai, and T. Ezaki. 2001. Borrelia sinica sp. nov., a Lyme diseaserelated Borrelia species isolated in China. Int J Syst Evol Microbiol 51:1817-24. 166. Matuschka, F. R., P. Fischer, M. Heiler, D. Richter, and A. Spielman. 1992. Capacity of European animals as reservoir hosts for the Lyme disease spirochete. J Infect Dis 165:479-83.

167. Matuschka, F. R., and A. Spielman. 1986. The emergence of Lyme disease in a changing environment in North America and central Europe. Exp Appl Acarol 2:337-53. 
168. Mazumder, R., T. J. Phelps, N. R. Krieg, and R. E. Benoit. 1999.

Determining chemotactic responses by two subsurface microaerophiles using a simplified capillary assay method. J Microbiol Methods 37:255-63.

169. McDowell, J. V., S. Y. Sung, L. T. Hu, and R. T. Marconi. 2002. Evidence that the variable regions of the central domain of VIsE are antigenic during infection with Lyme disease spirochetes. Infect Immun 70:4196-203.

170. Merrell, D. S., S. M. Butler, F. Qadri, N. A. Dolganov, A. Alam, M. B. Cohen, S. B. Calderwood, G. K. Schoolnik, and A. Camilli. 2002. Host-induced epidemic spread of the cholera bacterium. Nature 417:642-5.

171. Mesibov, R., and J. Adler. 1972. Chemotaxis toward amino acids in Escherichia coli. J Bacteriol 112:315-26.

172. Mesibov, R., G. W. Ordal, and J. Adler. 1973. The range of attractant concentrations for bacterial chemotaxis and the threshold and size of response over this range. Weber law and related phenomena. J Gen Physiol 62:203-23. 173. Meyer, A. L., C. Trollmo, F. Crawford, P. Marrack, A. C. Steere, B. T. Huber, J. Kappler, and D. A. Hafler. 2000. Direct enumeration of Borrelia-reactive CD4 T cells ex vivo by using MHC class II tetramers. Proc Natl Acad Sci U S A 97:11433-8.

174. Montgomery, R. R., D. Lusitani, A. de Boisfleury Chevance, and S. E. Malawista. 2002. Human phagocytic cells in the early innate immune response to Borrelia burgdorferi. J Infect Dis 185:1773-9.

175. Montgomery, R. R., S. E. Malawista, K. J. Feen, and L. K. Bockenstedt. 1996. Direct demonstration of antigenic substitution of Borrelia burgdorferi ex vivo: exploration of the paradox of the early immune response to outer surface proteins $A$ and $C$ in Lyme disease. J Exp Med 183:261-9.

176. Motaleb, M. A., L. Corum, J. L. Bono, A. F. Elias, P. Rosa, D. S. Samuels, and N. W. Charon. 2000. Borrelia burgdorferi periplasmic flagella have both skeletal and motility functions. Proc Natl Acad Sci U S A 97:10899-904. 177. Moulton, R. C., and T. C. Montie. 1979. Chemotaxis by Pseudomonas aeruginosa. J Bacteriol 137:274-80. 
178. Nadelman, R. B., J. Nowakowski, D. Fish, R. C. Falco, K. Freeman, D. McKenna, P. Welch, R. Marcus, M. E. Aguero-Rosenfeld, D. T. Dennis, and G. P. Wormser. 2001. Prophylaxis with single-dose doxycycline for the prevention of Lyme disease after an Ixodes scapularis tick bite. N Engl J Med 345:79-84. 179. Nadelman, R. B., and G. P. Wormser. 1998. Lyme borreliosis. Lancet 352:557-65.

180. Narasimhan, S., F. Santiago, R. A. Koski, B. Brei, J. F. Anderson, D. Fish, and E. Fikrig. 2002. Examination of the Borrelia burgdorferi transcriptome in Ixodes scapularis during feeding. J Bacteriol 184:3122-5.

181. Ninfa, E. G., A. Stock, S. Mowbray, and J. Stock. 1991. Reconstitution of the bacterial chemotaxis signal transduction system from purified components. $J$ Biol Chem 266:9764-70.

182. Nyberg, W. L. 1975. Intermediate Biophysical Mechanics. Benjamin Cummings, San Francisco.

183. Obonyo, M., U. G. Munderloh, T. N. Sam, and T. J. Kurtti. 2002.

Cultivation at 37 degrees $C$ enhances Borrelia burgdorferi sensu stricto infectivity for hamsters. Med Microbiol Immunol (Berl) 191:33-9.

184. Ojaimi, C., C. Brooks, S. Casjens, P. Rosa, A. Elias, A. Barbour, A. Jasinskas, J. Benach, L. Katona, J. Radolf, M. Caimano, J. Skare, K. Swingle, D. Akins, and I. Schwartz. 2003. Profiling of Temperature-Induced Changes in Borrelia burgdorferi Gene Expression by Using Whole Genome Arrays. Infect Immun 71:1689-1705.

185. Oliver, J. H., Jr., T. Lin, L. Gao, K. L. Clark, C. W. Banks, L. A. Durden, A. M. James, and F. W. Chandler, Jr. 2003. An enzootic transmission cycle of Lyme borreliosis spirochetes in the southeastern United States. Proc Natl Acad Sci U S A 100:11642-5.

186. Olsâen, B., T. G. Jaenson, and S. Bergstrèom. 1995. Prevalence of Borrelia burgdorferi sensu lato-infected ticks on migrating birds. Appl Environ Microbiol 61:3082-7.

187. Olsâen, B., T. G. Jaenson, L. Noppa, J. Bunikis, and S. Bergstrèom. 1993. A Lyme borreliosis cycle in seabirds and Ixodes uriae ticks. Nature 362:340-2. 
188. Olsen, B., D. C. Duffy, T. G. Jaenson, A. Gylfe, J. Bonnedahl, and S. Bergstrèom. 1995. Transhemispheric exchange of Lyme disease spirochetes by seabirds. J Clin Microbiol 33:3270-4.

189. Ordal, G. W., D. P. Villani, and M. S. Rosendahl. 1979. Chemotaxis towards sugars by Bacillus subtilis. J Gen Microbiol 115:167-72.

190. Ostberg, Y. 2002. Functional Analysis of the Membrane Associated Proteins P 13, CtpA, and bbMGS from Borrelia burgdorferi Spirochetes. Doctorial. Umea University, Umea.

191. Pachner, A. R., and A. C. Steere. 1984. Neurological findings of Lyme disease. Yale J Biol Med 57:481-3.

192. Pal, U., A. M. de Silva, R. R. Montgomery, D. Fish, J. Anguita, J. F. Anderson, Y. Lobet, and E. Fikrig. 2000. Attachment of Borrelia burgdorferi within Ixodes scapularis mediated by outer surface protein A. J Clin Invest 106:561-9. 193. Pal, U., X. Yang, M. Chen, L. K. Bockenstedt, J. F. Anderson, R. A. Flavell, M. V. Norgard, and E. Fikrig. 2004. OspC facilitates Borrelia burgdorferi invasion of Ixodes scapularis salivary glands. J Clin Invest 113:220-30.

194. Parkinson, J. S. 1977. Behavioral genetics in bacteria. Annu Rev Genet 11:397-414.

195. Parkinson, J. S., and S. E. Houts. 1982. Isolation and behavior of Escherichia coli deletion mutants lacking chemotaxis functions. J Bacteriol 151:106-13.

196. Parveen, N., M. Caimano, J. D. Radolf, and J. M. Leong. 2003. Adaptation of the Lyme disease spirochete to the mammalian host environment results in enhanced glycosaminoglycan and host cell binding. Mol Microbiol 47:1433-44. 197. Paster, B. J., and F. E. Dewhirst. 2000. Phylogenetic foundation of spirochetes. J Mol Microbiol Biotechnol 2:341-4.

198. Pereira, M., J. A. Parente, L. A. Bataus, D. D. Cardoso, R. B. Soares, and C. M. Soares. 2004. Chemotaxis and flagellar genes of Chromobacterium violaceum. Genet Mol Res 3:92-101.

199. Perriere, G., and J. Thioulouse. 2002. Use and misuse of correspondence analysis in codon usage studies. Nucleic Acids Res 30:4548-55. 
200. Peters, S. R., M. Valdez, G. Riviere, and D. D. Thomas. 1999. Adherence to and penetration through endothelial cells by oral treponemes. Oral Microbiol Immunol 14:379-83.

201. Petrino, M. G., and R. N. Doetsch. 1978. 'Viscotaxis', a new behavioural response of Leptospira interrogans (biflexa) strain B16. J Gen Microbiol 109:1137.

202. Picken, R. N., Y. Cheng, F. Strle, and M. M. Picken. 1996. Patient isolates of Borrelia burgdorferi sensu lato with genotypic and phenotypic similarities of strain 25015. J Infect Dis 174:1112-5.

203. Piesman, J. 1993. Dynamics of Borrelia burgdorferi transmission by nymphal Ixodes dammini ticks. J Infect Dis 167:1082-5.

204. Piesman, J., T. N. Mather, R. J. Sinsky, and A. Spielman. 1987. Duration of tick attachment and Borrelia burgdorferi transmission. J Clin Microbiol 25:5578.

205. Pohl-Koppe, A., A. Kaunicnik, and B. Wilske. 2001. Characterization of the cellular and humoral immune response to outer surface protein $\mathrm{C}$ and outer surface protein 17 in children with early disseminated Lyme borreliosis. Med Microbiol Immunol (Berl) 189:193-200.

206. Poland, G. A. 2001. Prevention of Lyme disease: a review of the evidence. Mayo Clin Proc 76:713-24.

207. Porter, S. L., A. V. Warren, A. C. Martin, and J. P. Armitage. 2002. The third chemotaxis locus of Rhodobacter sphaeroides is essential for chemotaxis. Mol Microbiol 46:1081-94.

208. Postic, D., N. M. Ras, R. S. Lane, M. Hendson, and G. Baranton. 1998. Expanded diversity among Californian borrelia isolates and description of Borrelia bissettii sp. nov. (formerly Borrelia group DN127). J Clin Microbiol 36:3497-504.

209. Putschky, N., S. Schnarr, J. Wollenhaupt, H. Zeidler, and J. G. Kuipers. 2001. Intra-articular co-infection by Borrelia burgdorferi and Chlamydia trachomatis. Ann Rheum Dis 60:632-4. 
210. Rauer, S., and A. Conrad. 2001. Competitive inhibition ELISA for the detection of Borrelia burgdorferi antigens--failure to detect antigen in the cerebrospinal fluid from patients with neuroborreliosis. J Med Microbiol 50:577-8. 211. Ren, S. X., G. Fu, X. G. Jiang, R. Zeng, Y. G. Miao, H. Xu, Y. X. Zhang, H. Xiong, G. Lu, L. F. Lu, H. Q. Jiang, J. Jia, Y. F. Tu, J. X. Jiang, W. Y. Gu, Y. Q. Zhang, Z. Cai, H. H. Sheng, H. F. Yin, Y. Zhang, G. F. Zhu, M. Wan, H. L. Huang, Z. Qian, S. Y. Wang, W. Ma, Z. J. Yao, Y. Shen, B. Q. Qiang, Q. C. Xia, X. K. Guo, A. Danchin, I. Saint Girons, R. L. Somerville, Y. M. Wen, M. H. Shi, Z. Chen, J. G. Xu, and G. P. Zhao. 2003. Unique physiological and pathogenic features of Leptospira interrogans revealed by whole-genome sequencing. Nature 422:888-93.

212. Rendulic, S., P. Jagtap, A. Rosinus, M. Eppinger, C. Baar, C. Lanz, H. Keller, C. Lambert, K. J. Evans, A. Goesmann, F. Meyer, R. E. Sockett, and S. C. Schuster. 2004. A predator unmasked: life cycle of Bdellovibrio bacteriovorus from a genomic perspective. Science 303:689-92.

213. Revel, A. T., A. M. Talaat, and M. V. Norgard. 2002. DNA microarray analysis of differential gene expression in Borrelia burgdorferi, the Lyme disease spirochete. Proc Natl Acad Sci U S A 99:1562-7.

214. Roberts, D. M., M. Caimano, J. McDowell, M. Theisen, A. Holm, E. Orff, D. Nelson, S. Wikel, J. Radolf, and R. T. Marconi. 2002. Environmental Regulation and Differential Production of Members of the Bdr Protein Family of Borrelia burgdorferi. Infect Immun 70:7033-7041.

215. Rosa, P., D. S. Samuels, D. Hogan, B. Stevenson, S. Casjens, and K. Tilly. 1996. Directed insertion of a selectable marker into a circular plasmid of Borrelia burgdorferi. J Bacteriol 178:5946-53.

216. Rosey, E. L., M. J. Kennedy, and R. J. Yancey, Jr. 1996. Dual flaA1 flaB1 mutant of Serpulina hyodysenteriae expressing periplasmic flagella is severely attenuated in a murine model of swine dysentery. Infect Immun 64:4154-62.

217. Rossler, D., H. Eiffert, S. Jauris-Heipke, G. Lehnert, V. Preac-Mursic, J. Teepe, T. Schlott, E. Soutschek, and B. Wilske. 1995. Molecular and 
immunological characterization of the $\mathrm{p} 83 / 100$ protein of various Borrelia burgdorferi sensu lato strains. Med Microbiol Immunol (Berl) 184:23-32. 218. Ruby, J. D., and N. W. Charon. 1998. Effect of temperature and viscosity on the motility of the spirochete Treponema denticola. FEMS Microbiol Lett 169:251-4.

219. Sadziene, A., P. A. Thompson, and A. G. Barbour. 1996. A flagella-less mutant of Borrelia burgdorferi as a live attenuated vaccine in the murine model of Lyme disease. J Infect Dis 173:1184-93.

220. Samuels, D. S. 1995. Electrotransformation of the Spirochete Borreali burgdorferi, p. 253. In J. A. Nickoloff (ed.), Methods of Molecular Biology, vol. Vol 47. Humana Press, Totowa NJ.

221. Sartakova, M., E. Dobrikova, and F. C. Cabello. 2000. Development of an extrachromosomal cloning vector system for use in Borrelia burgdorferi. Proc Natl Acad Sci U S A 97:4850-5.

222. Sartakova, M. L., E. Y. Dobrikova, M. A. Motaleb, H. P. Godfrey, N. W. Charon, and F. C. Cabello. 2001. Complementation of a nonmotile flaB mutant of Borrelia burgdorferi by chromosomal Integration of a plasmid containing a wildtype flaB allele. J Bacteriol 183:6558-64.

223. Sartakova, M. L., E. Y. Dobrikova, D. A. Terekhova, R. Devis, J. V. Bugrysheva, O. V. Morozova, H. P. Godfrey, and F. C. Cabello. 2003. Novel antibiotic-resistance markers in pGK12-derived vectors for Borrelia burgdorferi. Gene 303:131-7.

224. Scharf, B. 2002. Real-time imaging of fluorescent flagellar filaments of Rhizobium lupini $\mathrm{H} 13-3$ : flagellar rotation and $\mathrm{pH}$-induced polymorphic transitions. J Bacteriol 184:5979-86.

225. Schauder, S., K. Shokat, M. G. Surette, and B. L. Bassler. 2001. The LuxS family of bacterial autoinducers: biosynthesis of a novel quorum-sensing signal molecule. Mol Microbiol 41:463-76.

226. Schneider, W. R., Jr., and R. N. Doetsch. 1977. Temperature effects on bacterial movement. Appl Environ Microbiol 34:695-700. 
227. Schwan, T. G., J. Piesman, W. T. Golde, M. C. Dolan, and P. A. Rosa. 1995. Induction of an outer surface protein on Borrelia burgdorferi during tick feeding. Proc Natl Acad Sci U S A 92:2909-13.

228. Segall, J. E., M. D. Manson, and H. C. Berg. 1982. Signal processing times in bacterial chemotaxis. Nature 296:855-7.

229. Sellek, R. E., R. Escudero, H. Gil, I. Rodriguez, E. Chaparro, E. PerezPastrana, A. Vivo, and P. Anda. 2002. In Vitro Culture of Borrelia garinii Results in Loss of Flagella and Decreased Invasiveness. Infect Immun 70:4851-8.

230. Sèorensen, M., C. Lippuner, T. Kaiser, A. Misslitz, T. Aebischer, and D. Bumann. 2003. Rapidly maturing red fluorescent protein variants with strongly enhanced brightness in bacteria. FEBS Lett 552:110-4.

231. Seshadri, R., G. S. Myers, H. Tettelin, J. A. Eisen, J. F. Heidelberg, R. J. Dodson, T. M. Davidsen, R. T. DeBoy, D. E. Fouts, D. H. Haft, J. Selengut, Q. Ren, L. M. Brinkac, R. Madupu, J. Kolonay, S. A. Durkin, S. C. Daugherty, J. Shetty, A. Shvartsbeyn, E. Gebregeorgis, K. Geer, G. Tsegaye, J. Malek, B. Ayodeji, S. Shatsman, M. P. McLeod, D. Smajs, J. K. Howell, S. Pal, A. Amin, P. Vashisth, T. Z. McNeill, Q. Xiang, E. Sodergren, E. Baca, G. M. Weinstock, S. J. Norris, C. M. Fraser, and I. T. Paulsen. 2004. Comparison of the genome of the oral pathogen Treponema denticola with other spirochete genomes. Proc Natl Acad Sci U S A.

232. Shapiro, L., and R. Losick. 2000. Dynamic spatial regulation in the bacterial cell. Cell 100:89-98.

233. Shi, W. 1998. Chemotaxis in Borrelia burgdorferi. Journal of Bacteriology 180:231-35.

234. Shi, W., Z. Yang, Y. Geng, L. E. Wolinsky, and M. A. Lovett. 1998. Chemotaxis in Borrelia burgdorferi. J Bacteriol 180:231-5.

235. Shigematsu, M., Y. Meno, H. Misumi, and K. Amako. 1995. The measurement of swimming velocity of Vibrio cholerae and Pseudomonas aeruginosa using the video tracking methods. Microbiol Immunol 39:741-4. 
236. Shih, C. M., L. L. Chao, and C. P. Yu. 2002. Chemotactic migration of the Lyme disease spirochete (Borrelia burgdorferi) to salivary gland extracts of vector ticks. Am J Trop Med Hyg 66:616-21.

237. Shimizu, T. S., N. Le Novere, M. D. Levin, A. J. Beavil, B. J. Sutton, and D. Bray. 2000. Molecular model of a lattice of signalling proteins involved in bacterial chemotaxis. Nat Cell Biol 2:792-6.

238. Shoberg, R. J., and D. D. Thomas. 1993. Specific adherence of Borrelia burgdorferi extracellular vesicles to human endothelial cells in culture. Infect Immun 61:3892-900.

239. Sigal, L. H., and A. H. Tatum. 1988. Lyme disease patients' serum contains IgM antibodies to Borrelia burgdorferi that cross-react with neuronal antigens. Neurology 38:1439-42.

240. Sigal, L. H., J. M. Zahradnik, P. Lavin, S. J. Patella, G. Bryant, R. Haselby, E. Hilton, M. Kunkel, D. Adler-Klein, T. Doherty, J. Evans, P. J. Molloy, A. L. Seidner, J. R. Sabetta, H. J. Simon, M. S. Klempner, J. Mays, D. Marks, and S. E. Malawista. 1998. A vaccine consisting of recombinant Borrelia burgdorferi outer-surface protein A to prevent Lyme disease. Recombinant Outer-Surface Protein A Lyme Disease Vaccine Study Consortium. N Engl J Med 339:216-22. 241. Silberer, M., F. Koszik, G. Stingl, and E. Aberer. 2000. Downregulation of class II molecules on epidermal Langerhans cells in Lyme borreliosis. $\mathrm{Br} \mathrm{J}$ Dermatol 143:786-94.

242. Silverman, M., and M. Simon. 1977. Chemotaxis in Escherichia coli: methylation of che gene products. Proc Natl Acad Sci U S A 74:3317-21. 243. Sincock, S. A., and J. P. Robinson. 2001. Flow cytometric analysis of microorganisms, p. 511-37, Methods Cell Biology, vol. 64.

244. Slonczewski, J. L., R. M. Macnab, J. R. Alger, and A. M. Castle. 1982. Effects of $\mathrm{pH}$ and repellent tactic stimuli on protein methylation levels in Escherichia coli. J Bacteriol 152:384-99.

245. Sourjik, V., and H. C. Berg. 2002. Receptor sensitivity in bacterial chemotaxis. Proc Natl Acad Sci U S A 99:123-7. 
246. Sperandio, V., A. G. Torres, and J. B. Kaper. 2002. Quorum sensing Escherichia coli regulators $\mathrm{B}$ and $\mathrm{C}$ (QseBC): a novel two-component regulatory system involved in the regulation of flagella and motility by quorum sensing in $E$. coli. Mol Microbiol 43:809-21.

247. Springer, M. S., M. F. Goy, and J. Adler. 1979. Protein methylation in behavioural control mechanisms and in signal transduction. Nature 280:279-84. 248. Spudich, J. L., and D. E. Koshland, Jr. 1975. Quantitation of the sensory response in bacterial chemotaxis. Proc Natl Acad Sci U S A 72:710-3.

249. Stanek, G., and F. Strle. 2003. Lyme borreliosis. Lancet 362:1639-47.

250. Stavitsky, A. B. 1945. Studies on the Pathogenesis of Spirochetes. Journal of Bacteriology 76:179-192.

251. Steere, A. C. 1989. Lyme disease. N Engl J Med 321:586-96.

252. Steere, A. C., J. Coburn, and L. Glickstein. 2004. The emergence of Lyme disease. J Clin Invest 113:1093-101.

253. Steere, A. C., R. L. Grodzicki, A. N. Kornblatt, J. E. Craft, A. G. Barbour, W. Burgdorfer, G. P. Schmid, E. Johnson, and S. E. Malawista. 1983. The spirochetal etiology of Lyme disease. N Engl J Med 308:733-40.

254. Steere, A. C., D. Gross, A. L. Meyer, and B. T. Huber. 2001. Autoimmune mechanisms in antibiotic treatment-resistant Lyme arthritis. J Autoimmun 16:2638.

255. Steere, A. C., and S. E. Malawista. 1979. Cases of Lyme disease in the United States: locations correlated with distribution of Ixodes dammini. Ann Intern Med 91:730-3.

256. Steere, A. C., S. E. Malawista, D. R. Snydman, R. E. Shope, W. A. Andiman, M. R. Ross, and F. M. Steele. 1977. Lyme arthritis: an epidemic of oligoarticular arthritis in children and adults in three Connecticut communities. Arthritis Rheum 20:7-17.

257. Steere, A. C., G. McHugh, C. Suarez, J. Hoitt, N. Damle, and V. K.

Sikand. 2003. Prospective study of coinfection in patients with erythema migrans. Clin Infect Dis 36:1078-81. 
258. Stevenson, B., and K. Babb. 2002. LuxS-Mediated Quorum Sensing in Borrelia burgdorferi, the Lyme Disease Spirochete. Infect Immun 70:4099-105. 259. Stevenson, B., K. von Lackum, R. L. Wattier, J. D. McAlister, J. C. Miller, and K. Babb. 2003. Quorum sensing by the Lyme disease spirochete. Microbes Infect 5:991-7.

260. Stewart, P. E., R. Thalken, J. L. Bono, and P. Rosa. 2001. Isolation of a circular plasmid region sufficient for autonomous replication and transformation of infectious Borrelia burgdorferi. Mol Microbiol 39:714-21.

261. Stolp, H., and M. P. Starr. 1963. Bdellovibrio Bacteriovorus Gen. Et Sp. N., a Predatory, Ectoparasitic, and Bacteriolytic Microorganism. Antonie Van Leeuwenhoek 29:217-48.

262. Stricker, R. B., and E. E. Winger. 2003. Musical hallucinations in patients with Lyme disease. South Med J 96:711-5.

263. Suga, T., M. Hirano, M. Takayanagi, H. Koshimoto, and A. Watanabe. 1998. Restricted photorelease of biologically active molecules near the plasma membrane. Biochem Biophys Res Commun 253:423-30.

264. Surette, J. B. S. a. M. G. 1996. Chemotaxis, p. 1103-30. In F. C. Neidhardt (ed.), Escherichia coli and Salmonella Cellular and Molecular Biology, vol. 1. ASM Press, Wasington, DC.

265. Szczepanski, A., M. B. Furie, J. L. Benach, B. P. Lane, and H. B. Fleit. 1990. Interaction between Borrelia burgdorferi and endothelium in vitro. J Clin Invest 85:1637-47.

266. Taschner, S., A. Meinke, A. von Gabain, and A. P. Boyd. 2002. Selection of peptide entry motifs by bacterial surface display. Biochem J 367:393-402. 267. Telford, S. R., 3rd, T. N. Mather, S. I. Moore, M. L. Wilson, and A. Spielman. 1988. Incompetence of deer as reservoirs of the Lyme disease spirochete. Am J Trop Med Hyg 39:105-9.

268. Terekhova, D., M. L. Sartakova, G. P. Wormser, I. Schwartz, and F. C. Cabello. 2002. Erythromycin Resistance in Borrelia burgdorferi. Antimicrob Agents Chemother 46:3637-40. 
269. Thar, R., and M. Kuhl. 2003. Bacteria are not too small for spatial sensing of chemical gradients: An experimental evidence. Proc Natl Acad Sci U S A. 270. Thomas, J. C., M. Desrosiers, Y. St-Pierre, P. Lirette, J. G. Bisaillon, R. Beaudet, and R. Villemur. 1997. Quantitative flow cytometric detection of specific microorganisms in soil samples using rRNA targeted fluorescent probes and ethidium bromide. Cytometry 27:224-32.

271. Thomashow, L. S., and S. C. Rittenberg. 1985. Waveform analysis and structure of flagella and basal complexes from Bdellovibrio bacteriovorus 109J. J Bacteriol 163:1038-46.

272. Tilly, K., A. F. Elias, J. L. Bono, P. Stewart, and P. Rosa. 2000. DNA exchange and insertional inactivation in spirochetes. J Mol Microbiol Biotechnol 2:433-42.

273. Tilly, K., A. F. Elias, J. Errett, E. Fischer, R. Iyer, I. Schwartz, J. L. Bono, and P. Rosa. 2001. Genetics and regulation of chitobiose utilization in Borrelia burgdorferi. J Bacteriol 183:5544-53.

274. Tisa, L. S., and J. Adler. 1995. Cytoplasmic free-Ca2+ level rises with repellents and falls with attractants in Escherichia coli chemotaxis. Proc Natl Acad Sci U S A 92:10777-81.

275. Tourand, Y., K. Kobryn, and G. Chaconas. 2003. Sequence-specific recognition but position-dependent cleavage of two distinct telomeres by the Borrelia burgdorferi telomere resolvase, ResT. Mol Microbiol 48:901-11.

276. Trollmo, C., A. L. Meyer, A. C. Steere, D. A. Hafler, and B. T. Huber. 2001. Molecular mimicry in Lyme arthritis demonstrated at the single cell level: LFA-1 alpha $L$ is a partial agonist for outer surface protein A- reactive $T$ cells. J Immunol 166:5286-91.

277. Tso, W. W., and J. Adler. 1974. Negative chemotaxis in Escherichia coli. J Bacteriol 118:560-76.

278. Tsuda, M., and T. lino. 1983. Transductional analysis of the flagellar genes in Pseudomonas aeruginosa. J Bacteriol 153:1018-26.

279. Tsutsui, N., I. Taneike, T. Ohara, S. Goshi, S. Kojio, N. Iwakura, H. Matsumaru, N. Wakisaka-Saito, H. M. Zhang, and T. Yamamoto. 2000. A novel 
action of the proton pump inhibitor rabeprazole and its thioether derivative against the motility of Helicobacter pylori. Antimicrob Agents Chemother 44:3069-73.

280. Turner, L., W. S. Ryu, and H. C. Berg. 2000. Real-time imaging of fluorescent flagellar filaments. J Bacteriol 182:2793-801.

281. Umemoto, T., T. Jinno, Y. Taiji, and T. Ogawa. 2001. Chemotaxis of oral treponemes toward sera and albumin of rabbit. Microbiol Immunol 45:571-7. 282. Wadhams, G. H., A. C. Martin, S. L. Porter, J. R. Maddock, J. C. Mantotta, H. M. King, and J. P. Armitage. 2002. TlpC, a novel chemotaxis protein in Rhodobacter sphaeroides, localizes to a discrete region in the cytoplasm. Mol Microbiol 46:1211-21.

283. Wang, D., D. J. Botkin, and S. J. Norris. 2003. Characterization of the vls antigenic variation loci of the Lyme disease spirochaetes Borrelia garinii Ip90 and Borrelia afzelii ACAI. Mol Microbiol 47:1407-17.

284. Wang, G., A. P. van Dam, A. Le Fleche, D. Postic, O. Peter, G. Baranton, R. de Boer, L. Spanjaard, and J. Dankert. 1997. Genetic and phenotypic analysis of Borrelia valaisiana sp. nov. (Borrelia genomic groups VS116 and M19). Int J Syst Bacteriol 47:926-32.

285. Wang, G., A. P. van Dam, I. Schwartz, and J. Dankert. 1999. Molecular typing of Borrelia burgdorferi sensu lato: taxonomic, epidemiological, and clinical implications. Clin Microbiol Rev 12:633-53.

286. Ward, S. M., A. Delgado, R. P. Gunsalus, and M. D. Manson. 2002. A NarX-Tar chimera mediates repellent chemotaxis to nitrate and nitrite. Mol Microbiol 44:709-19.

287. Williams, C. J., Vandenberg, P., Prockop, D.J. 1997. Matrix Glycoproteins and Proteoglycans, p. 37-54. In W. N. Kelley (ed.), Textbook of Rheumatology, vol. 1. W.B. Saunders Company, Philadelphia.

288. Wilske, B., A. G. Barbour, S. Bergstrom, N. Burman, B. I. Restrepo, P. A. Rosa, T. Schwan, E. Soutschek, and R. Wallich. 1992. Antigenic variation and strain heterogeneity in Borrelia spp. Res Microbiol 143:583-96. 
289. Wilson, M. L., and A. Spielman. 1985. Seasonal activity of immature Ixodes dammini (Acari: Ixodidae). J Med Entomol 22:408-14.

290. Wilson, M. L. A., Gregory H., Spielman, Andrew. 1985. Correlation Between Abundance of Deer and That of the Deer Tick, Ixodes dammini (Acari: Ixodidae). Annals of the Entomological Society of American 78:172-176. 291. Wooten, R. M., Y. Ma, R. A. Yoder, J. P. Brown, J. H. Weis, J. F. Zachary, C. J. Kirschning, and J. J. Weis. 2002. Toll-like receptor 2 is required for innate, but not acquired, host defense to Borrelia burgdorferi. J Immunol 168:348-55. 292. Worku, M. L., R. L. Sidebotham, and Q. N. Karim. 1999. Effects of ranitidine bismuth citrate on Helicobacter pylori motility, morphology and survival. Aliment Pharmacol Ther 13:753-60.

293. Worku, M. L., R. L. Sidebotham, M. M. Walker, T. Keshavarz, and Q. N. Karim. 1999. The relationship between Helicobacter pylori motility, morphology and phase of growth: implications for gastric colonization and pathology. Microbiology 145:2803-11.

294. Yang, X. F., S. M. Alani, and M. V. Norgard. 2003. The response regulator Rrp2 is essential for the expression of major membrane lipoproteins in Borrelia burgdorferi. Proc Natl Acad Sci U S A 100:11001-6.

295. Yuri, K., Y. Takamoto, M. Okada, T. Hiramune, N. Kikuchi, and R. Yanagawa. 1993. Chemotaxis of Leptospires to hemoglobin in relation to virulence. Infect Immun 61:2270-2.

296. Zhang, J. R., J. M. Hardham, A. G. Barbour, and S. J. Norris. 1997. Antigenic variation in Lyme disease borreliae by promiscuous recombination of Vmp-like sequence cassettes. Cell 89:275-85.

297. Zhang, J. R., and S. J. Norris. 1998. Genetic variation of the Borrelia burgdorferi gene vlsE involves cassette-specific, segmental gene conversion. Infect Immun 66:3698-704.

298. Zhulin, I. B., and J. P. Armitage. 1993. Motility, chemokinesis, and methylation-independent chemotaxis in Azospirillum brasilense. J Bacteriol 175:952-8. 


\section{Curriculum Vitae: Richard Gerrit Bakker}

rbakker@hsc.wvu.edu

Richard Gerr
Ch4 Oakmo
Charleston,
Education


Presentations
and
Publications

Richard Gerrit Bakker 1234 Oakmont Road Charleston, West Virginia 25314 304.685 .3368
Richard Gerrit Bakker 3110 McCorkle Avenue, SE Charleston, West Virginia 25304 304.347.1319
2001: Entered Ph.D. candidacy

2000: Entered Dr. Nyles Charon's Laboratory

2000: Passed Step I of USMLE

1998 - Present: MD/Ph.D. Candidate at West Virginia University School of Medicine; Morgantown, West Virginia

1998: Cum Laude Graduate of Mount Union College; Alliance, Ohio: Bachelor of Science: Biology Major / Chemistry Minor

2004: Paper: Identification of specific chemoattractants for Borrelia burgdorferi: A FACS based chemotaxis assay. In preparation for Applied and Environmental Microbiology

2004: Poster: Chemically Defined Attractant for Borrelia burgdorferi: A FACS based approach. American Society for Microbiology General Meeting. June, 2004

2003: Poster: Chitobiose and N-acetyl-glucosamine are chemoattractants for Borrelia burgdorferi: A FACS based approach. Bacterial Locomotion and Signal Transduction Meeting, Cuernavaca, Mexico January 2003

2002: Paper: C. Li,, R. G. Bakker, M. A. Motaleb, M. L. Sartakova, F. C. Cabello, and N. W. Charon. 2002. Asymmetrical flagellar rotation in Borrelia burgdorferi nonchemotactic mutants. Proc Natl Acad Sci U S A 99:6169-74. April 2002

2001: Poster: Motion Analysis of Spirochetes Using a Computer Based Tracking System. American Society for Microbiology 
General Meeting. June, 2001

Professional Organizations and Positions

2003: West Virginia University Distinguished Teacher Committee 2000-2003: MD/PhD program student representative 2001-2002: Microbiology and Immunology graduate student representative

Community 2001 - present: Volunteer for West Virginia Public Radio Service 